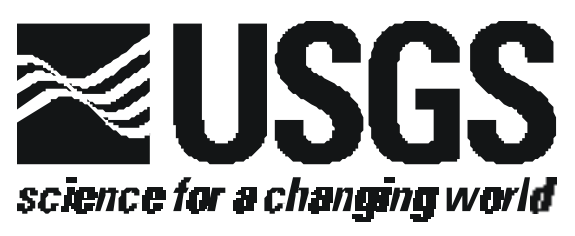

\title{
MAGNETOTELLURIC DATA ACROSS THE BATTLE MOUNTAIN-EUREKA AND CARLIN TRENDS, SOUTH OF CORTEZ, NEVADA
}

\author{
by \\ Jackie M. Williams, Brian D. Rodriguez, \\ and \\ Douglas P. Klein 1
}

Open-File Report 01-118

on-line edition

2001

This report is preliminary and has not been reviewed for conformity with U.S. Geological Survey editorial standards and stratigraphic nomenclature. Any use of trade, product, or firm names is for descriptive purposes only and does not imply endorsement by the U.S.

Government.

U.S. DEPARTMENT OF THE INTERIOR

U.S. GEOLOGICAL SURVEY

${ }^{1}$ Denver, Colorado 
TABLE OF CONTENTS

INTRODUCTION

MAGNETOTELLURIC METHOD 3

MAGNETOTELLURIC SURVEY

MAGNETOTELLURIC DATA $\quad 6$

REFERENCES CITED 9

APPENDIX - MAGNETOTELLURIC DATA PLOTS 12 


\section{INTRODUCTION}

Genesis of gold deposits along the Battle Mountain-Eureka and Carlin trends in northern Nevada is not fully understood and subject to conflicting models (e.g. Arehart and others, 1993; Ilchik and Barton, 1997; Radtke, 1985; Shawe, 1991; Sillitoe and Bonham, 1990; Tosdal, 1998). A general consensus among these models is that regional structures somehow controlled the spatial distribution of the deposits. To investigate crustal structures that may be related to the genesis of gold deposits along these trends, a regional southwest-northeast profile of magnetotelluric (MT) soundings was acquired in 1994, 1996, 1997, and 2000 (line MT2-MT2", Figure 1). Resistivity modeling of the MT data can be used to infer the deep resistivity structure of the crust to help investigate possible tectonic controls on the emplacement of mineral deposits along these linear trends.

\section{MAGNETOTELIURIC METHOD}

The magnetotelluric (MT) method is a passive surface geophysical technique, which uses the earth's natural electromagnetic fields to investigate the electrical resistivity structure of the subsurface. The resistivity of geologic units is largely dependent upon their fluid content, porosity, fracturing, temperature, and conductive mineral content (Keller, 1989). Saline fluids within the pore spaces and fracture openings can reduce resistivities in a resistive rock matrix. Also, resistivity can be lowered by the presence of conductive clay minerals, carbon, and metallic

mineralization. It is common for altered volcanic rocks to contain authigenic minerals that have resistivities ten times lower than those of the surrounding rocks (Nelson and Anderson, 1992). Increased temperatures cause higher ionic mobility and mineral activation energy, reducing rock resistivities significantly. Unaltered, unfractured igneous rocks are normally very resistive (typically 1,000 ohm-m or greater), whereas faults will show low resistivity (less than $100 \mathrm{ohm}-\mathrm{m}$ ) when they are comprised of rocks fractured enough to have hosted fluid transport and consequent mineralogical alteration (Eberhart-Phillips et al., 1995). Carbonate rocks are moderately to highly resistive (hundreds to thousands of ohm-m) dependent upon their fluid content, porosity, fracturing, and impurities. Marine shales, mudstones, and clay-rich alluvium are normally very conductive (a few ohm-m to tens of ohm-m). Unaltered, metamorphic rocks (nongraphitic) are moderately to highly resistive (hundreds to thousands of ohm-m). Tables of electrical resistivity for a 
variety of rocks, minerals and geological environments may be found in Keller (1987) and Palacky (1987).

The MT method allows us to probe the crust from depths of tens of meters to depths of tens of kilometers (Vozoff, 1991). Natural variations of the Earth's magnetic and electric field are measured and recorded at each MT station. Frequencies of $10,000 \mathrm{~Hz}$ to $1 \mathrm{~Hz}$ from worldwide lightning activity and $1 \mathrm{~Hz}$ to $0.0001 \mathrm{~Hz}$ from geomagnetic micro-pulsations are the main frequency bands used by the MT method. The natural electric and magnetic fields propagate vertically in the earth because the very large resistivity contrast between the air and the earth causes a vertical refraction of both fields transmitted into the earth (Vozoff, 1972).

The natural electric and magnetic fields are recorded in two orthogonal, horizontal directions. The vertical magnetic field ("tipper") is also recorded. The resulting time-series signals are used to derive earth tensor apparent resistivities and phases by first converting them to complex cross-spectra using FFT (fast-Fourier-transform) techniques. Least-squares, cross-spectral analysis (Bendat and Piersol, 1971) is used to solve for a tensor-transfer function that relates the observed electric fields to the magnetic fields under the assumption that the Earth consists of a two-input, two-output, linear system with the magnetic fields as input and the electric fields as output (Rodriguez and others, 1996). Prior to conversion to apparent resistivity and phase, the tensor is normally rotated into principal directions that usually correspond to the direction of maximum and minimum apparent resistivity. For a two-dimensional (2-D) Earth, the MT fields can be de-coupled into transverse electric (TE) and transverse magnetic (TM) modes; 2-D modeling is generally done to fit both modes. When the geology satisfies the 2-D assumption, the MT data for the TE mode is assumed to represent the situation when the electric field is along the geologic strike, and the data for the TM mode is assumed to represent the situation when the electric field is across strike. The MT method is well suited for studying complicated geological environments because the electric and magnetic relations are sensitive to vertical and horizontal variations in resistivity. The method is capable of establishing whether the electromagnetic fields are responding to subsurface terranes of effectively 1-, 2-, or 3-dimensions. An introduction to the $\mathrm{MT}$ method and references for a more advanced understanding are contained in Dobrin and Savit (1988) and Vozoff (1991). 


\section{MAGNETOTELLURIC SURVEY}

Thirteen MT soundings were located along or near profile MT2-MT2" (Figure 1) with spacing that varied from 6 to 19.5 kilometers. The profile orientation is roughly perpendicular to the Battle Mountain-Eureka and Carlin trends. Stations 610, 17, and 18 were collected with our U.S. Geological Survey truck-mounted MT system (Stanley, 1978). Stations 11, 36, 107, and 110-112 were collected with our portable EMI MT-1 system (EMI, 1996). Horizontal electric fields were sensed using an L-shaped, three-electrode array with dipole lengths of $30 \mathrm{~m}$ except for stations $6-10,17$, and 18 where a dipole length of $37.5 \mathrm{~m}$ was used with our truck-mounted MT system. The orthogonal, horizontal magnetic fields in the direction of the electric-field measurement array were sensed using permalloy-cored induction coils (Stanley and Tinkler, 1983). Frequencies sampled ranged from 300 to $0.004 \mathrm{~Hz}$ using single station recordings of both orthogonal horizontal components of the electric and magnetic fields, along with the vertical magnetic field at stations 11, 36, 107, and 110-112. Sampling this frequency range in previous areas of widely varying geology has allowed us to probe the crust from depths of hundreds of meters to depths of tens of kilometers.

The recorded time-series data were transformed to the frequency domain and Fourier analyzed to determine a twodimensional apparent resistivity and phase tensor at each site. The data were rotated to maximum and minimum apparent resistivity directions so that propagation modes for the signals were decoupled into TE and TM modes. Local reference sensors to help reduce bias in the impedance determinations due to instrument or environmental noise (Gamble and others, 1979a; Clarke and others, 1983) were only used at station 36. Although true remote reference techniques were not used in our survey, we did sort cross-power files to select optimal signal-to-noise data sets (Appendix).

The effects of near-surface resistivity anomalies cause "static shifts" (Sternberg and others, 1988) in the data. Static shifts of this data set ranged from 0.0 to 0.3 of a log decade. Only stations 6,7 and 110 had static shifts of 0.3 of a log decade. The remainder of the stations had an average of 0.1 of a $\log$ decade static shift. 


\section{MAGNETOTELIURIC DATA}

The following table shows thirteen magnetotelluric (MT) station locations (from southwest to northeast). Coordinates are referenced to the 1866 Clarke spheroid and North American 1927 Western United States datum. Longitude and latitude format below is decimal degrees. Elevation is in meters.

\begin{tabular}{|c|c|c|c|}
\hline Station & Longitude & Latitude & $\operatorname{Elev}(\mathrm{m})$ \\
\hline$\overline{107}$ & $\overline{-116.92058}$ & $\overline{39.91737}$ & 1740 \\
\hline 17 & -116.83356 & 39.96701 & 1950 \\
\hline 11 & -116.73088 & 39.94657 & 1770 \\
\hline 10 & -116.65782 & 40.03011 & 1790 \\
\hline 6 & -116.58502 & 40.03230 & 1720 \\
\hline 9 & -116.48118 & 40.06390 & 1790 \\
\hline 8 & -116.42011 & 40.08985 & 1725 \\
\hline 7 & -116.32249 & 40.09119 & 1725 \\
\hline 18 & -116.16121 & 40.09325 & 1840 \\
\hline 111 & -116.00637 & 40.04967 & 1765 \\
\hline 110 & -115.85010 & 40.11423 & 1780 \\
\hline 112 & -115.73823 & 40.17175 & 1740 \\
\hline 36 & -116.13596 & 40.26943 & 1610 \\
\hline
\end{tabular}

The figures in the Appendix represent the raw field MT data for each station after the time series data was converted to the frequency domain and least-squares, cross-spectral analysis (Bendat and Piersol, 1971) was used to solve for a tensor-transfer function. This tensor transfer function was used to rotate the tensor into principal directions that correspond to the direction (rotation angle) of maximum and minimum apparent resistivity. Apparent resistivity and impedance phase were then calculated.

For each station, nine separate graph plots are given:

- Apparent Resistivity for two modes,

- Impedance Phase for two modes,

- Rotation Angle for the impedance tensor,

- Impedance Skew for the impedance tensor,

- Multiple Coherency for two modes of the electric field,

- Impedance Polar Plots (at 12 selected frequencies),

- Tipper Magnitude for the vertical magnetic field,

- Tipper Strike for the vertical magnetic field, and

- HzHx (x symbol) and HzHy (o symbol) Coherency. 
Each of these graphs plots the above quantity versus frequency. The Apparent Resistivity, Impedance Phase, and Multiple Coherency plots use $x$ and 0 symbols to distinguish the two tensor modes. When inverting or modeling the data, these modes will be identified as TE and TM. The $x$ and 0 symbols on the $\mathrm{HzHx}$ and $\mathrm{HzHy}$ Coherency plot represent $\mathrm{HzHx}$ and HzHy coherency, respectively. Error bars are probable errors within one standard deviation of the sample variance (Gamble and others, 1979b).

Apparent resistivity is a measure of the magnitude of the electric field strength over the magnetic field strength for a given frequency. The impedance phase is proportional to the slope of the apparent resistivity curve on a log-log scale, but from a baseline at -45 degrees (Vozoff, 1991). A measure of the dimensionality for MT data is provided by the impedance skew of the impedance tensor (Vozoff, 1972). If the effective measured resistivity response to the geology beneath a MT station is truly $1-D$ or $2-D$, then the skew will be zero. Both instrument and environmental sources of noise contribute to non-zero skew values, but are typically small (about 0.1) for relatively low noise level recordings. Higher skews (above 0.2) are an indication of either 3-D resistivity responses to the geology or higher levels of noise. Man-made electrical noise, such as power lines, power generators, moving vehicles and trains can have a negative effect on MT data quality. All these local disturbances produce an incoherent noise mainly affecting higher frequencies, usually above $1 \mathrm{~Hz}$. Other man-made electrical noise, such as direct current electric trains and active cathodic protection of pipelines produce coherent electromagnetic signals mainly affecting frequencies below $1 \mathrm{~Hz}$.

In the survey area, noise from a number of small power lines and small moving vehicles was negligible at distances of $0.4 \mathrm{~km}$ and greater. Power line levels were measured at each site and were typically less than $20 \%$ of the maximum recordable signals. Noise from larger power lines, power generators, pipelines, railroads, and steam-driven trains, mostly near mining operations, was negligible at least $5 \mathrm{~km}$ from them. Recordings were not made when noise from moving vehicles affected the magnetic signals. Local lightning, wind, and rainstorms can also degrade data quality, but these were avoided by not recording during active thunderstorm periods. Wind noise was minimized by burying the magnetic induction coils. 
Predicted values of the electric field can be computed from the measured values of the magnetic field (Vozoff, 1991). The coherence of the predicted electric field with the measured electric field is a measure of the signal-to-noise ratio provided in the multiple coherency plot. Values are normalized between 0 and 1 , where values at 0.5 signify signal levels equal to noise levels. For this data set, coherencies were generally at an acceptable level, except at times in the "dead band" (0.1 to $1 \mathrm{~Hz})$ and at times in the lower frequencies $(0.004$ to 0.1$)$. The lower frequency ionospheric signals are related to sunspot activity whose levels typically follow an 11-year cycle. The sunspot activity was near the lowest level of the cycle during the 1994, 1996, and 1997 surveys, and was near the highest level in the 2000 survey.

The figures in the Appendix represent the raw field MT data at each station, which includes some data scatter and poor signal-to-noise ratios. Our only effort at removing noisy data points was to visually inspect and select the best signal-to-noise field data to combine into the final data plots.

The impedance polar plots provide a measure of the MT data dimensionality (Reddy and others, 1977). For 1-D resistivity structures, the principal impedance polar diagrams are circles. For 2-D or 3-D resistivity structures, the principal impedance polar diagrams (dashed lines) elongate either parallel or perpendicular to strike direction. Over resistors, the principal impedance polar diagrams elongate perpendicular to strike direction and over conductors, the principal impedance polar diagrams elongate parallel to strike direction. Also, for 2-D resistivity structures, the additional impedance polar diagrams (solid lines) attain the shape of a symmetric clover leaf. For 3-D resistivity structures, the additional impedance polar diagrams elongate in one direction and their amplitudes are comparable to those of principal impedances. Sites whose polar plots indicated 3D character in the lower frequencies were MT stations 6, 8-11, 17, 18, 36, 110, and 112 (Figure 1).

The tipper can be solved for when the vertical component of the magnetic field is measured. The tipper magnitude is a measure of the tipping of the magnetic field out of the horizontal plane (Vozoff, 1991). The magnitude is zero for the $1-\mathrm{D}$ case and typically increases between 0.1 to 0.5 , and rarely as great as 1 , as it responds to vertical and subvertical structures. The tipper strike is typically used to help resolve the 90-degree ambiguity in the impedance rotation 
angle. The vertical component of the magnetic field was measured at MT stations 11, 36, 107, and 110-112. The tipper magnitude of these stations was typically 0.1 to 0.4 over the lower frequencies indicating vertical structure at depth. The $\mathrm{HzHx}$ and HzHy coherency is a measure of the signal-to-noise ratio of the vertical magnetic field with respect to each of the orthogonal horizontal magnetic field directions. Values are normalized between 0 and 1 , where values at 0.5 signify signal levels equal to noise levels. These three-component magnetic field coherencies provide a check on the signal-tonoise ratio of the measured values in the tipper magnitude and tipper strike plots.

\section{REFERENCES CITED}

Arehart, G.B., Foland, K.A., Naeser, C.W., and Kesler, S.E., 1993, 40Ar/39Ar, K/Ar, and fission track geochronology of sediment-hosted disseminated gold deposits at post-Betze, Carlin Trend, northeastern Nevada: Economic Geology, vol. 88 , no. 3, p. $622-646$.

Bendat, J.S., and Piersol, A.G., 1971, Random Data: Analysis and Measurement Procedures: New York, Wiley Interscience, $407 \mathrm{p}$.

Clarke, J., Gamble, T.D., Goubau, W.M., Koch, R.H., and Miracky, R.F., 1983, Remote-reference magnetotellurics: Equipment and procedures: Geophysical Prospecting, vol. 31 , p. 149-170.

Dobrin, M.D., and Savit, C.H., 1988, Introduction to Geophysical Prospecting (4th ed.): New York, McGraw-Hill, $867 \mathrm{p}$.

Eberhart-Phillips, D., Stanley, W. D., Rodriguez, B. D. and Lutter, W. J., 1995, Surface seismic and electrical methods to detect fluids related to faulting: Journal of Geophysical Research, vol. 100, no. B7, pp. 12,91912,936 .

EMI, 1996, MT-1 magnetotelluric system operation manual, version 3.2: ElectroMagnetic Instruments, Inc., Richmond, California, $220 \mathrm{p}$.

Gamble, T.D., Goubau, W.M. and Clarke, J., 1979a, Magnetotellurics with a remote magnetic reference: Geophysics, v. 44, no. 1, p. 53-68. 
Gamble, T.D., Goubau, W.M. and Clarke, J., 1979b, Error analysis for remote reference magnetotellurics: Geophysics, v. 44, no. 5, p. 959-968.

Ilchik, R.P. and Barton, M.D., 1997, An amagmatic origin of Carlin-type gold deposits: Economic Geology, vol. 92, no. 3 , p. 269-288.

Keller, G.V., 1987, Rock and mineral properties, in Electromagnetic Methods in Applied Geophysics Theory: M.N. Nabighian, Ed., Society of Exploration Geophysicists, Tulsa, Oklahoma, v. 1, p. 1351.

Keller, G.V., 1989, Electrical properties, in Carmichael, R.S., Ed., Practical handbook of physical properties of rocks and minerals: CRC Press, Boca Raton, Florida, p. $359-427$.

Nelson, P.H. and Anderson, L.A., 1992, Physical properties of ash flow tuff from Yucca Mountain, Nevada: Journal of Geophysical Research, vol. 97, no. B5, p. 6823-6841.

Palacky, G.J., 1987, Resistivity characteristics of geologic targets, in Electromagnetic Methods in Applied Geophysics Theory: M.N. Nabighian, Ed., Society of Exploration Geophysicists, Tulsa, Oklahoma, vol. 1, p. 53129.

Radtke, A.S., 1985, Geology of the Carlin gold deposit, Nevada: U.S. Geological Survey Professional Paper 1267, $124 \mathrm{p}$.

Reddy, I.K., Rankin, D., and Phillips, R.J., 1977, Threedimensional modeliing in magnetotelluric and magnetic variational sounding: Geophysics Journal of the Royal Astronomical society, vol. 51, p. 313-325.

Rodriguez, B.D., Stanley, W.D., and Williams, J.M., 1996, Axial structures within the Reelfoot rift delineated with magnetotelluric surveys: U.S. Geological Survey Professional Paper 1538-K, $30 \mathrm{p}$.

Shawe, D.R., 1991, Structurally controlled gold trends imply large gold resources in Nevada, in Geology and ore deposits of the Great Basin, Symposium Proceedings: Raines, G.L., Lisle, R.E., Schafe, R.W., Wilkinson, W.H., Eds., Geological Society of Nevada, Reno, vol. 1, p. 199212 . 
Sillitoe, R.H. and Bonham, H.F., 1990, Sediment-hosted gold deposits; distal products of magmatic-hydrothermal systems: Geology, vol. 18, no. 2, p. 157-161.

Stanley, W.D., 1978, New magnetotelluric field processing system: U.S. Geological Survey Professional Paper 1100 , $174 \mathrm{p}$.

Stanley, W.D., and Tinkler, R.D., 1983, A practical, low-noise coil system for magnetotellurics: U.S. Geological Survey Open-File Report 83-85, $18 \mathrm{p}$.

Sternberg, B.K., Washburne, J.C., and Pellerin, L., 1988, Correction for the static shift in magnetotellurics using transient electromagnetic soundings: Geophysics, vol. 53, p. 1459-1468.

Struhsacker, E.M., Jones, E., and Green, S.M., 1996, Roadside geology and precious-metal mineralization along the I-80 corridor, Reno to Elko, Nevada, in Struhsacker, E.M. and Green, S.M., eds., Geology and ore deposits of the American Cordillera - Field Trip Guidebook Compendium: Geological Society of Nevada, Reno, Nevada, p. 3.

Tosdal, R.M., 1998, Contributions to the gold metallogeny of northern Nevada: U.S. Geological Survey Open-File Report $98-338,290 \mathrm{p}$.

Vozoff, K., 1972, The magnetotelluric method in the exploration of sedimentary basins: Geophysics, vol. 37, p. $98-141$.

Vozoff, K., 1991, The magnetotelluric method, in Electromagnetic methods in applied geophysics: M.N. Nabighian, Ed., Society of Exploration Geophysicists, Tulsa, Oklahoma, vol. 2, part B, p. 641-711. 


\section{APPENDIX}

\section{MAGNETOTELLURIC DATA PLOTS}

For stations 11, 36, 107, and 110-112, there are nine separate graph plots:

- Apparent Resistivity for two modes,

- Impedance Phase for two modes,

- Rotation Angle for the impedance tensor,

- Impedance Skew for the impedance tensor,

- Multiple Coherency for two modes of the electric field,

- Impedance Polar Plots (at 12 selected frequencies),

- Tipper Magnitude for the vertical magnetic field,

- Tipper strike for the vertical magnetic field, and

- HzHx (x symbol) and HzHy (o symbol) Coherency.

Stations 6-10, 17, and 18, only have the first six graph plots above, since the vertical magnetic field data (Tipper, $\mathrm{Hz}$ ) was not acquired. Refer to the "Magnetotelluric Data" section in this report for an explanation of these plots. 


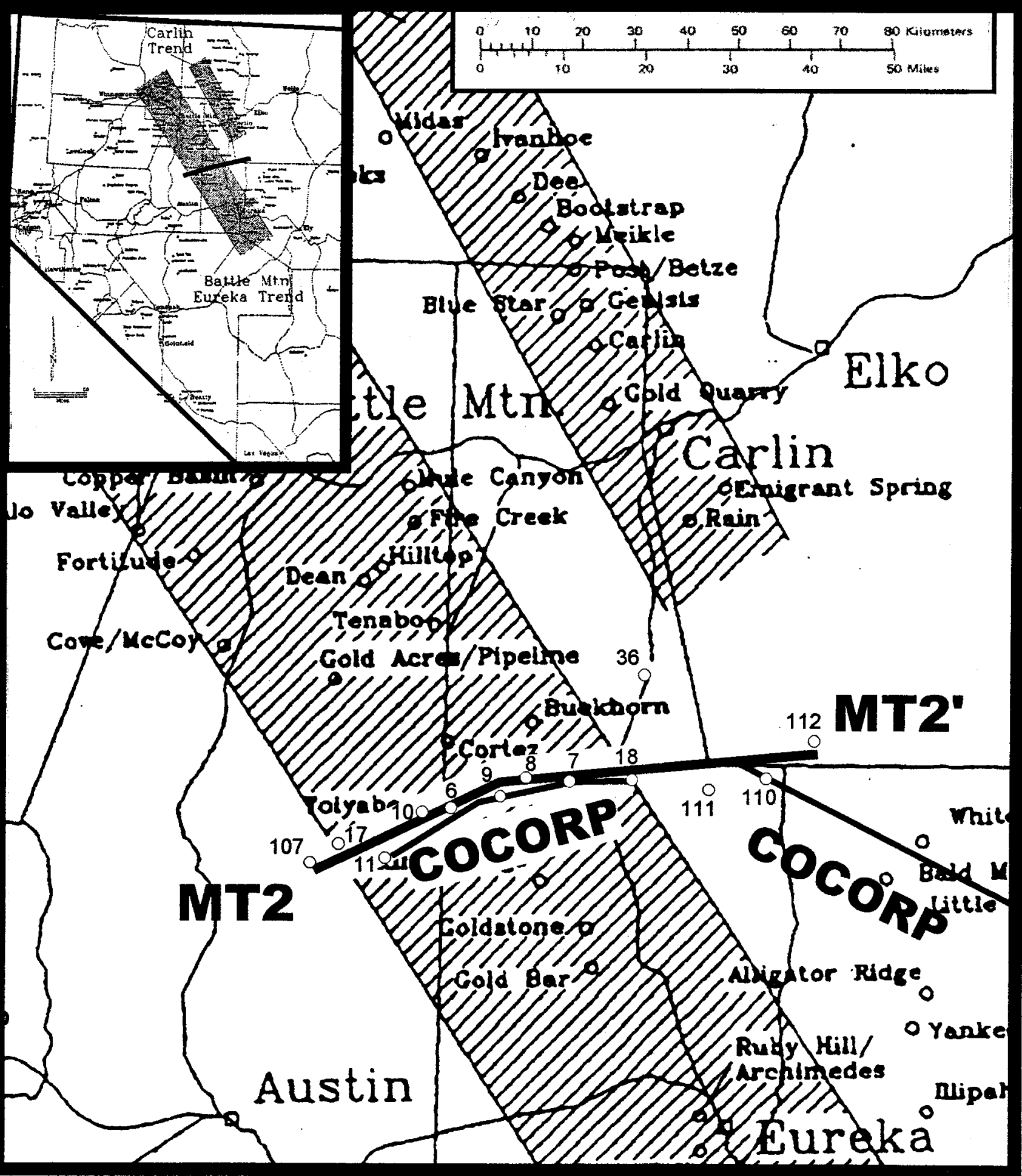

Figure 1. Index map. Magnetotelluric transect (MT2-MT2') acquired in 1994. 1996, 1997, and 2000 in northeastern Nevada. Shaded zones are two northwesttrending mineralized belts in northeastern Nevada, the well-known Carlin trend and the Battle Mountain-Eureka trend. Base map adapted from struhsacker and others (1996). 
Station 107

APPARENT RESISTIVITY Simpson park Mts,NV 100k

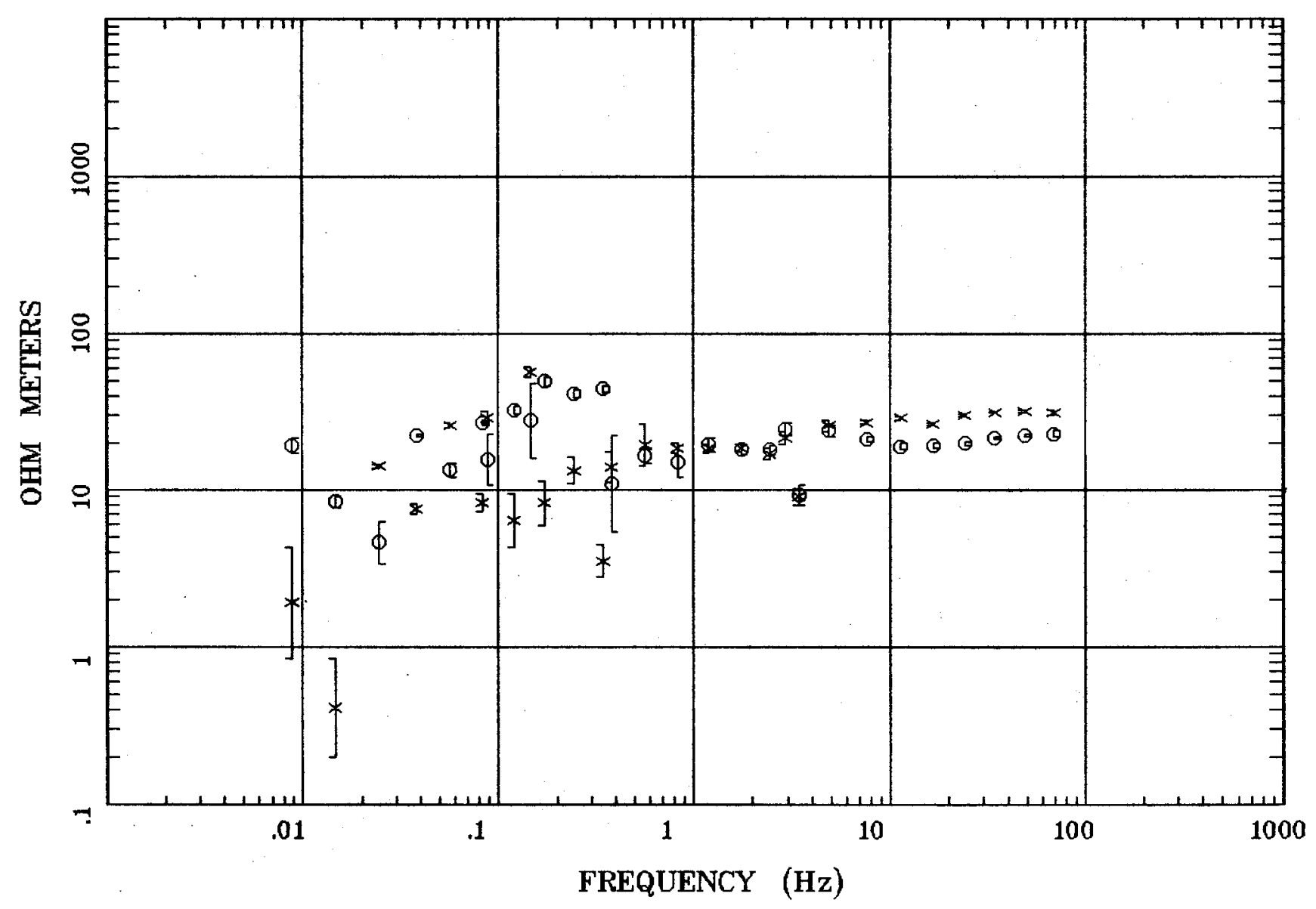

Client:

Remote: none

Acquired: 14:2 Aug 05, 2000

Survey Co:USGS
Rotation:

Filename: hr107c.avg

Channels: Ch1 Ch2 Ch3 Ch4 Ch5 Ch3 Ch4

Plotted: 09:41 Jan 24, 2001

< EMI - ElectroMagnetic Instruments > 


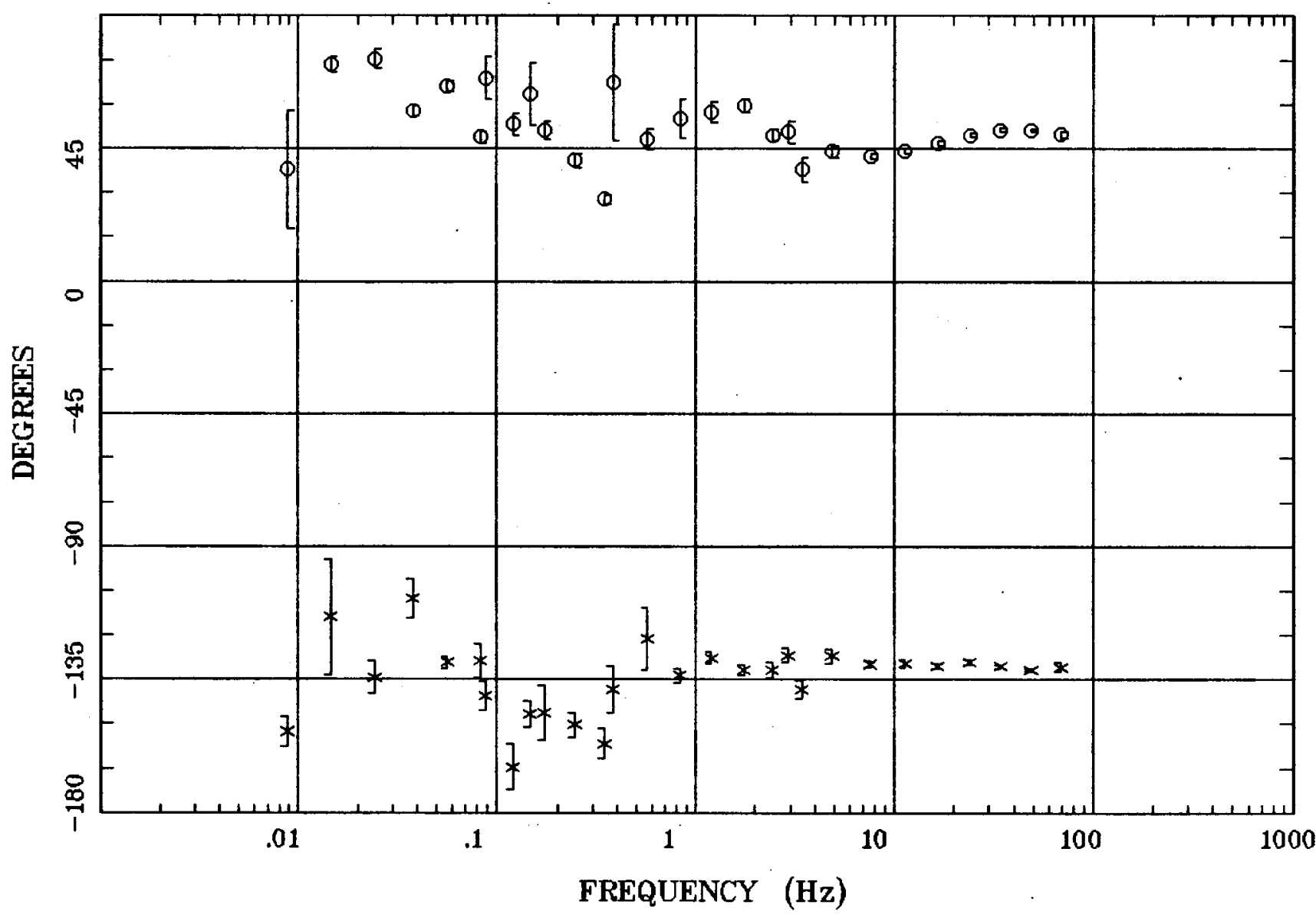

Client:

Remote: none

Acquired: 14:2 Aug 05, 2000

Survey Co:USGS
Rotation:

Filename: hr107c.avg

Channels: Ch1 Ch2 Ch3 Ch4 Ch5 Ch3 Ch4 Plotted: 09:41 Jan 24, 2001

< EMI - ElectroMagnetic Instruments > 


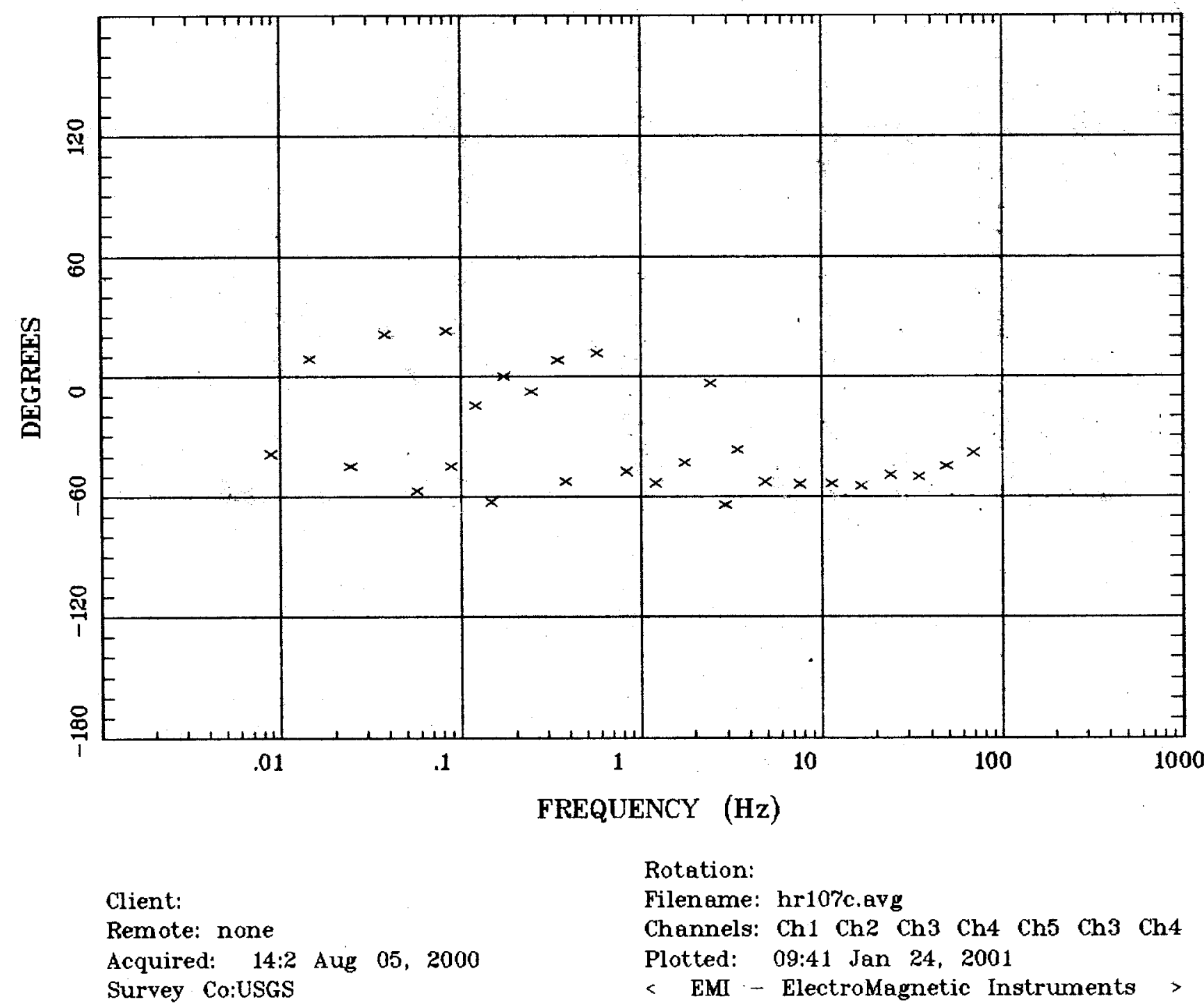


Station 107

IMPEDANCE SKEW

Simpson park Mts,NV 100k

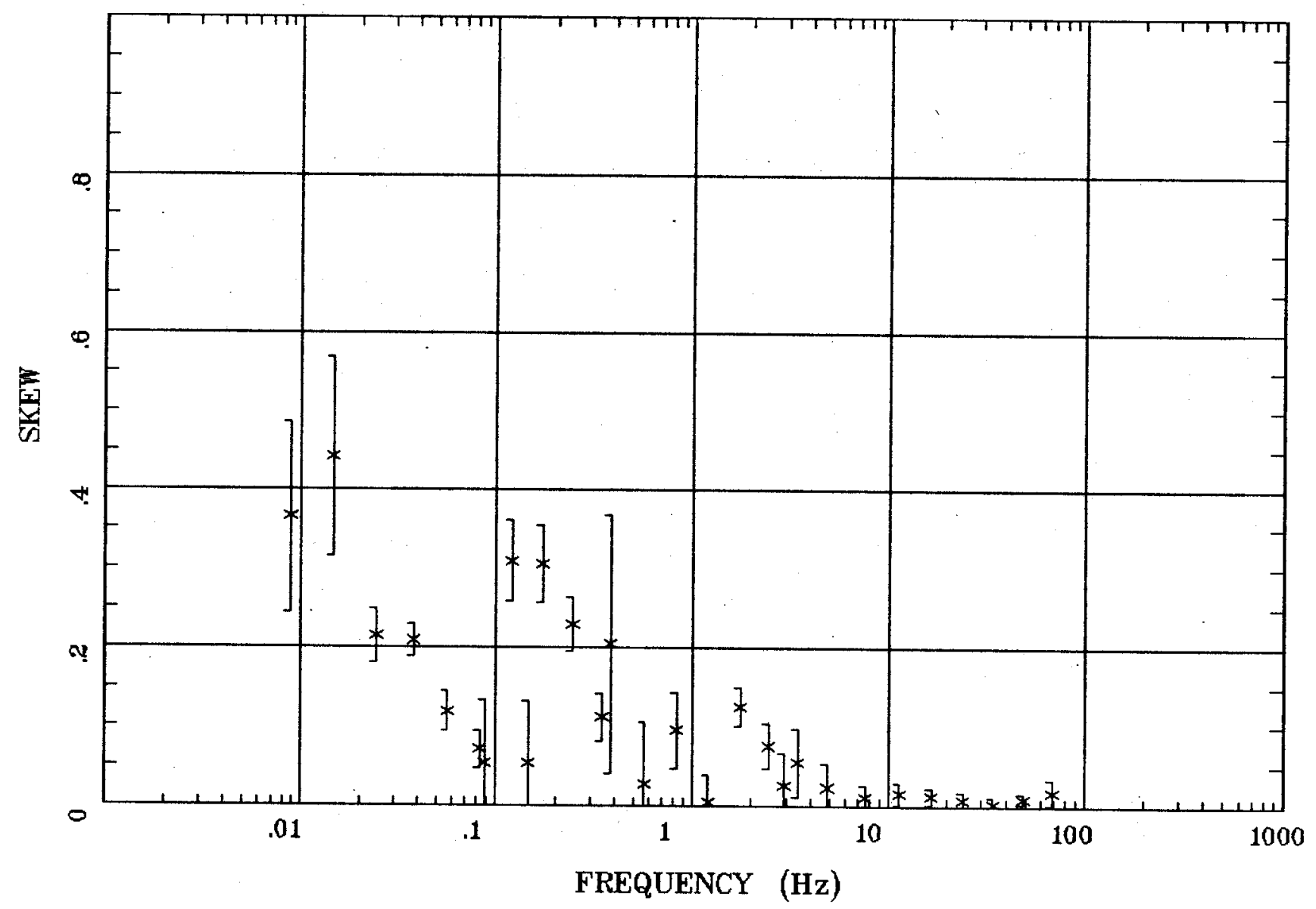

Client:

Remote: none

Acquired: 14:2 Aug 05, 2000

Survey Co:USGS
Rotation:

Filename: hr107c.avg

Channels: Ch1 Ch2 Ch3 Ch4 Ch5 Ch3 Ch4

Plotted: 09:41 Jan 24, 2001

$<$ EMI - ElectroMagnetic Instruments > 
E MULT Coh.

Simpson park Mts,NV 100k

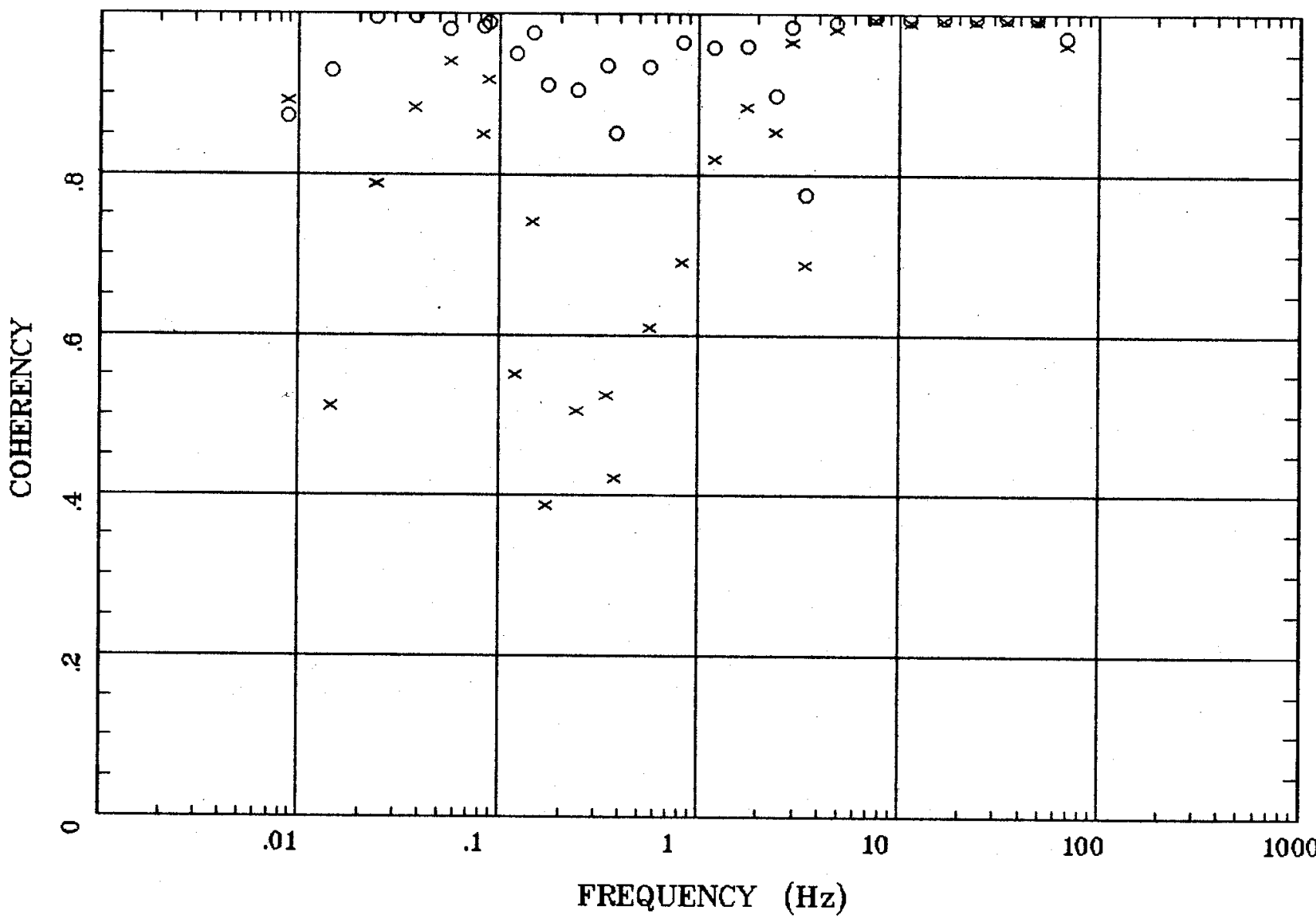

Client:

Remate: none

Acquired: 14:2 Aug 05, 2000

Survey Co:USGS
Rotation:

Filename: hr107c.avg

Channels: Ch1 Ch2 Ch3 Ch4 Ch5 Ch3 Ch4

Plotted: 09:41 Jan 24, 2001

$<$ EMI - ElectroMagnetic Instruments 


\section{Station 107}

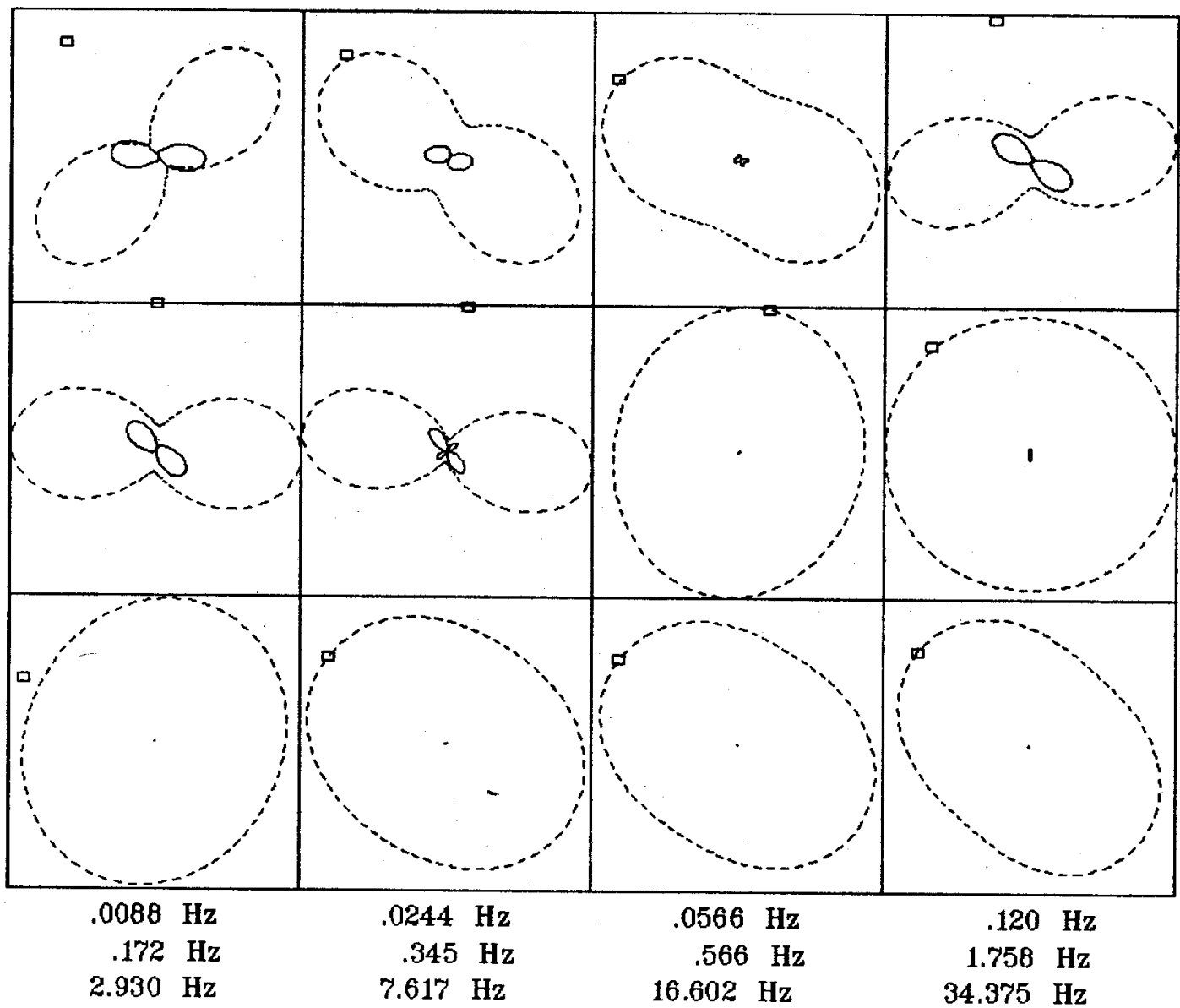

Client:

Rotation:

Remote: none

Acquired: 14:2 Aug 05, 2000

Filename: hr107c.avg

Channels: Ch1 Ch2 Ch3 Ch4 Ch5 Ch3 Ch4

Plotted: 09:41 Jan 24, 2001

Survey Co:USGS

< EMI - ElectroMagnetic Instruments 
Station 107

TIPPER MAGNITUDE

Simpson park Mts,NV 100k

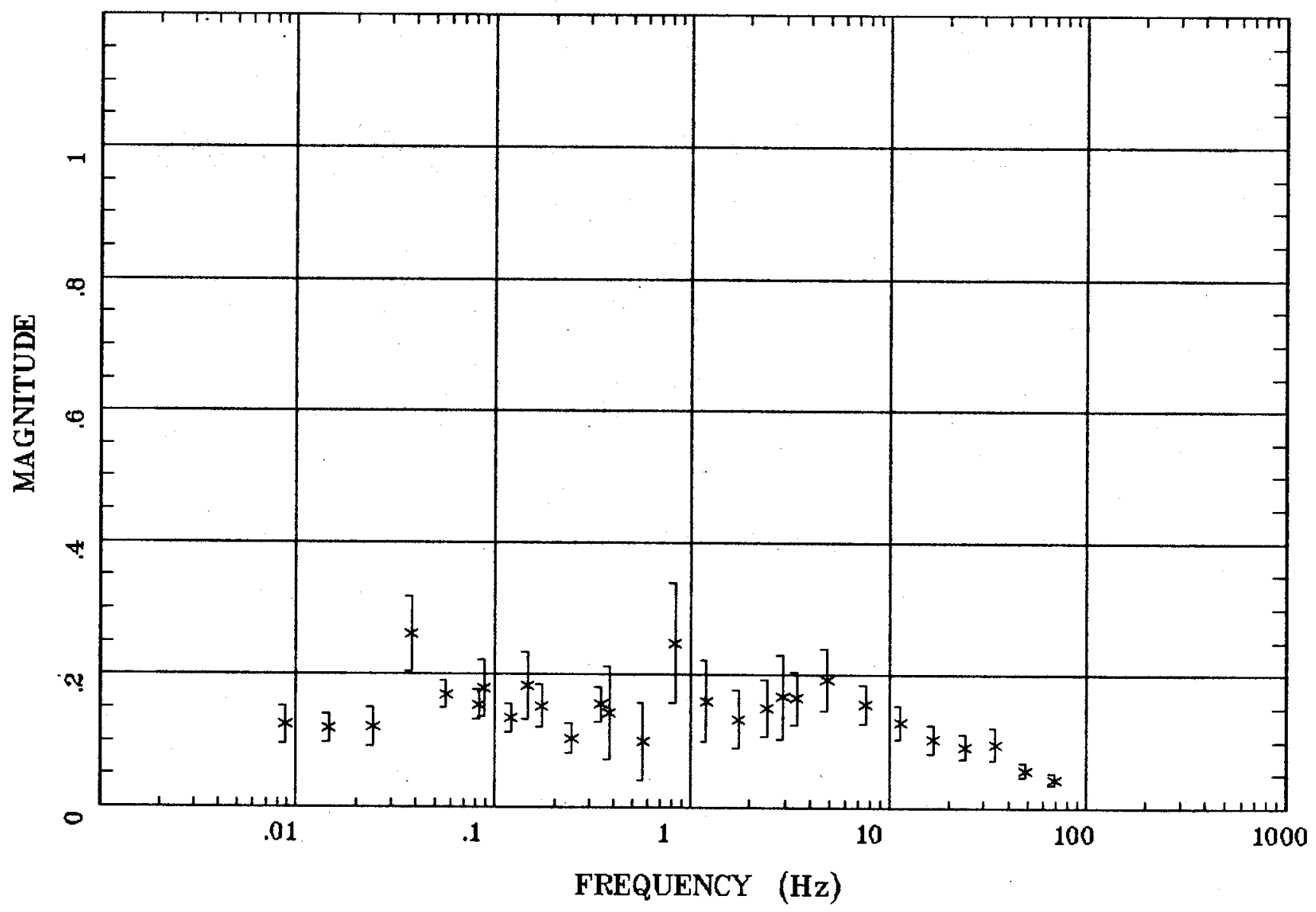

Client:

Remote: none

Acquired: 14:2 Aug 05, 2000 Survey Co:USGS

\section{Rotation:}

Filename: hr107c.avg

Channels: Ch1 Ch2 Ch3 Ch4 Ch5 Ch3 Ch4 Plotted: 09:41 Jan 24, 2001

$<$ EMI - ElectroMagnetic Instruments 


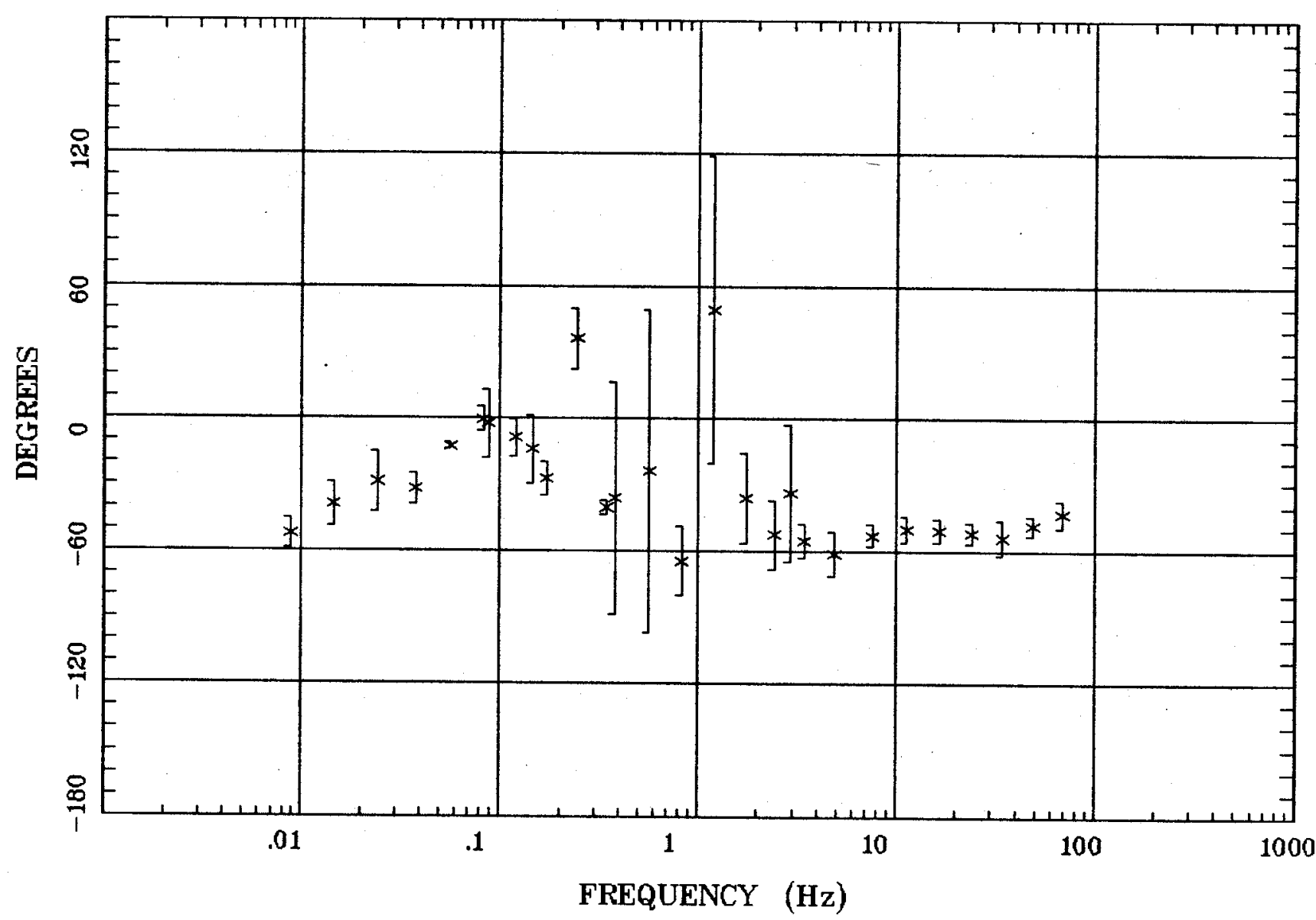

Client:

Remote: none Acquired: 14:2 Aug 05, 2000 Survey Co:USGS
Rotation:

Filename: hr107c.avg

Channels: Ch1 Ch2 Ch3 Ch4 Ch5 Ch3 Ch4 Plotted: 09:41 Jan 24, 2001

< EMI - ElectroMagnetic Instruments 
HzHx.x Coh HzHy.o

Simpson park Mts,NV 100k

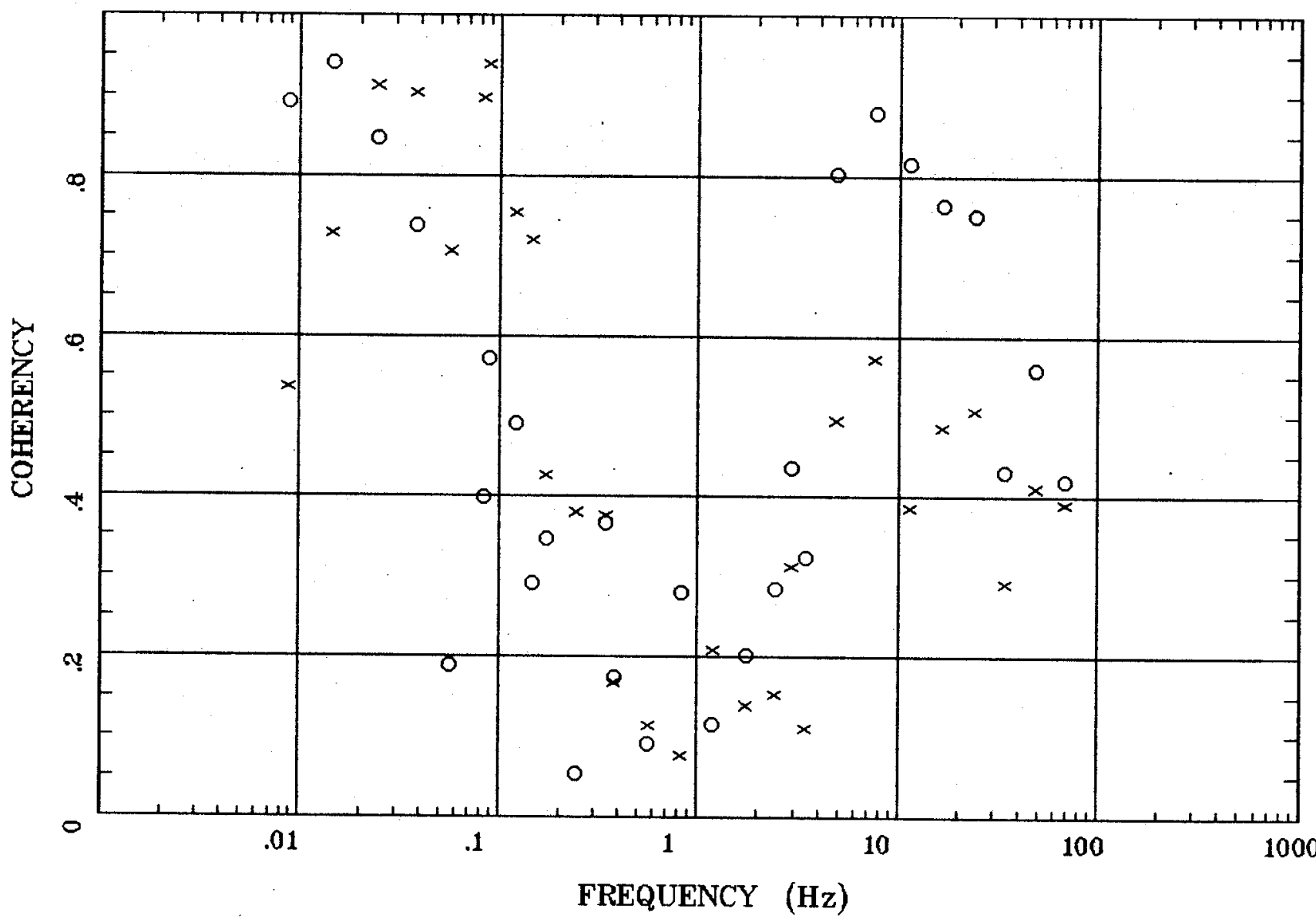

Client:

Remote: none

Acquired: 14:2 Aug 05, 2000 Survey Co:USGS
Rotation:

Filename: hr107c.avg

Channels: Ch1 Ch2 Ch3 Ch4 Ch5 Ch3 Ch4

Plotted: 09:41 Jan 24, 2001

$<$ EMI - ElectroMagnetic Instruments 
APPARENT RESISTIVITY

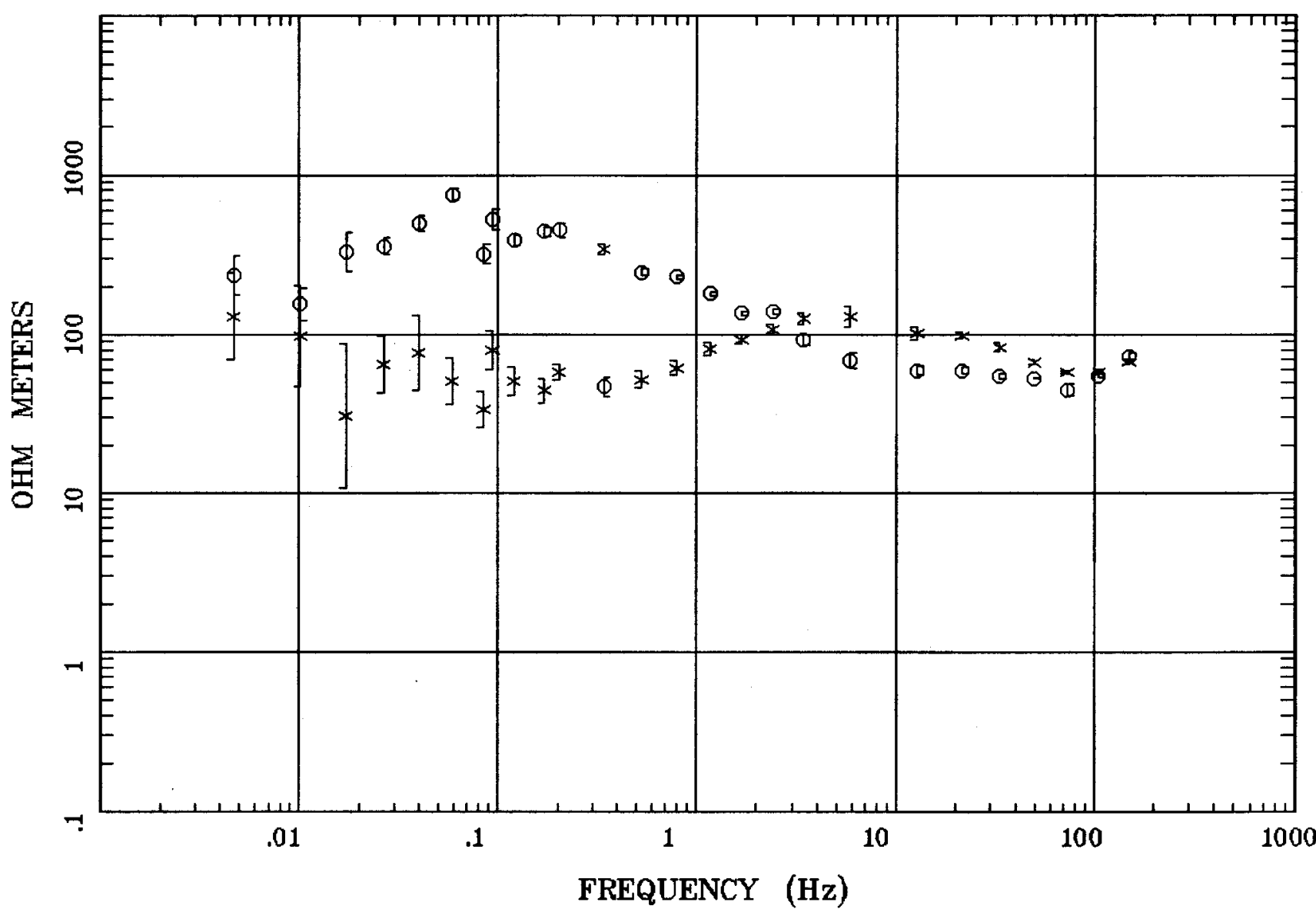

Client:

Remote:

Acquired:

Survey Co:
Rotation:

Filename: CT17

Channels: Ch1 Ch2 Ch3 ch4 Ch5 Ch6 Chr

Plotted: 10:15 Jun 25, 1999

$<$ EMI - ElectroMagnetic Instruments 


\section{Station 17}

\section{IMPEDANCE PHASE}

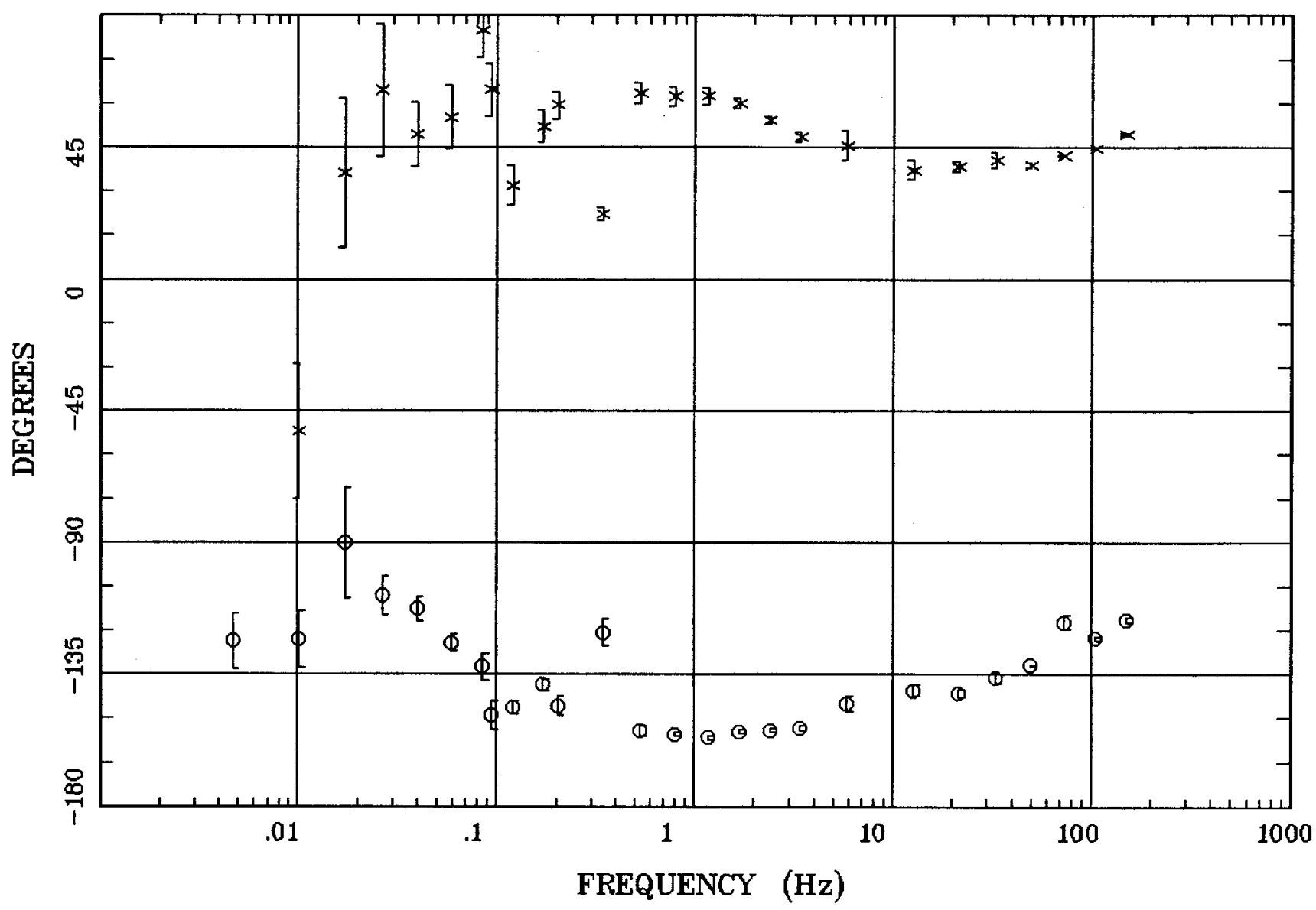

Client:

Rotation:

Remote:

Filename: CT17

Channels: Ch1 Ch2 Ch3 Ch4 Ch5 Ch6 Ch7

Acquired:

Plotted: 10:15 Jun 25, 1999

Survey Co:

< EMI - ElectroMagnetic Instruments 


\section{Station 17}

\section{ROTATION ANGLE}

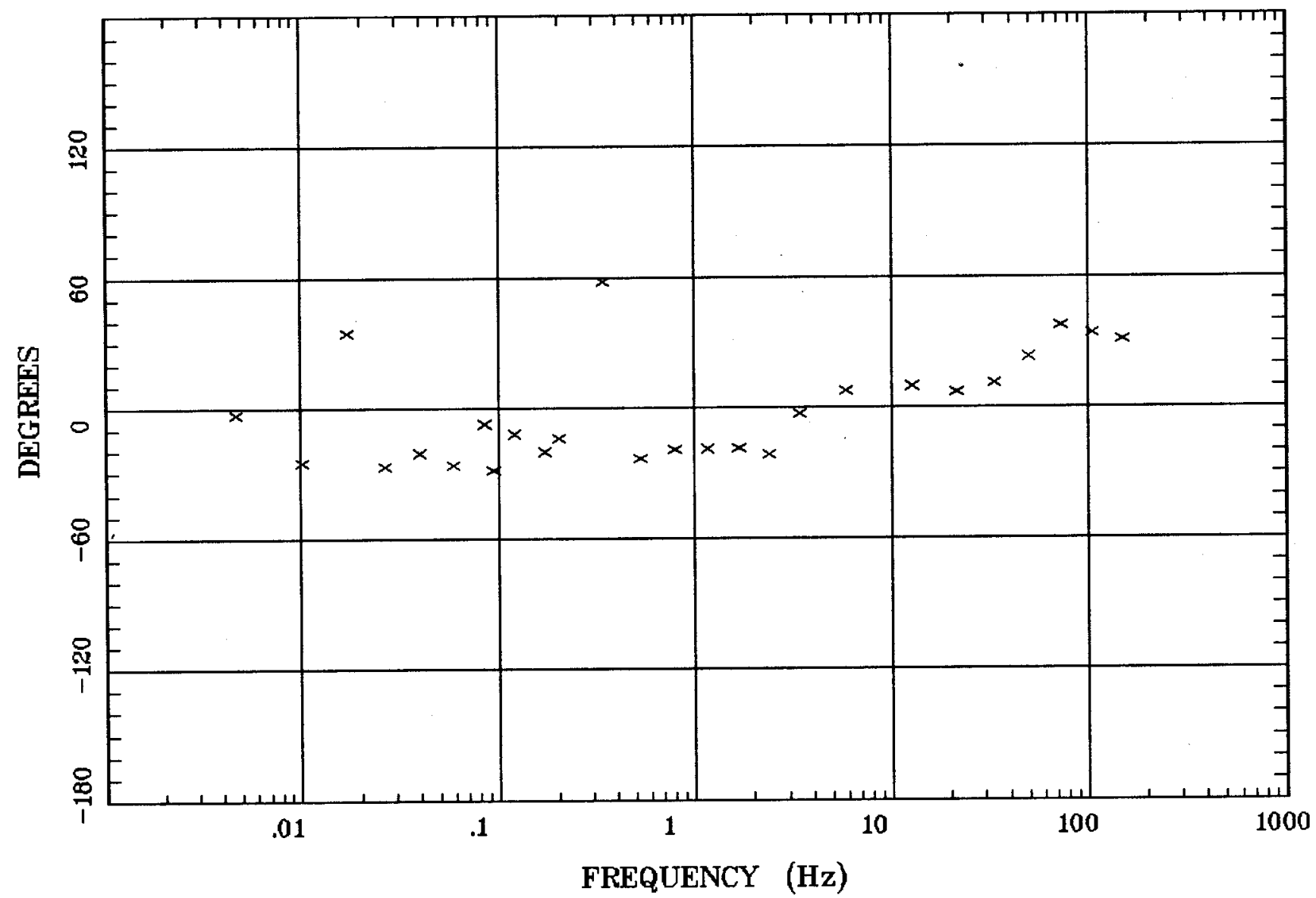

Client:

Remote:

Acquired:

Survey Co:

\section{Rotation:}

Filename: CT17

Channels: Ch1 Ch2 Ch3 Ch4 Ch5 Ch6 Ch7

Plotted: 10:15 Jun 25, 1999

- EMI - ElectroMagnetic Instruments 


\section{Station 17}

\section{IMPEDANCE SKEW}

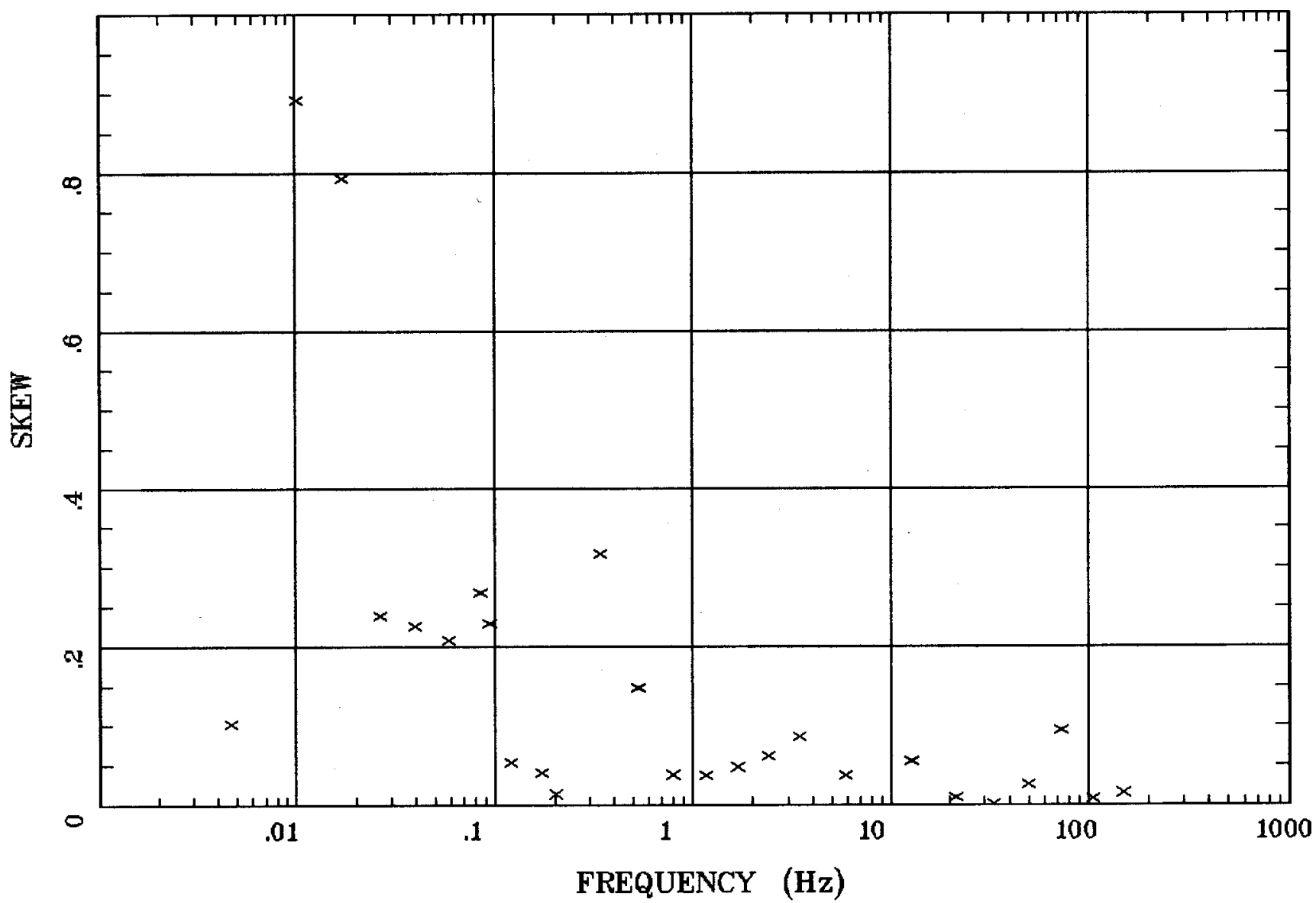

Client:

Remote:

Acquired:

Survey Co:
Rotation:

Filename: CT17

Channels: Ch1 Ch2 Ch3 ch4 Ch5 Ch6 Ch7 Plotted: 10:15 Jun 25, 1999

< EMI - ElectroMagnetic Instruments > 


\section{Station 17}

\section{E MULT Coh.}

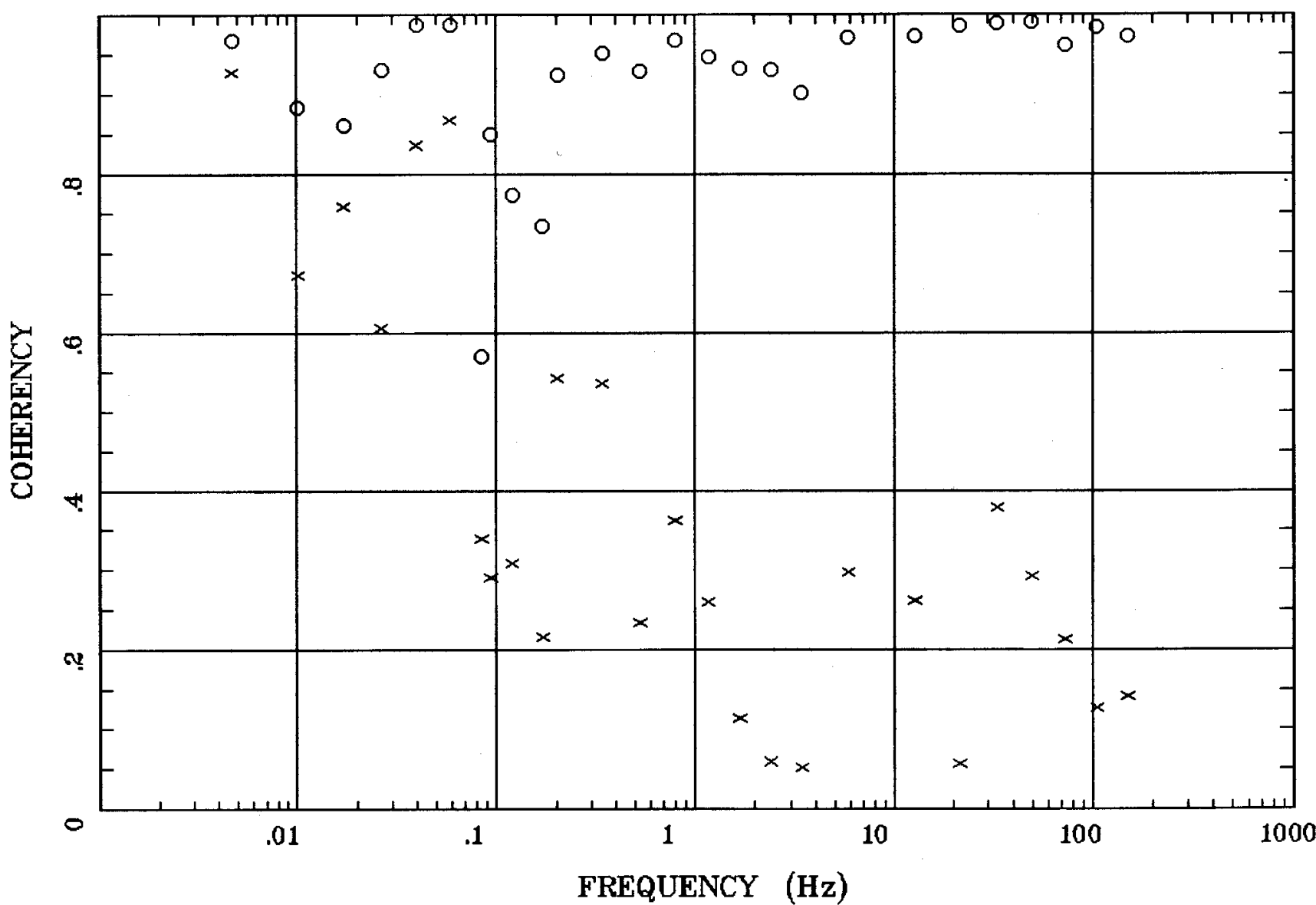

Client:

Rotation:

Remote:

Filename: CT17

Channels: Ch1 Ch2 Ch3 Ch4 Ch5 Ch6 Ch7

Acquired:

Plotted: 10:15 Jun 25, 1999

Survey Co:

< EMI - ElectroMagnetic Instruments > 
Station 17

\section{POLAR PLOTS}

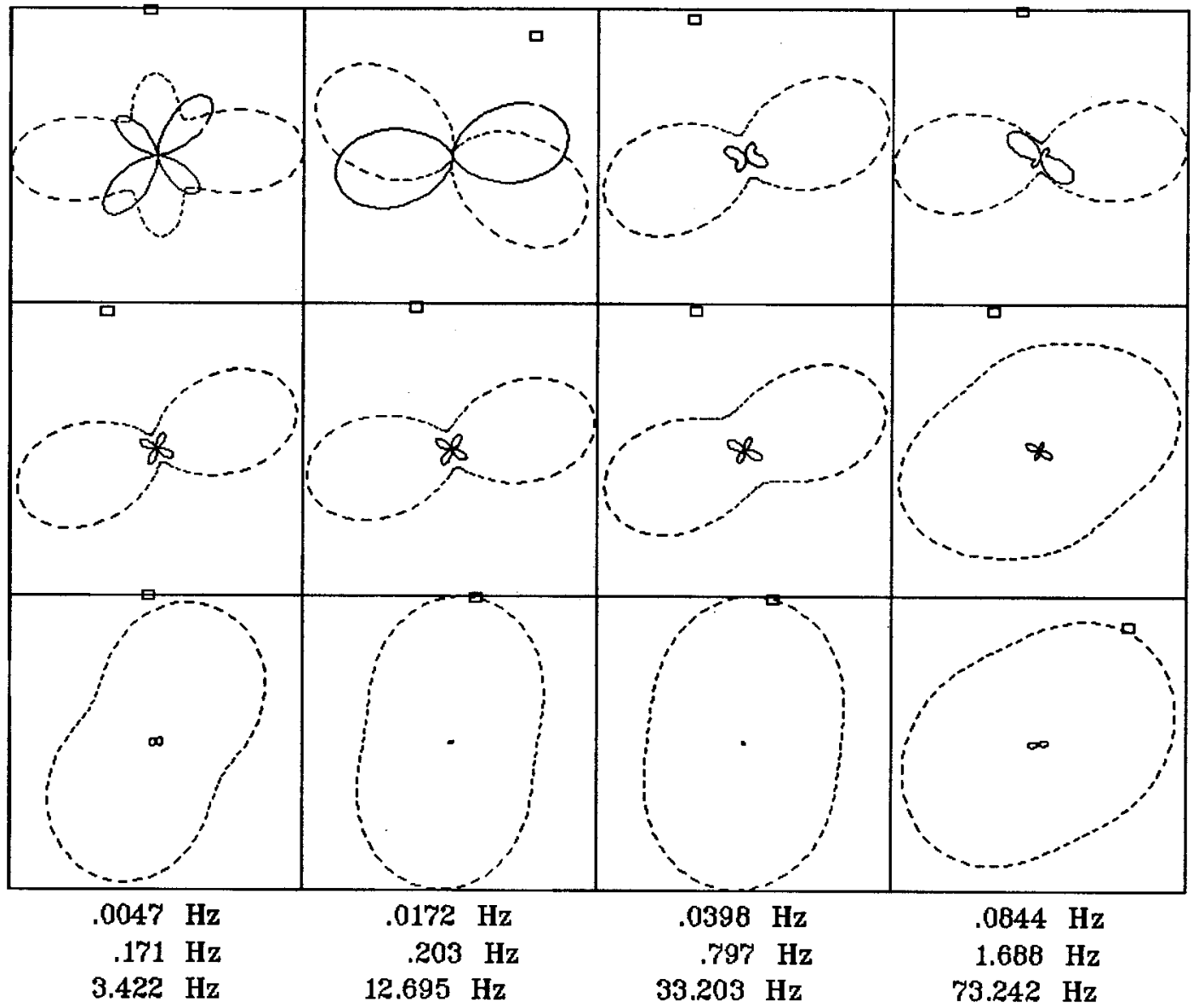

Client:

Remote:

Acquired:

Survey Co:
Rotation:

Filename: CT17

Channels: Ch1 Ch2 Ch3 Ch4 Ch5 Ch6 Ch7 Plotted: 10:15 Jun 25, 1999

< EMI - ElectroMagnetic Instruments > 
Station 11

APPARENT RESISTIVITY Simpson park Mts,NV 100k

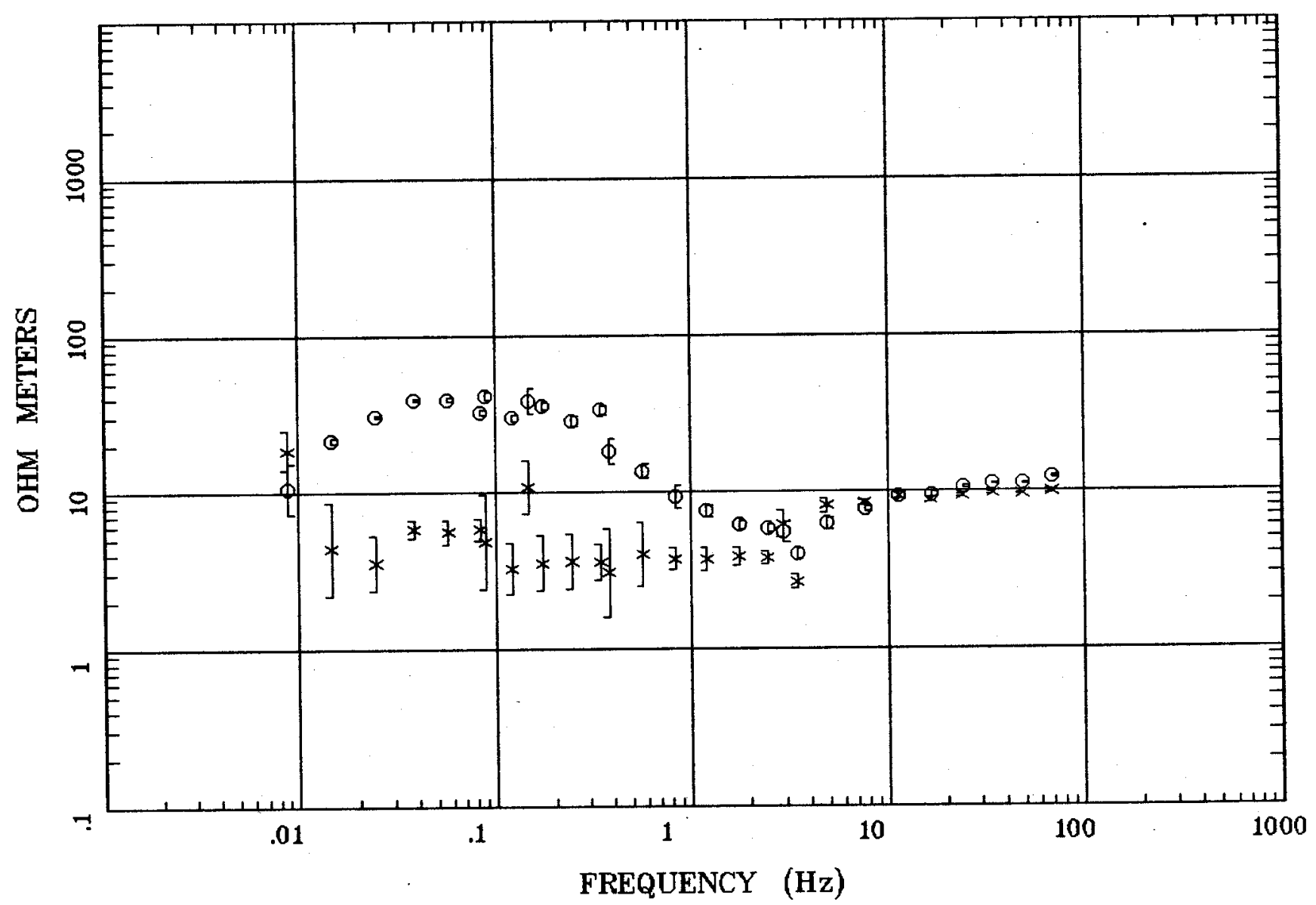

Client:

Remote: none

Acquired: 10:3 Aug 05, 2000

Survey Co:USGS

Rotation:

Filename: nnr11b.avg

Channels: Ch1 Ch2 Ch3 Ch4 Ch5 Ch3 Ch4

Plotted: 09:41 Jan 24, 2001

< EMI - ElectroMagnetic Instruments > 


\section{Station 11}

IMPEDANCE PHASE

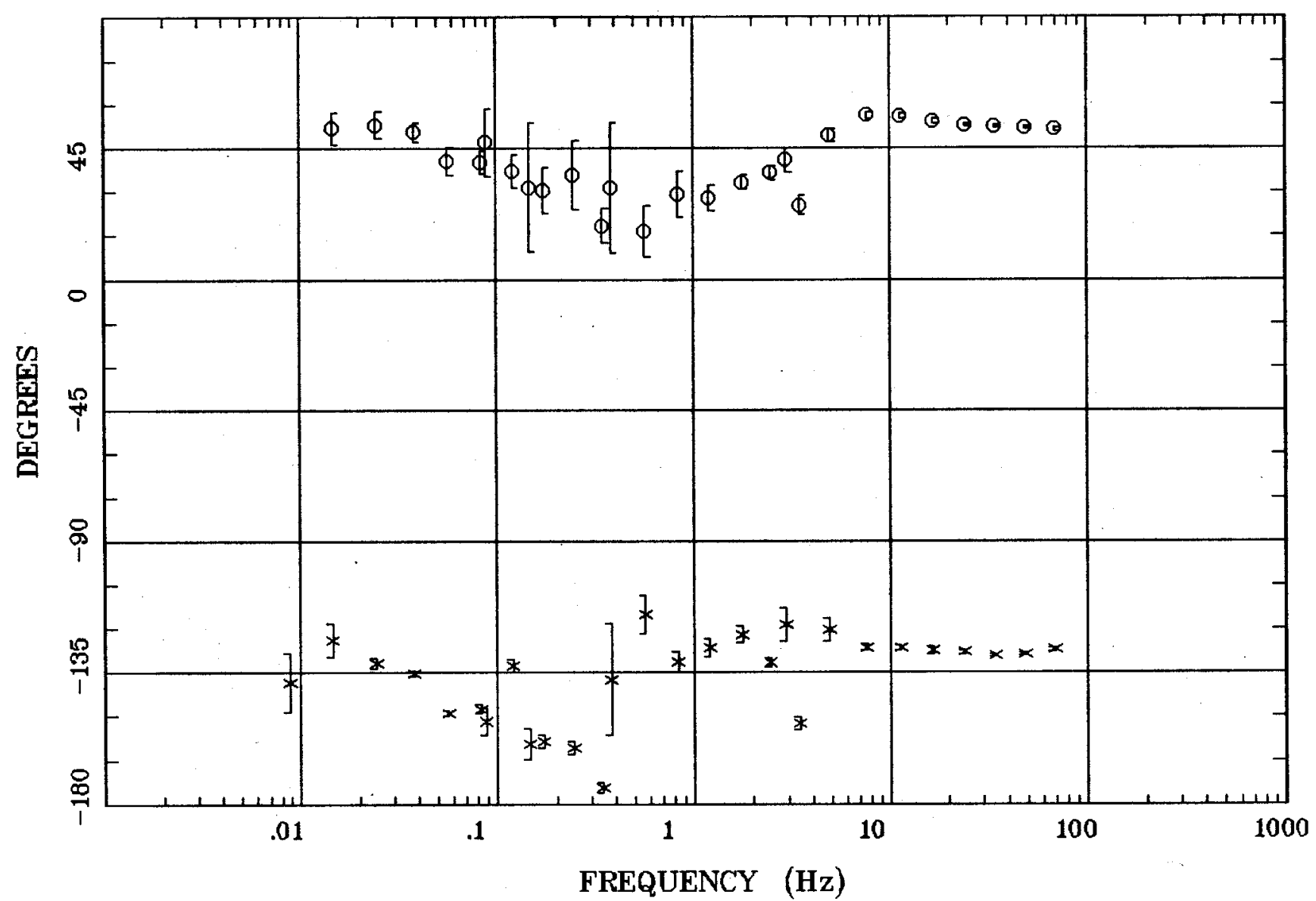

Client:

Remote: none

Acquired: 10:3 Aug 05, 2000

Survey Co:USGS
Simpson park Mts,NV 100k

Rotation:

Filename: nnrlib.avg

Channels: Ch1 Ch2 Ch3 Ch4 Ch5 Ch3 Ch4

Plotted: 09:41 Jan 24, 2001

< EMI - ElectroMagnetic Instruments > 
Station 11

\section{ROTATION ANGLE}

Simpson park Mts,NV 100k

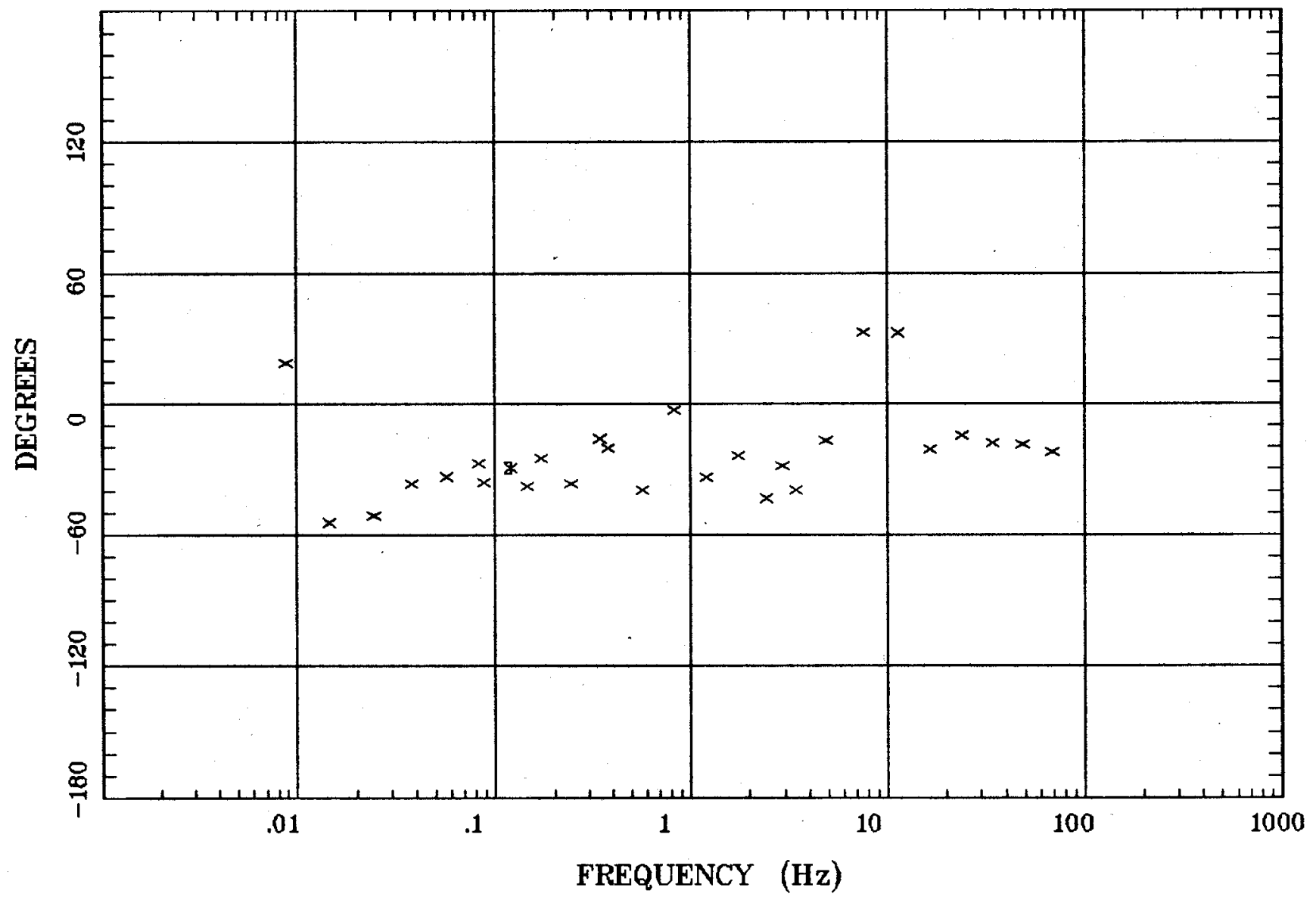

Client:

Rotation:

Remote: none

Filename: nnr11b.avg

Acquired: 10:3 Aug 05, 2000

Channels: Ch1 Ch2 Ch3 Ch4 Ch5 Ch3 Ch4

Platted: 09:42 Jan 24, 2001

Survey Co:USGS

< EMI - ElectroMagnetic Instruments > 


\section{Station 11}

IMPEDANCE SKEW

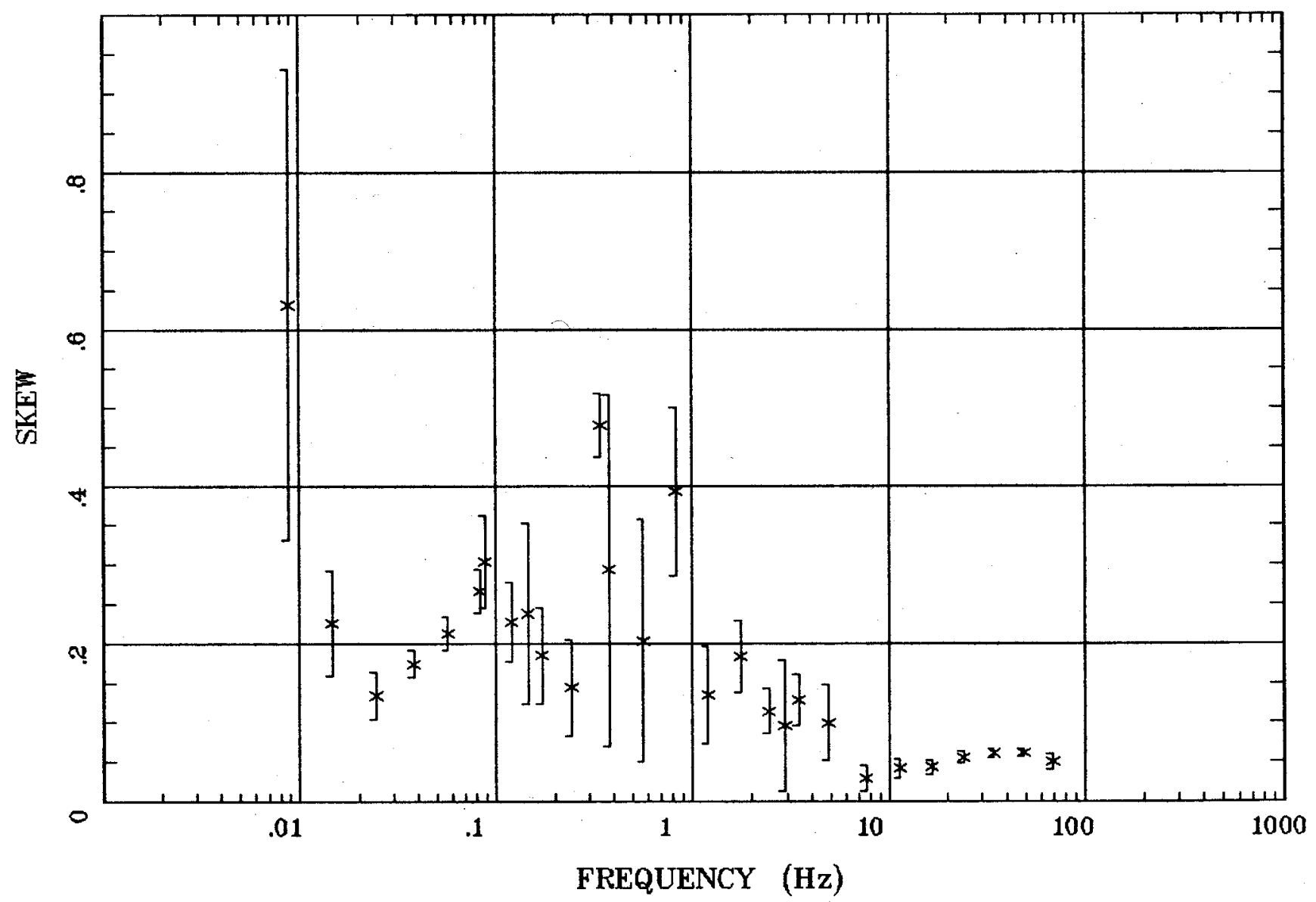

Client:

Remote: none

Acquired: 10:3 Aug 05, 2000 Survey Co:USGS

\section{Rotation:}

Filename: nnrilb.avg

Channels: Ch1 Ch2 Ch3 Ch4 Ch5 Ch3 Ch4 Plotted: 09:42 Jan 24, 2001

< EMI - ElectroMagnetic Instruments > 


\section{Station 11}

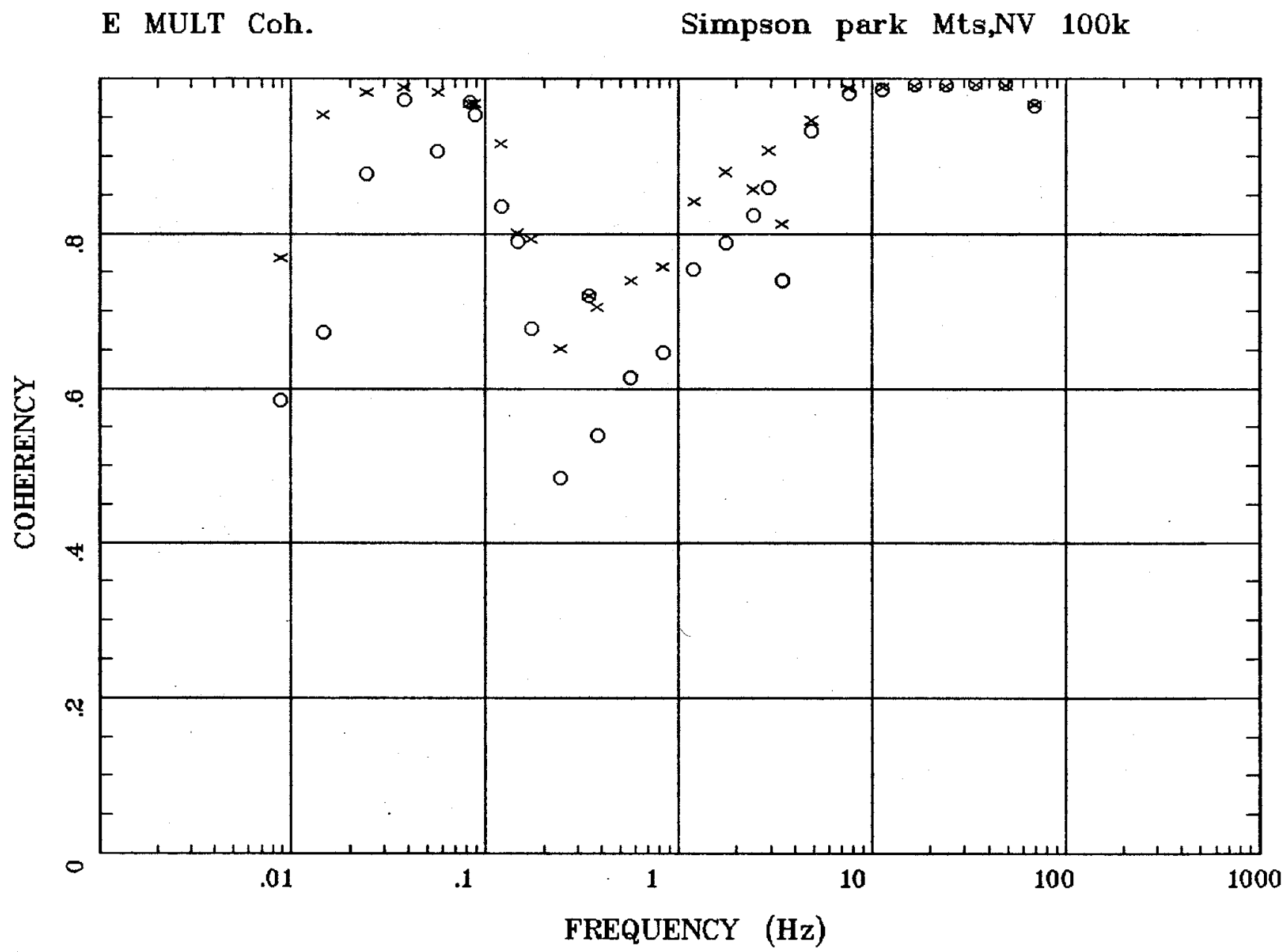

Client:

Remote: none

Acquired: 10:3 Aug 05, 2000

Survey Co:USGS
Rotation:

Filename: nnr11b.avg

Channels: Ch1 Ch2 Ch3 Ch4 Ch5 Ch3 Ch4

Plotted: 09:42 Jan 24, 2001

< EMI - ElectroMagnetic Instruments > 


\section{Station 11}

POLAR PLOTS

Simpson park Mts,NV 100k

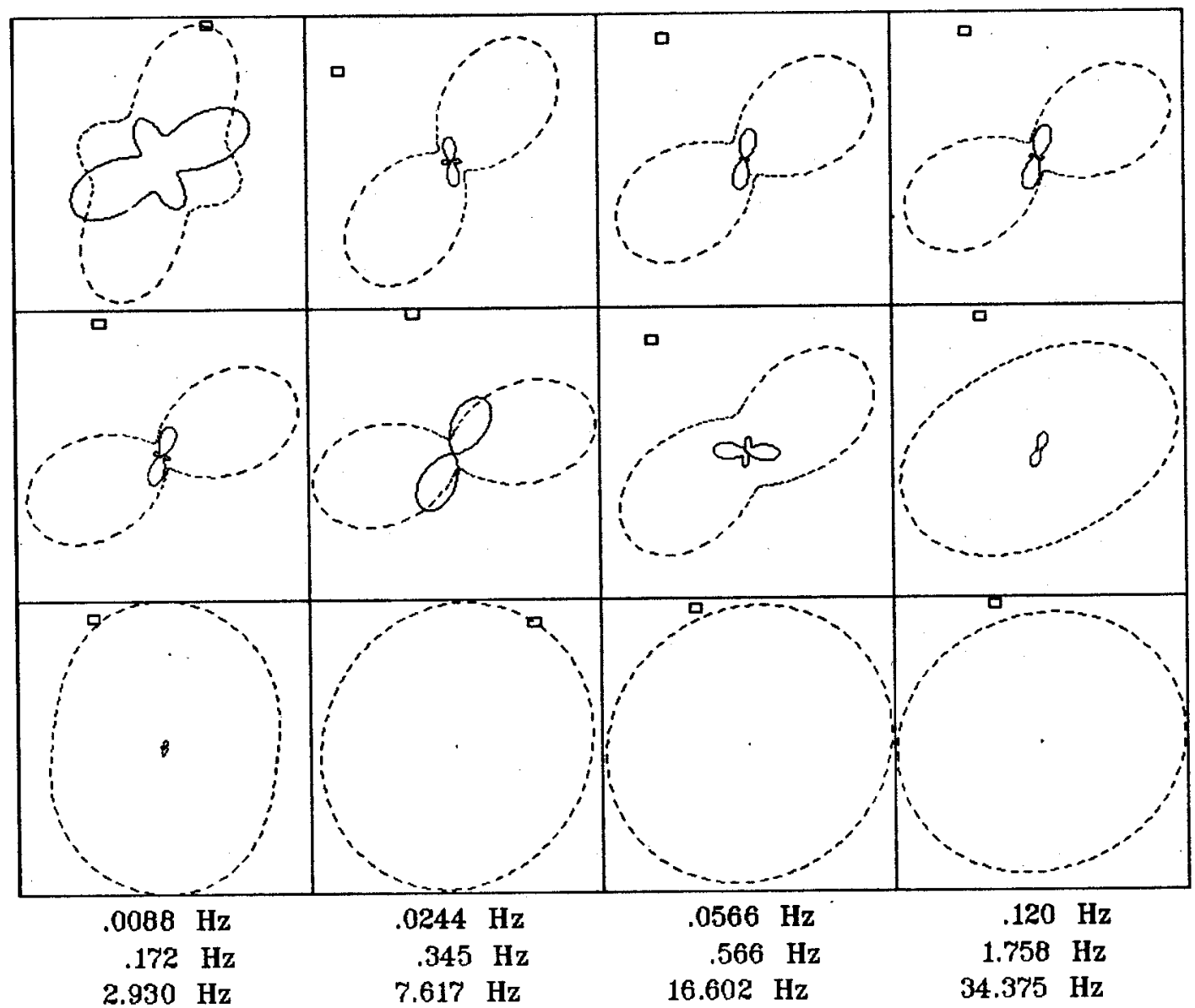

\section{Client:}

Remote: none

Acquired: 10:3 Aug 05, 2000

Survey Co:USGS
Rotation:

Filename: nnr11b.avg

Channels: Ch1 Ch2 Ch3 Ch4 Ch5 Ch3 Ch4

Plotted: 09:42 Jan 24, 2001

< EMI - ElectroMagnetic Instruments > 


\section{Station 11}

\section{TIPPER MAGNITUDE}

Simpson park Mts,NV 100k

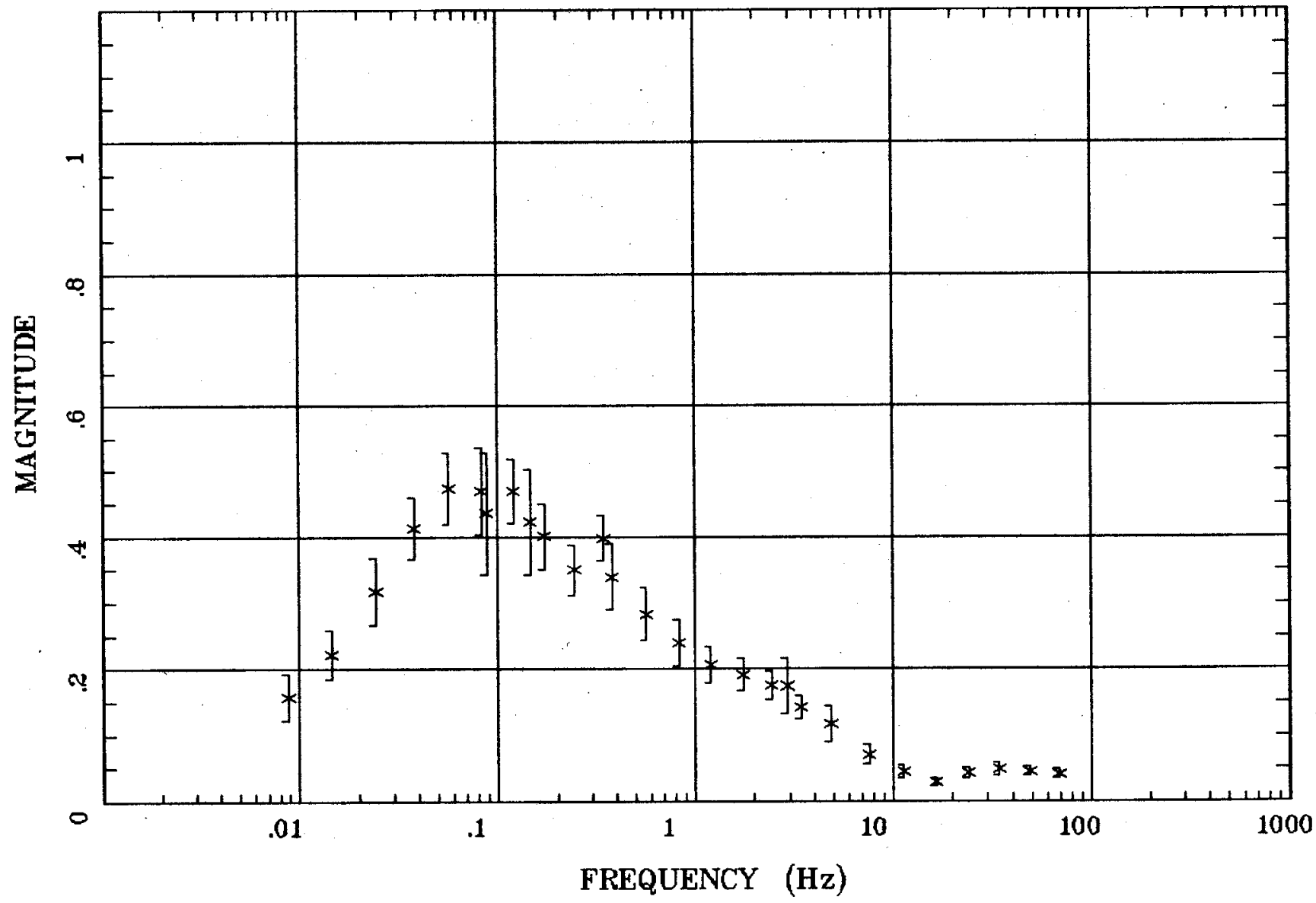

Rotation:

Client:

Remote: none

Acquired: 10:3 Aug 05, 2000

Survey Co:USGS
Filename: nnr11b.avg

Channels: Ch1 Ch2 Ch3 Ch4 Ch5 Ch3 Ch4

Plotted: 09:42 Jan 24, 2001

$<$ EMI - ElectroMagnetic Instruments 


\section{Station 11}

TIPPER STRIKE

Simpson park Mts,NV 100k

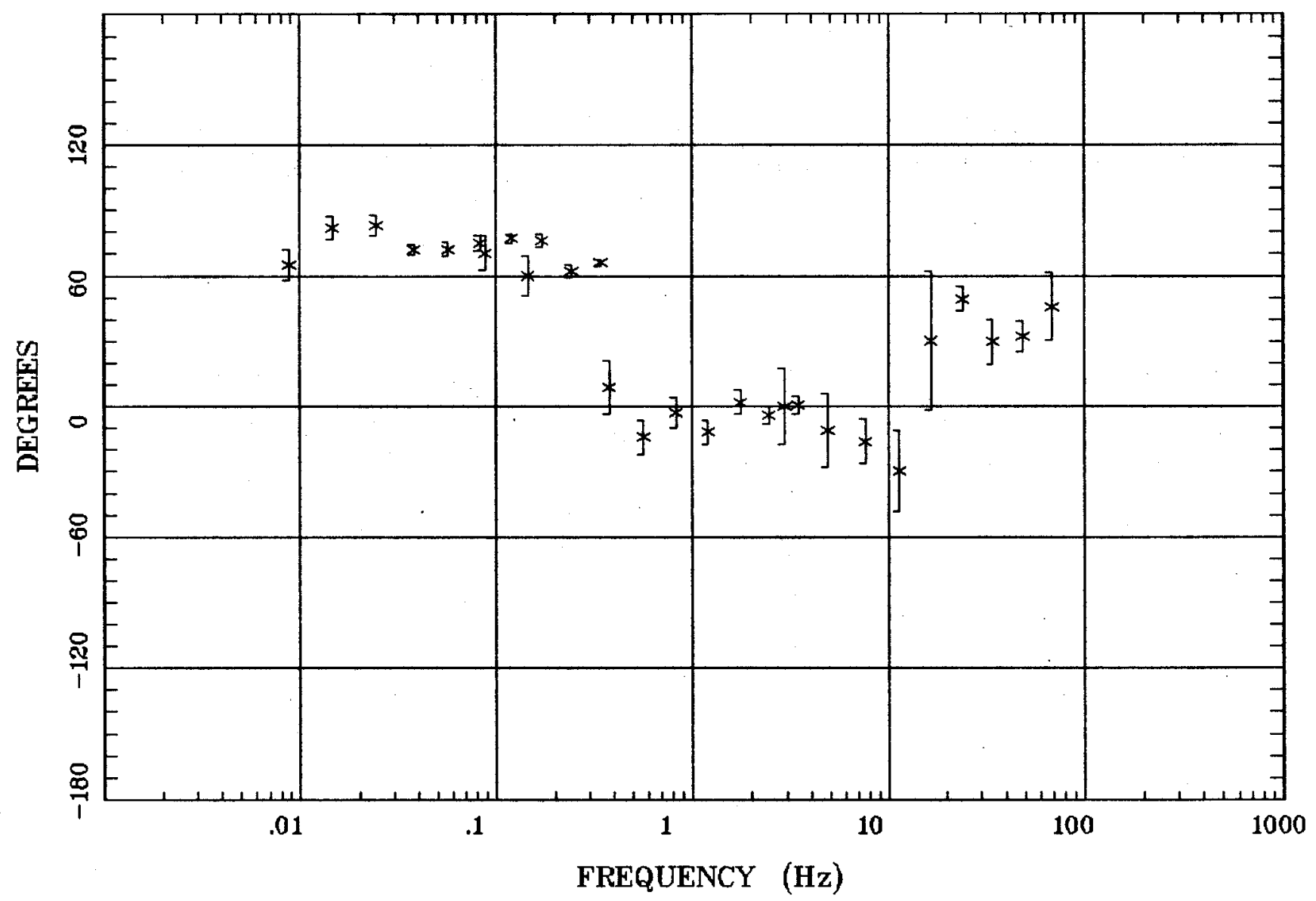

Client:

Remote: none

Acquired: 10:3 Aug 05, 2000

Survey Co:USGS

Rotation:

Filename: nnr11b.avg

Channels: Ch1 Ch2 Ch3 Ch4 Ch5 Ch3 Ch4

Plotted: 09:42 Jan 24, 2001

< EMI - ElectroMagnetic Instruments > 


\section{Station 11}

\section{HzHx.x Coh HzHy.o Simpson park Mts,NV 100k}

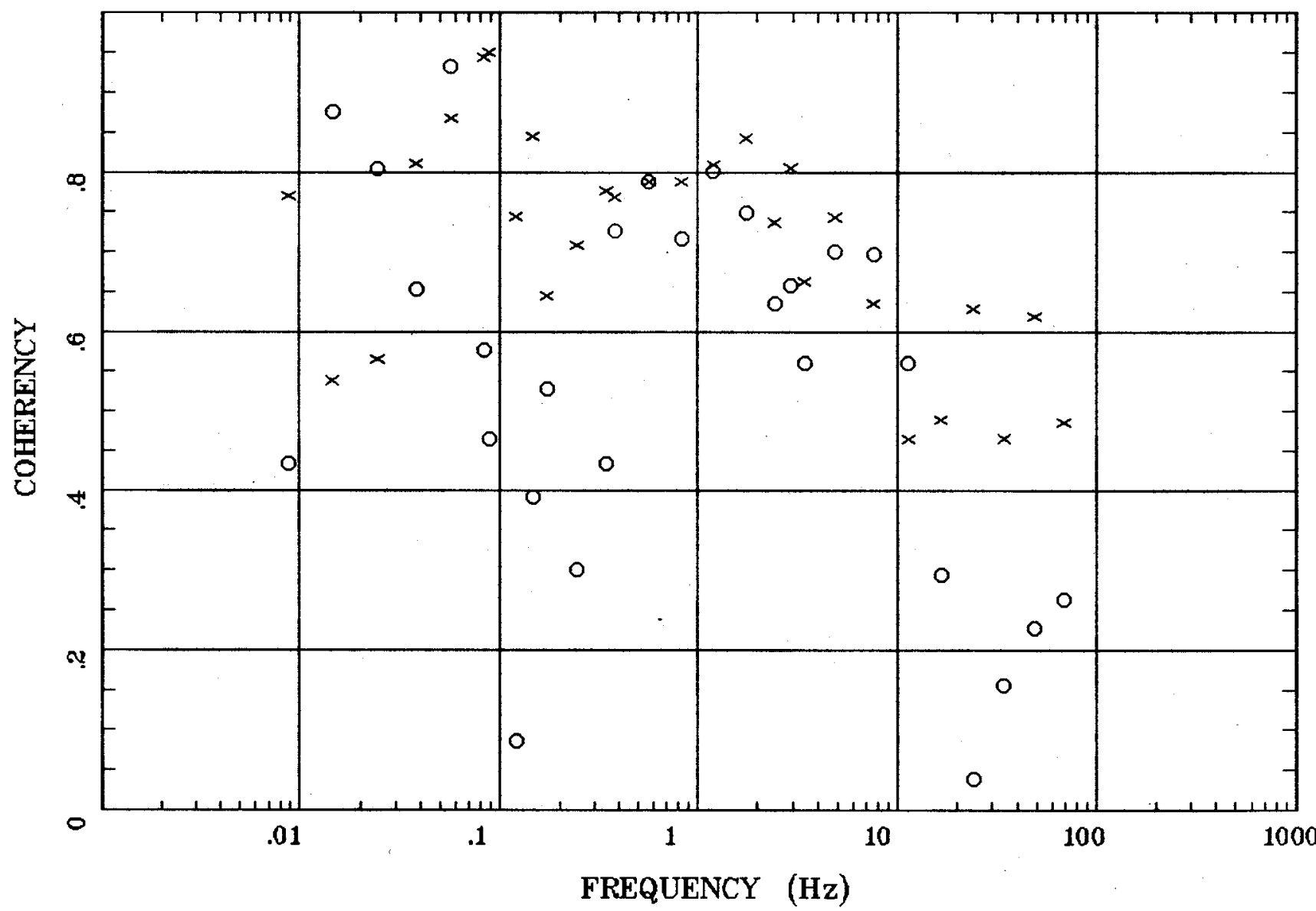

Client:

Rotation:

Remote: none

Acquired: 10:3 Aug 05, 2000

Filename: nnr11b.avg

Channels: Ch1 Ch2 Ch3 Ch4 Ch5 Ch3 Ch4

Plotted: 09:42 Jan 24, 2001

Survey Co:USGS

< EMI - ElectroMagnetic Instruments > 
APPARENT RESISTIVITY

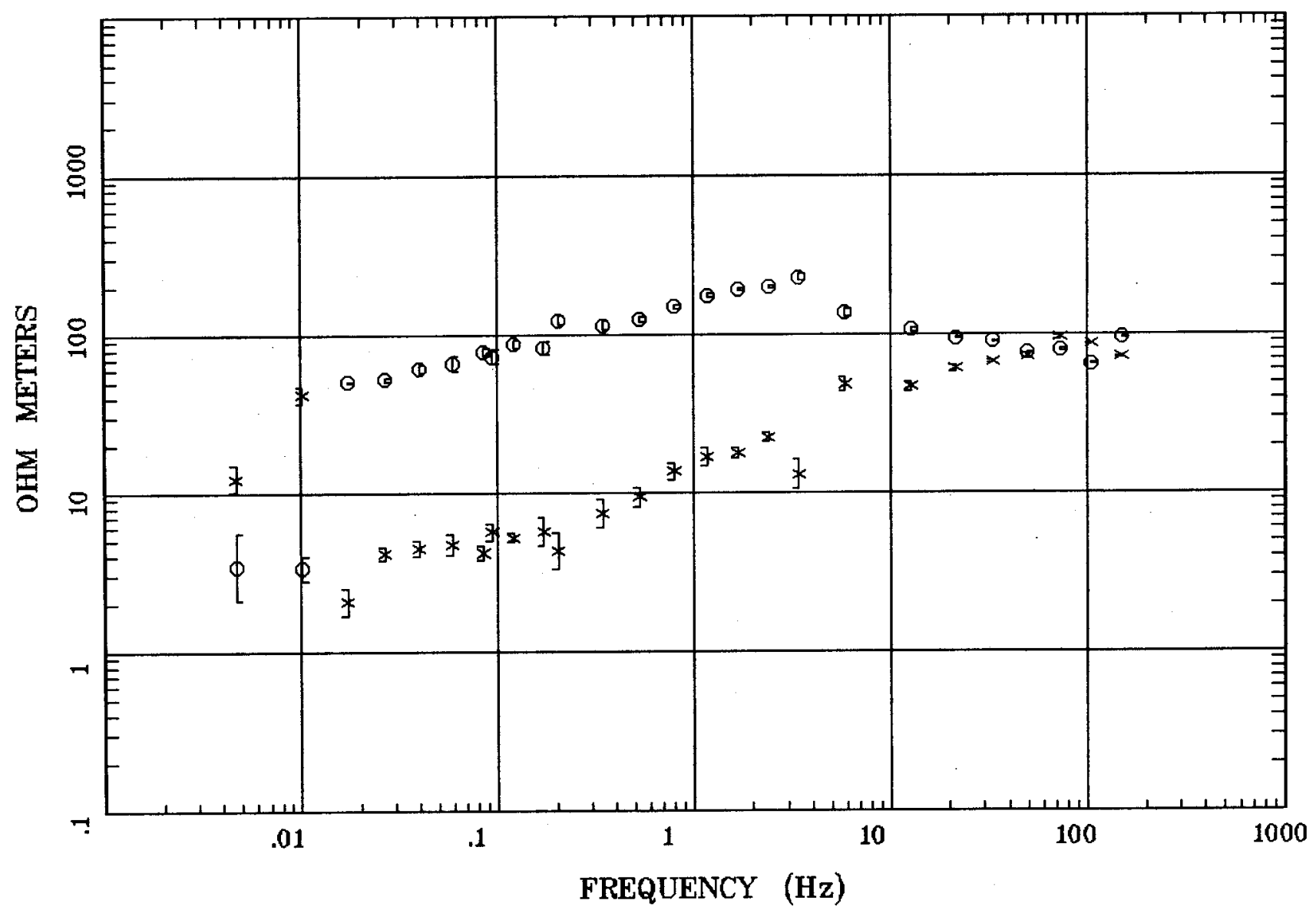

Client:

Remote:

Acquired:

Survey Co:
Rotation:

Filename: NNR10A

Channels: Ch1 Ch2 Ch3 Ch4 Ch5 Ch6 Ch7 Plotted: 17:49 Jun 24, 1999

< EMI - ElectroMagnetic Instruments > 


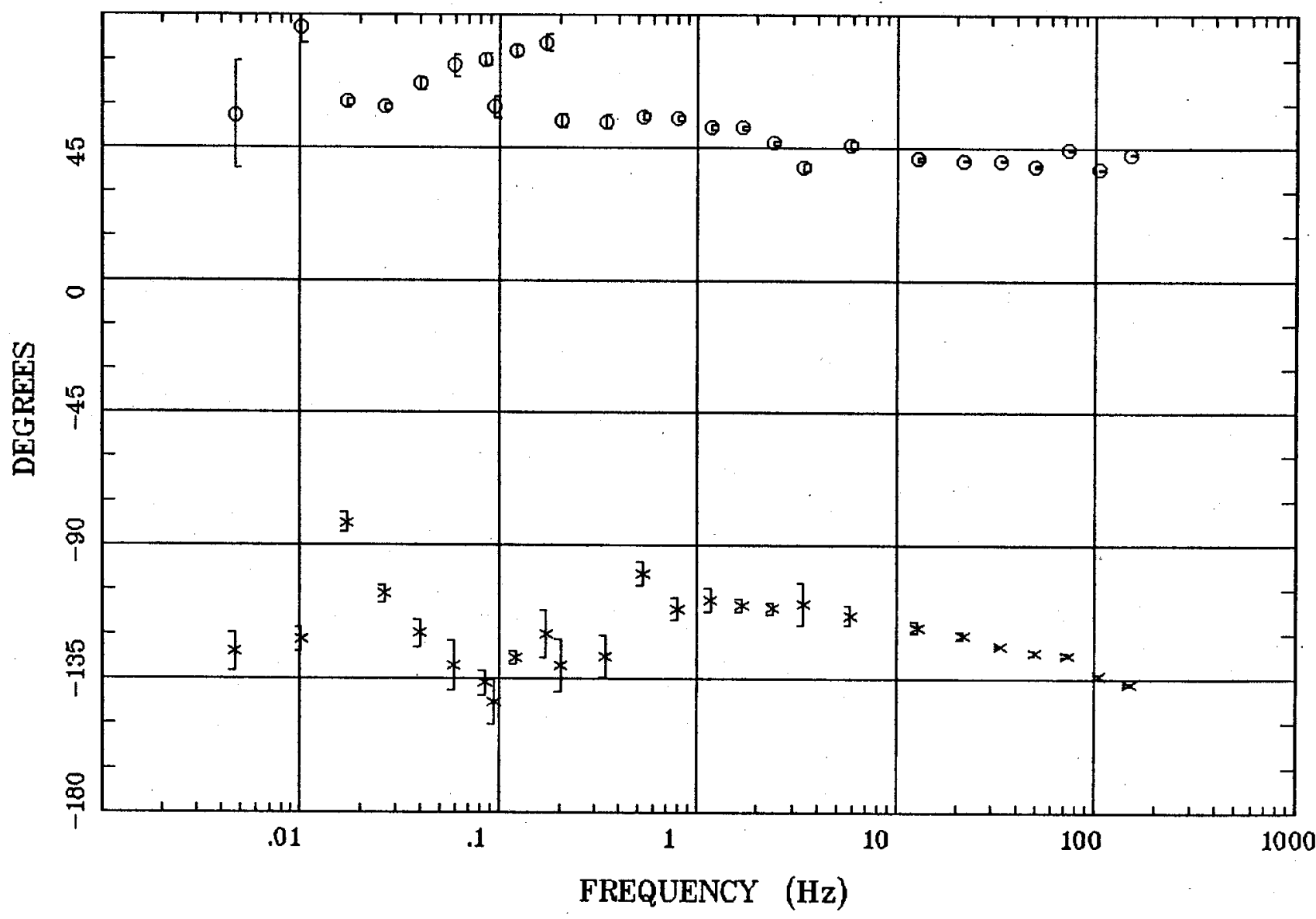

Client:

Remote:

Acquired:

Survey Co:
Rotation:

Filename: NNR10A

Channels: Ch1 Ch2 Ch3 Ch4 Ch5 Ch6 Ch7 Plotted: 17:49 Jun 24, 1999

< EMI - ElectroMagnetic Instruments 
Station 10

\section{ROTATION ANGLE}

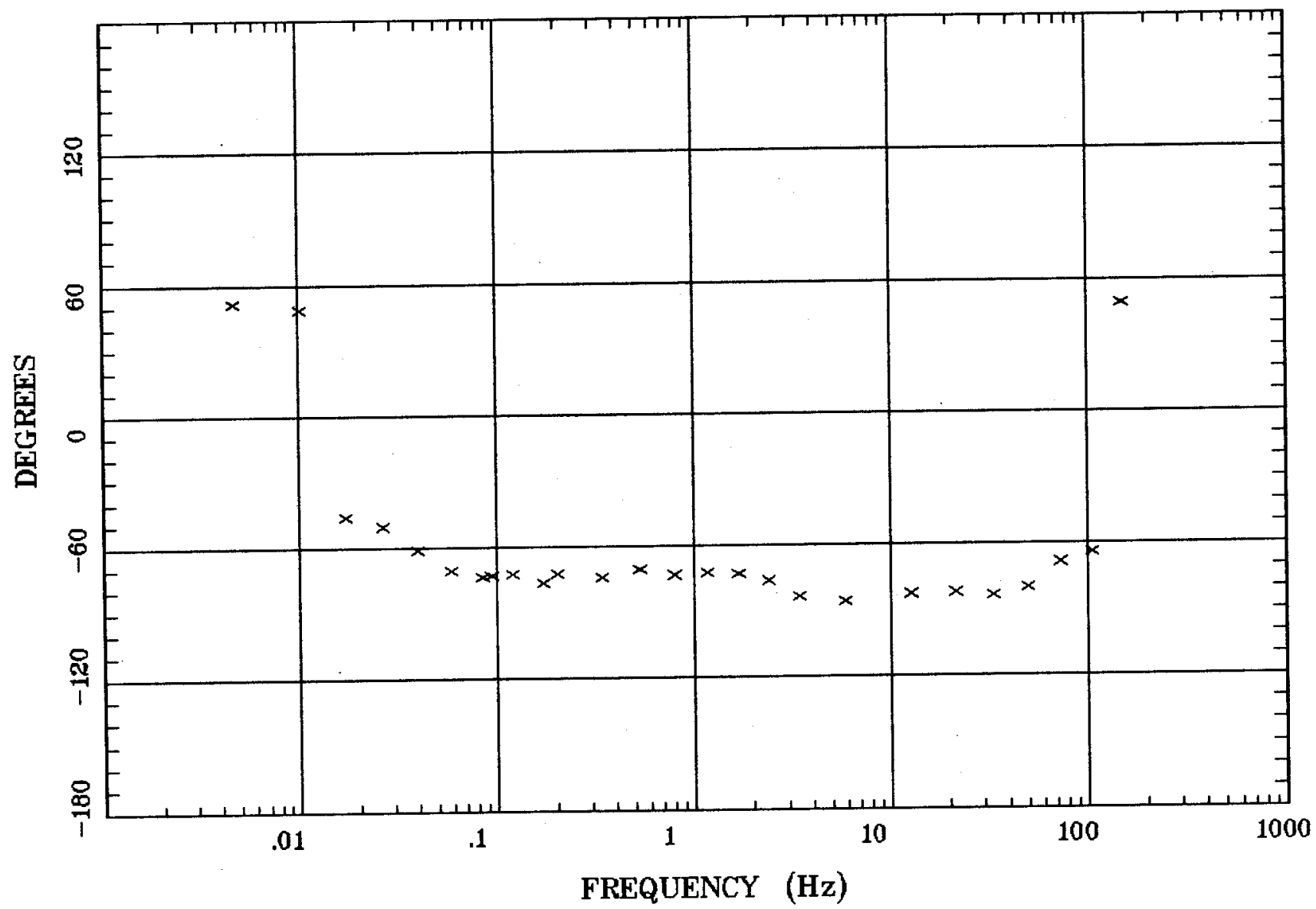

Client:

Remote:

Acquired:

Survey Co:
Rotation:

Filename: NNR10A

Channels: Ch1 Ch2 Ch3 Ch4 Ch5 Ch6 Ch7

Plotted: 17:49 Jun 24, 1999

< EMI - ElectroMagnetic Instruments 
IMPEDANCE SKEW

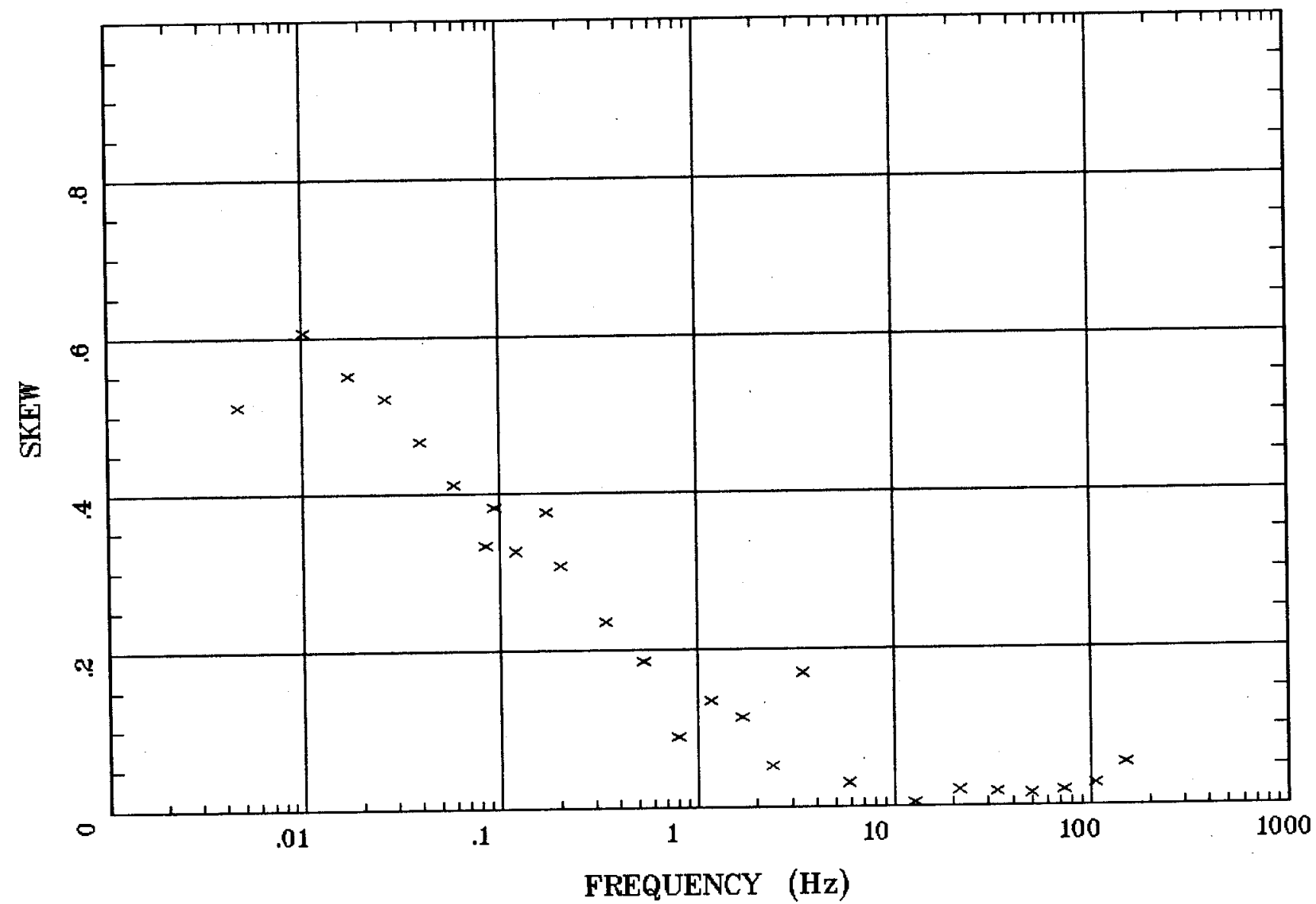

Rotation:

Client:

Filename: NNR10A

Remote:

Channels: Ch1 Ch2 Ch3 Ch4 Ch5 Ch6 Ch7

Plotted: 17:49 Jun 24, 1999

Acquired:

$<$ EMI - ElectroMagnetic Instruments 


\section{Station 10}

E MULT Coh.

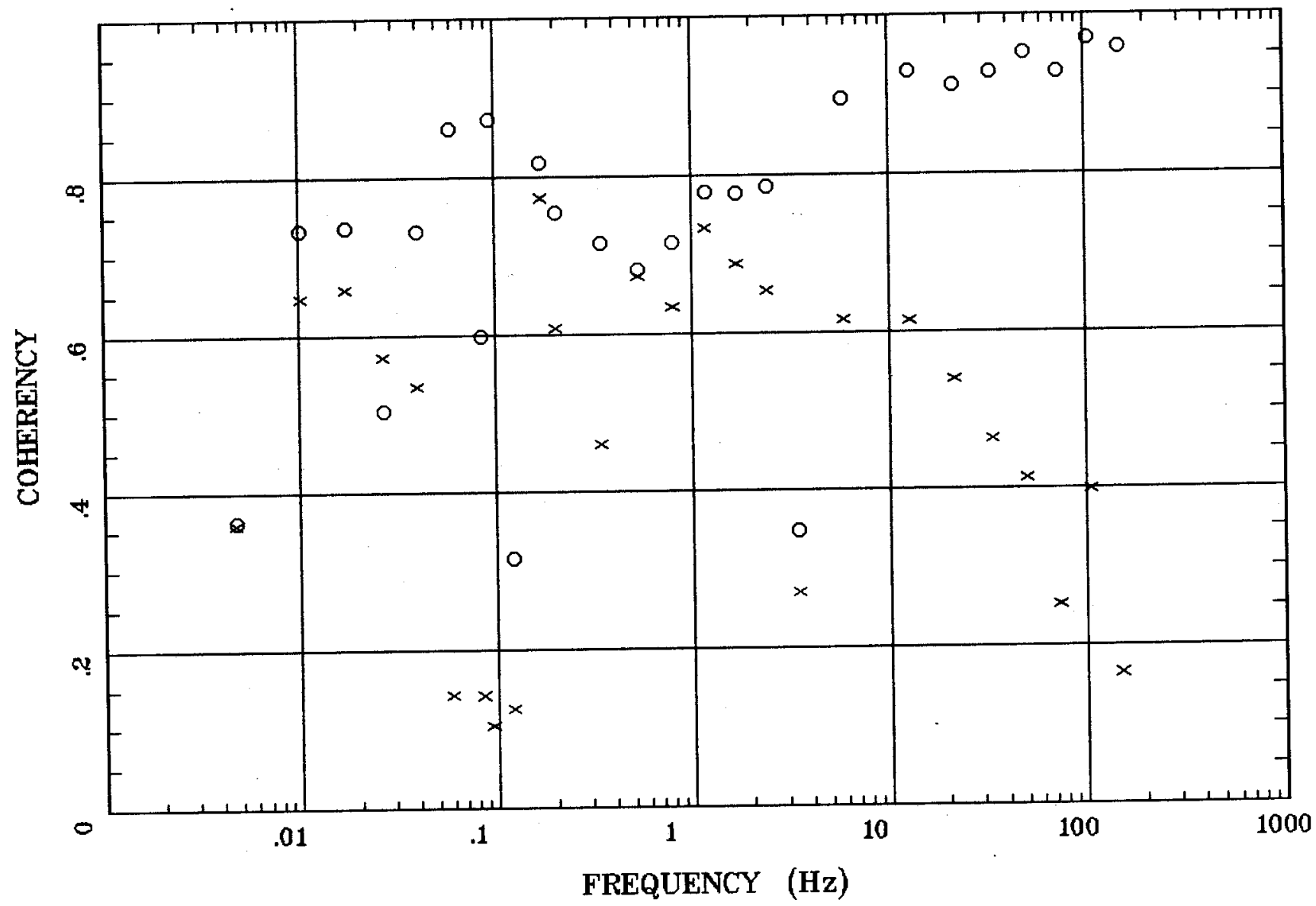

Rotation:

Client:

Filename: NNR10A

Remote:

Channels: Ch1 Ch2 Ch3 Ch4 Ch5 Ch6 Ch7

Platted: $17: 49$ Jun 24, 1999

Acquired:

< EMI - ElectroMagnetic Instruments > 


\section{Station 10}

POLAR PLOTS

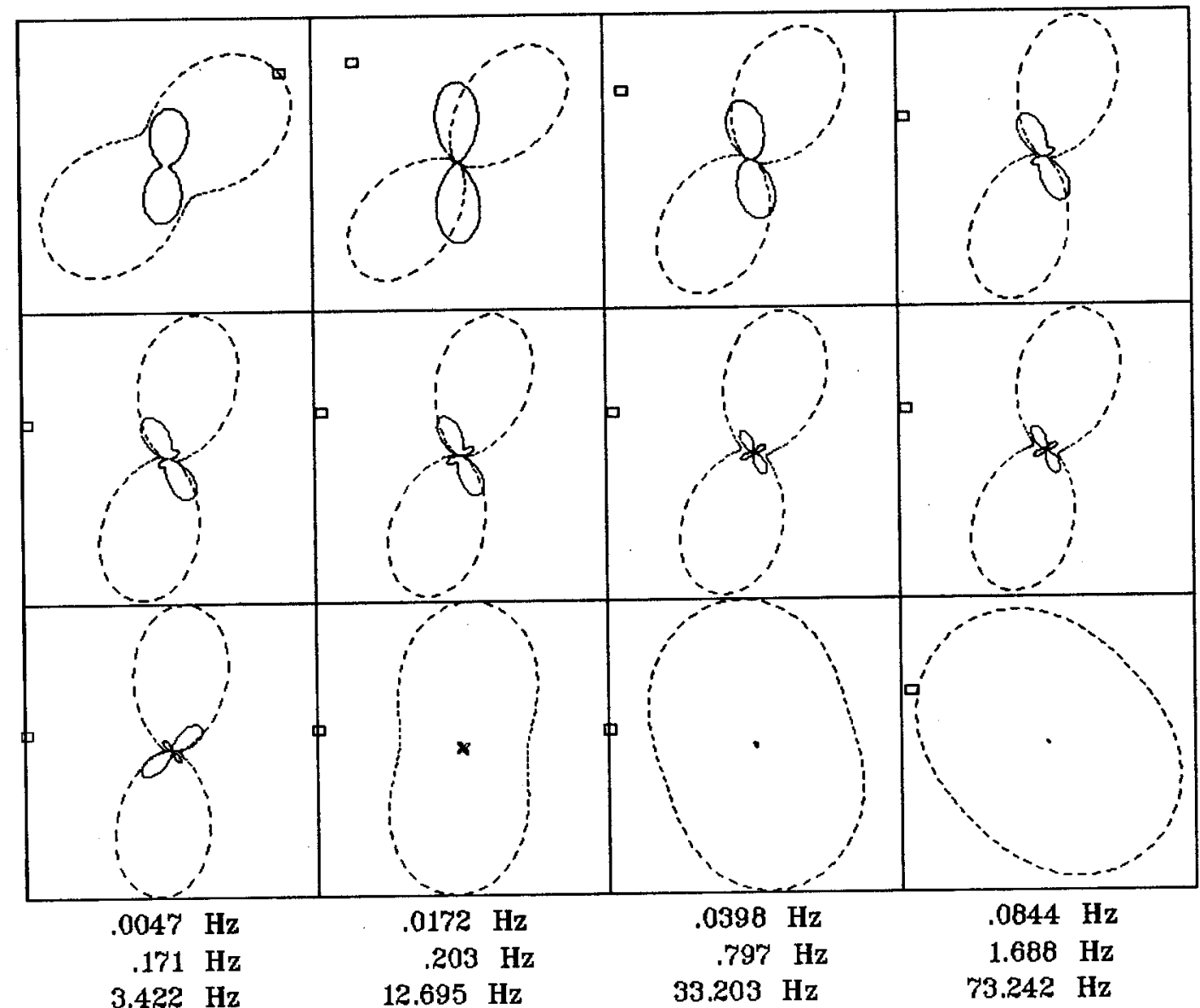

Client:

Remote:

Acquired:

Survey Co:

Rotation:

Filename: NNR10A

Channels: Ch1 Ch2 Ch3 Ch4 Ch5 Ch6 Ch7

Platted: $17: 49$ Jun 24, 1999

< EMI - ElectroMagnetic Instruments 


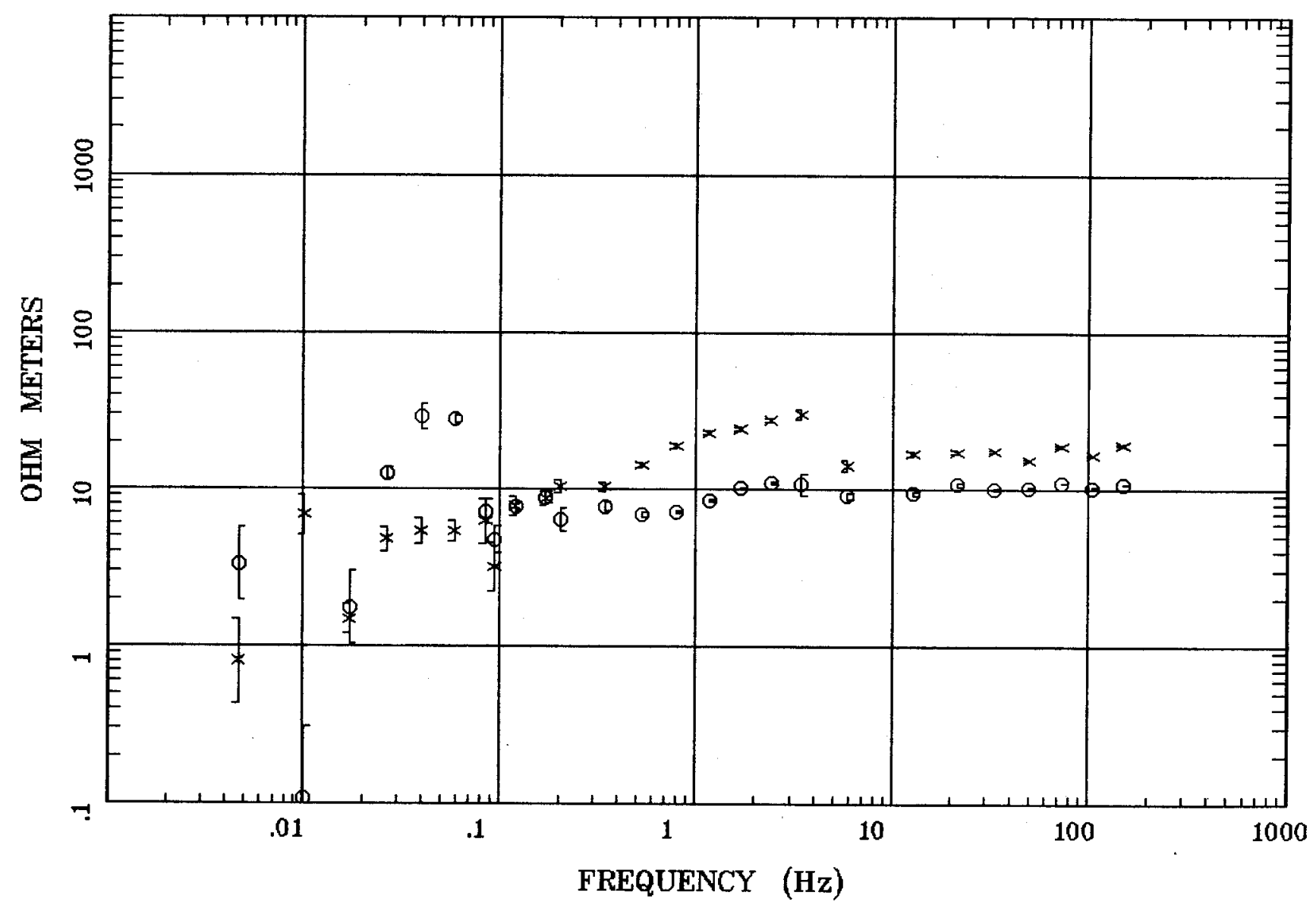

Client:

Remote:

Acquired:

Survey Co:
Rotation:

Filename: NNR06

Channels: Ch1 Ch2 Ch3 Ch4 Ch5 Ch6 Chr

Plotted: 18:15 Jun 24, 1999

< EMI - ElectroMagnetic Instruments > 
Station 06

\section{IMPEDANCE PHASE}

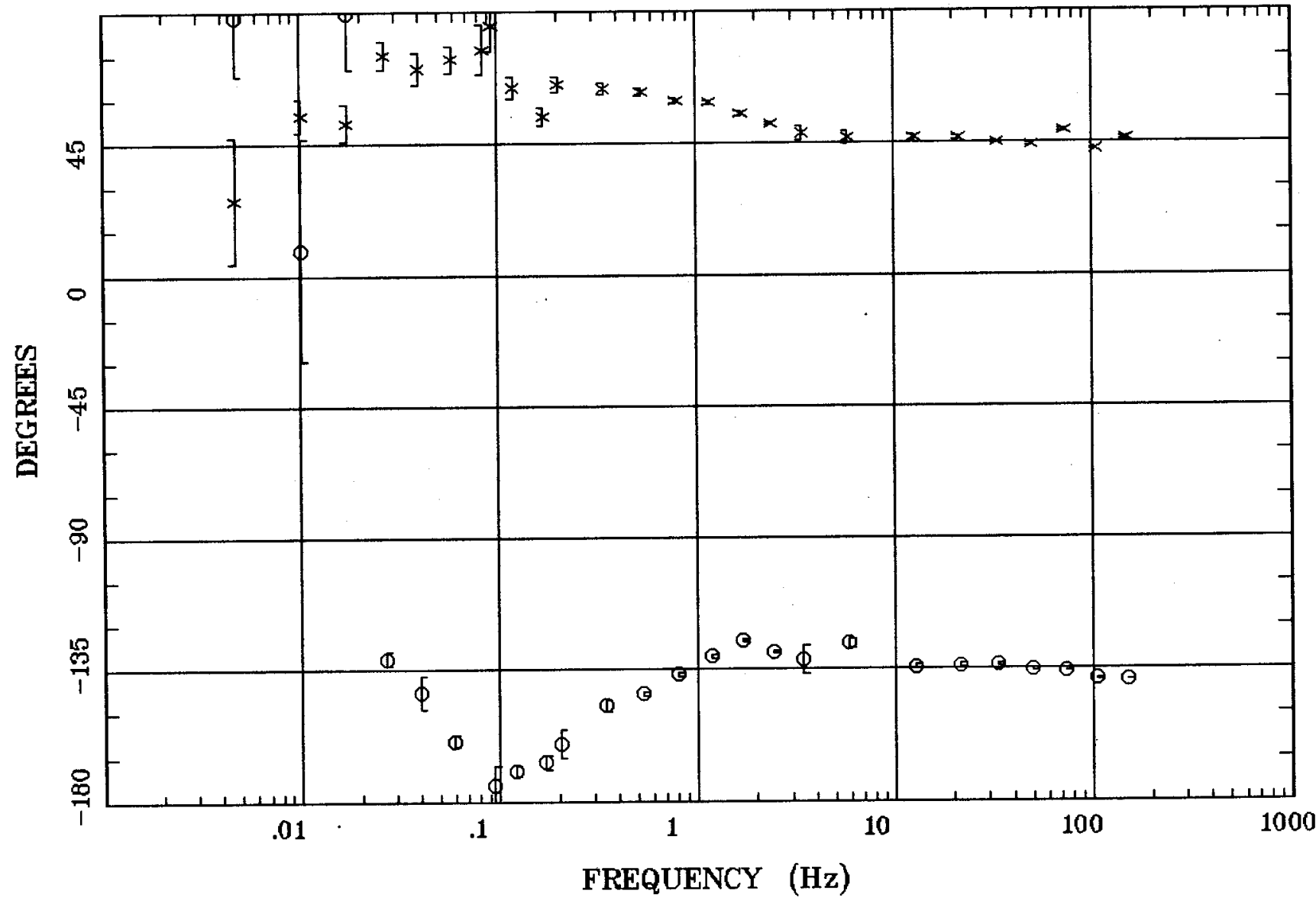

Client:

Remote:

Acquired:

Survey $\mathrm{Co}$
Rotation:

Filename: NNR06

Channels: Ch1 Ch2 Ch3 Ch4 Ch5 Ch6 Ch7

Plotted: 18:15 Jun 24, 1999

< EMI - ElectroMagnetic Instruments > 


\section{Station 06}

\section{ROTATION ANGLE}

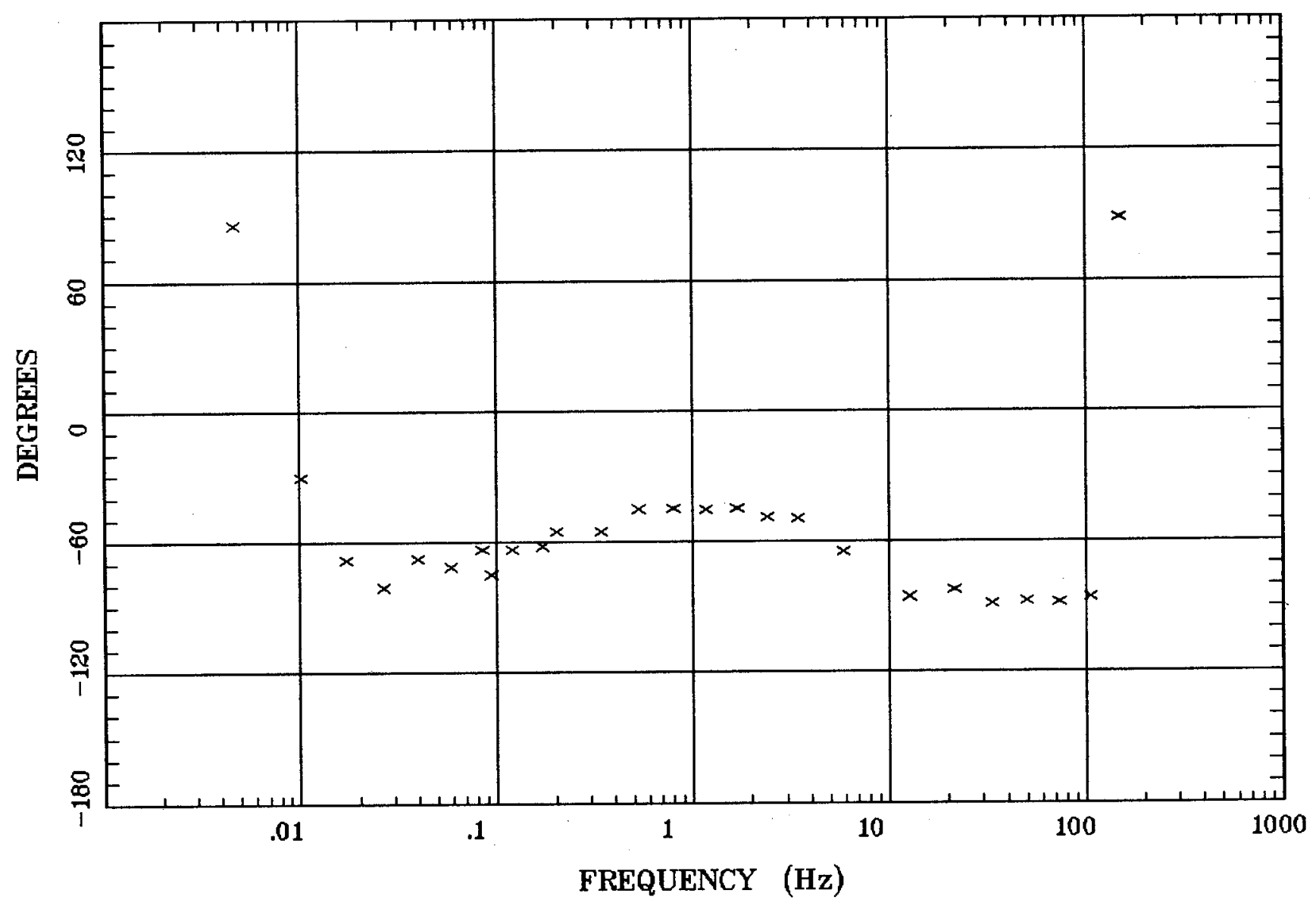

Client:

Remote:

Acquired:

Survey Co:

Rotation:

Filename: NNR06

Channels: Ch1 Ch2 Ch3 Ch4 Ch5 Ch6 Ch7

Plotted: 18:15 Jun 24, 1999

< EMI - ElectroMagnetic Instruments > 


\section{Station 06}

\section{IMPEDANCE SKEW}

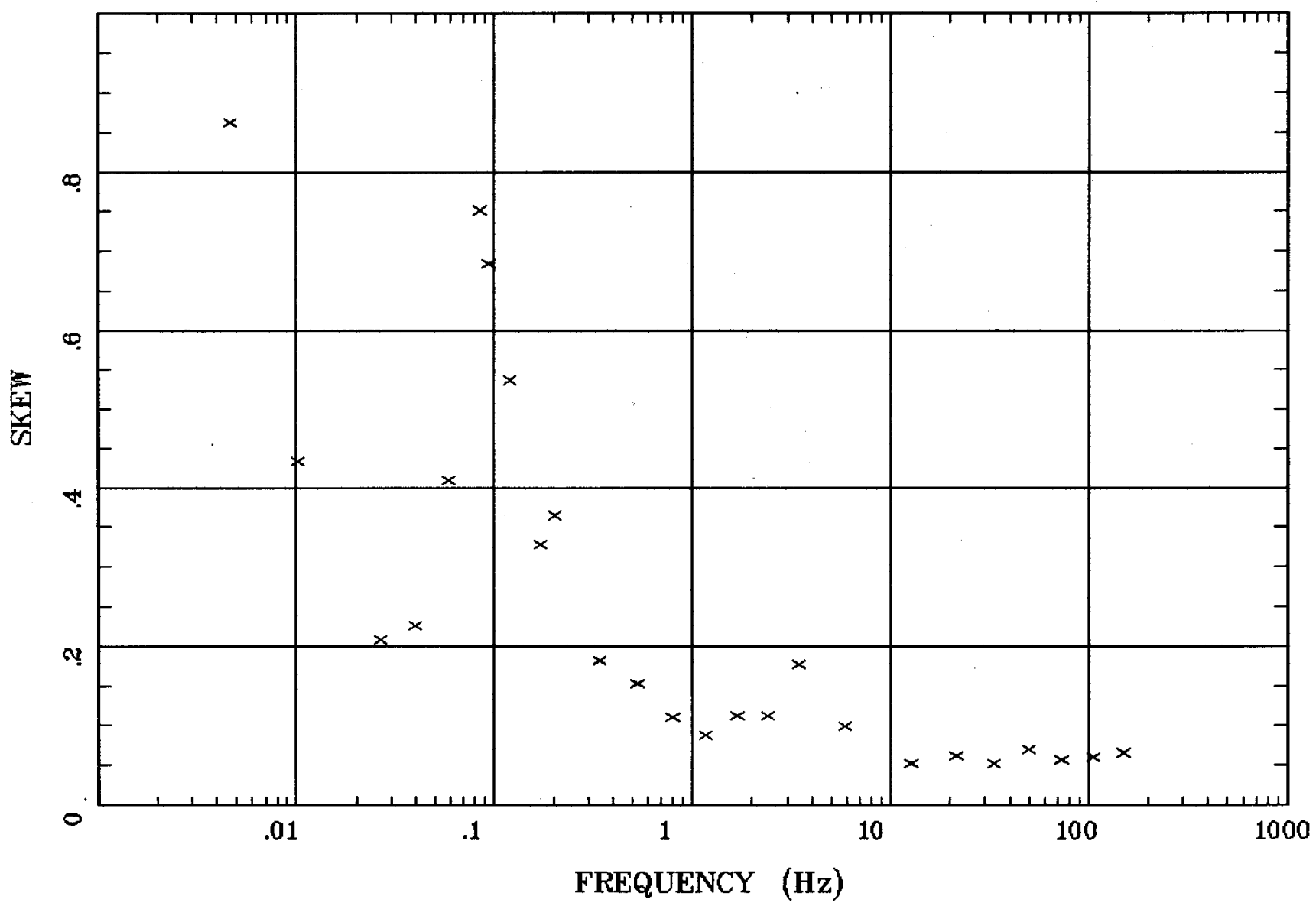

Client:

Remote:

Acquired:

Survey Co:
Rotation:

Filename: NNR06

Channels: Ch1 Ch2 Ch3 Ch4 Ch5 Ch6 Ch7

Plotted: 18:15 Jun 24, 1999

< EMI - ElectroMagnetic Instruments > 


\section{Station 06}

\section{E MULT Coh.}

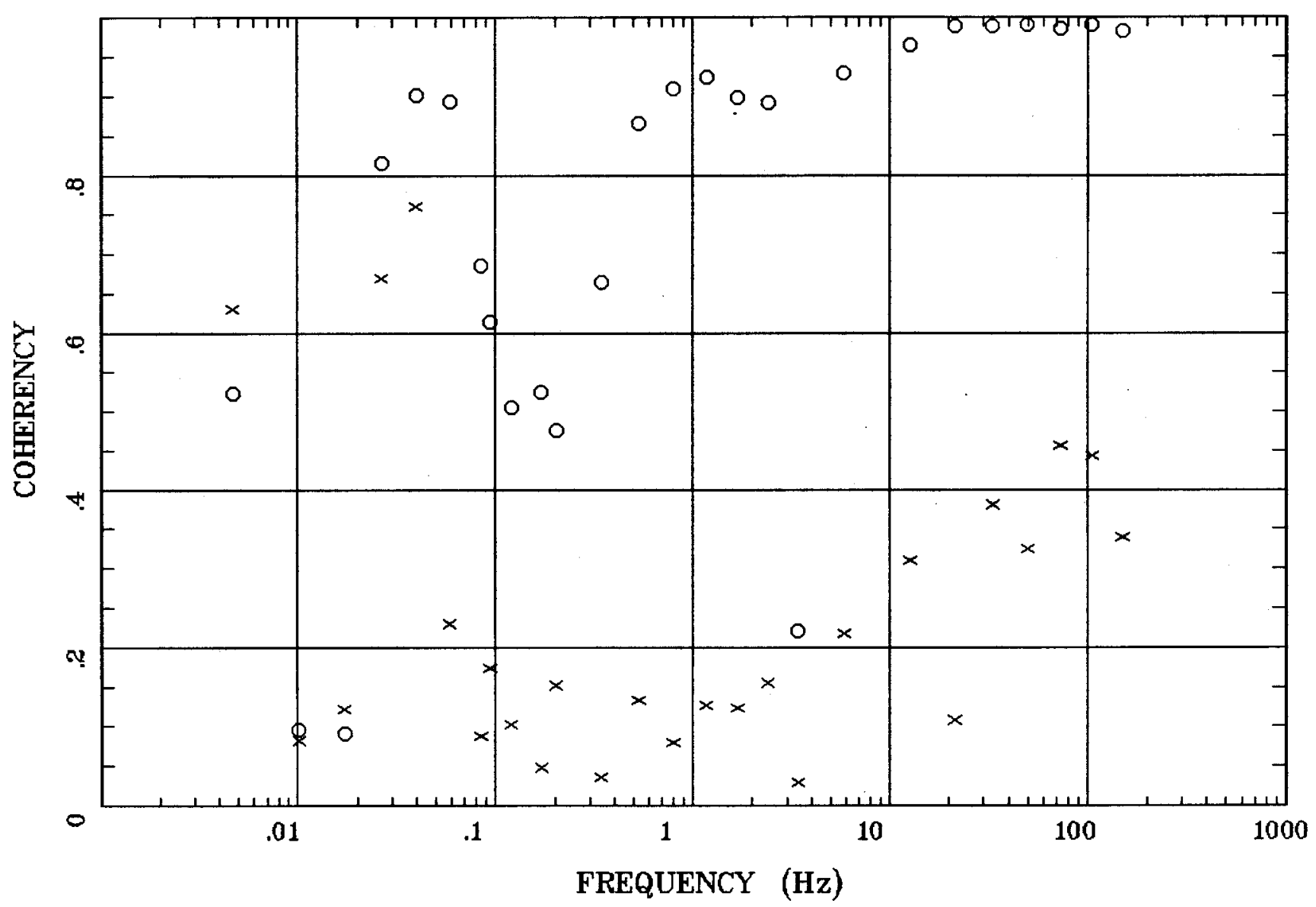

Client:

Remote:

Acquired:

Survey Co:
Rotation:

Filename: NNR06

Channels: Ch1 Ch2 Ch3 Ch4 Ch5 Ch6 Ch7

Plotted: 18:15 Jun 24, 1999

< EMI - ElectroMagnetic Instruments > 
Station 06

POLAR PLOTS

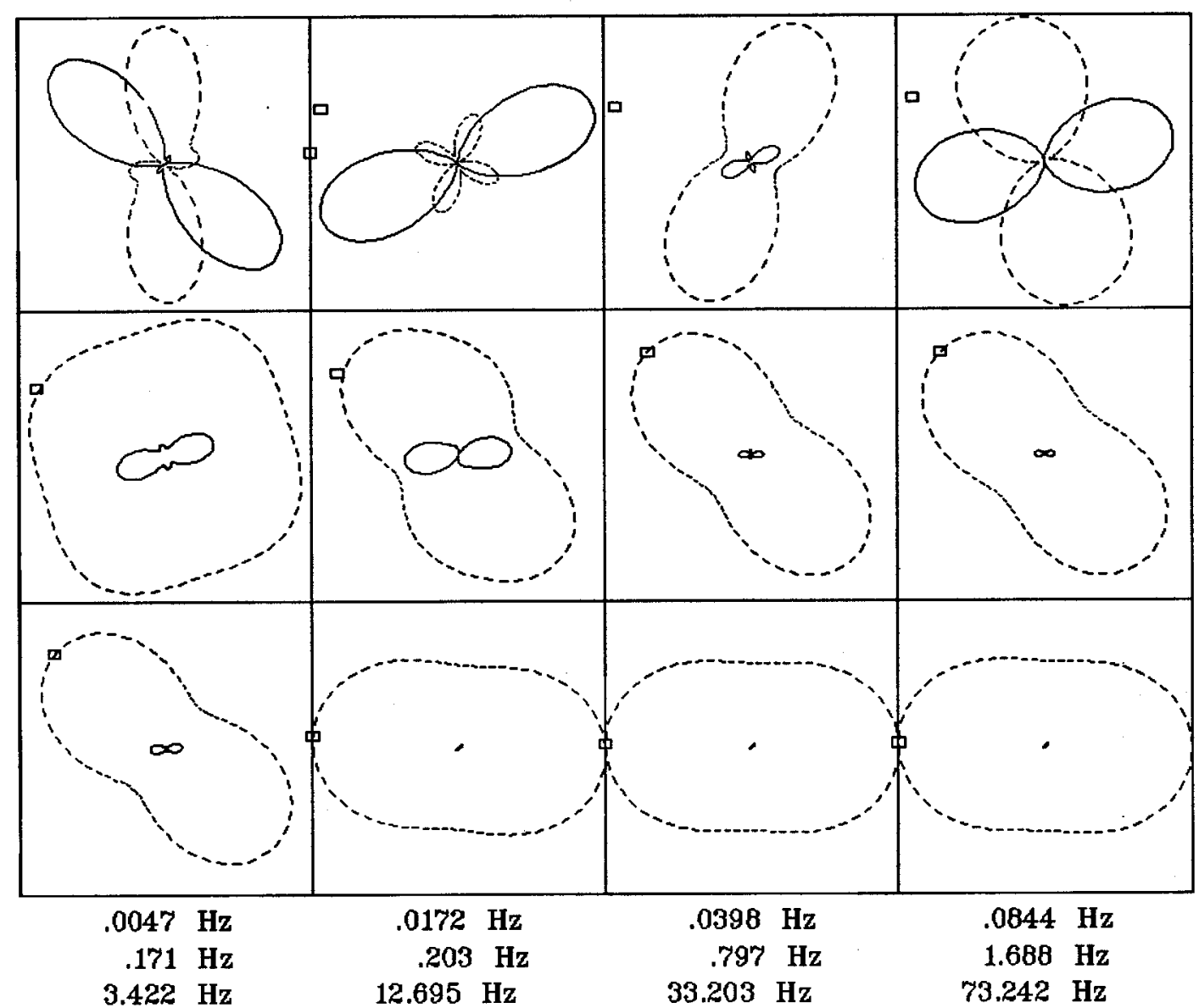

Client:

Remote:

Acquired:

Survey Co:
Rotation:

Filename: NNR06

Channels: Ch1 Ch2 Ch3 Ch4 Ch5 Ch6 Ch7 Plotted: 18:15 Jun 24, 1999

< EMI - ElectroMagnetic Instruments > 


\section{Station 09}

\section{APPARENT RESISTIVITY}

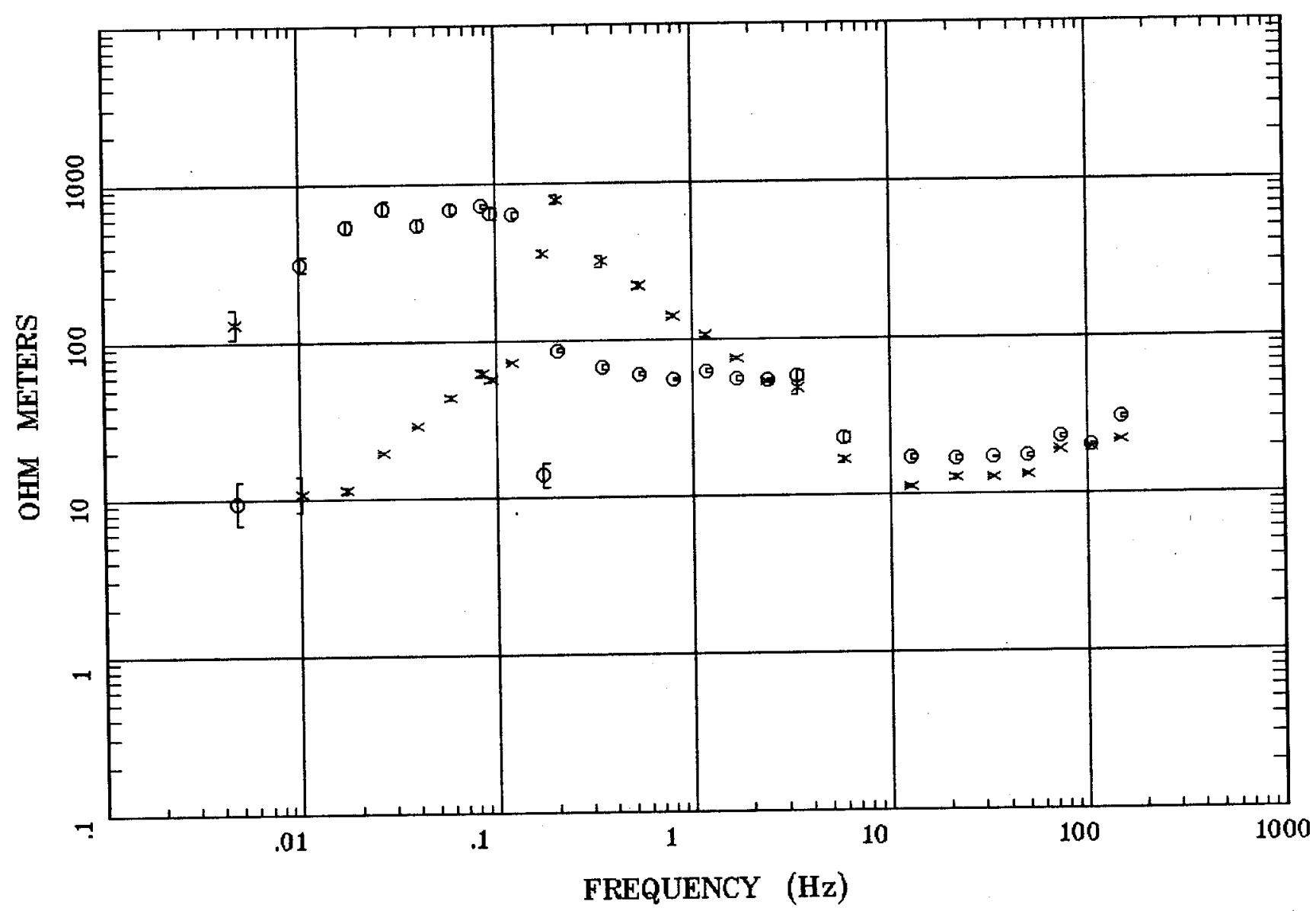

Client:

Rotation

Filename: NNR09

Remote:

Channels: Ch1 Ch2 Ch3 Ch4 Ch5 Ch6 Ch7

Acquired:

Plotted: 17:59 Jun 24, 1999

Survey Co:

< EMI - ElectroMagnetic Instruments > 


\section{Station 09}

\section{IMPEDANCE PHASE}

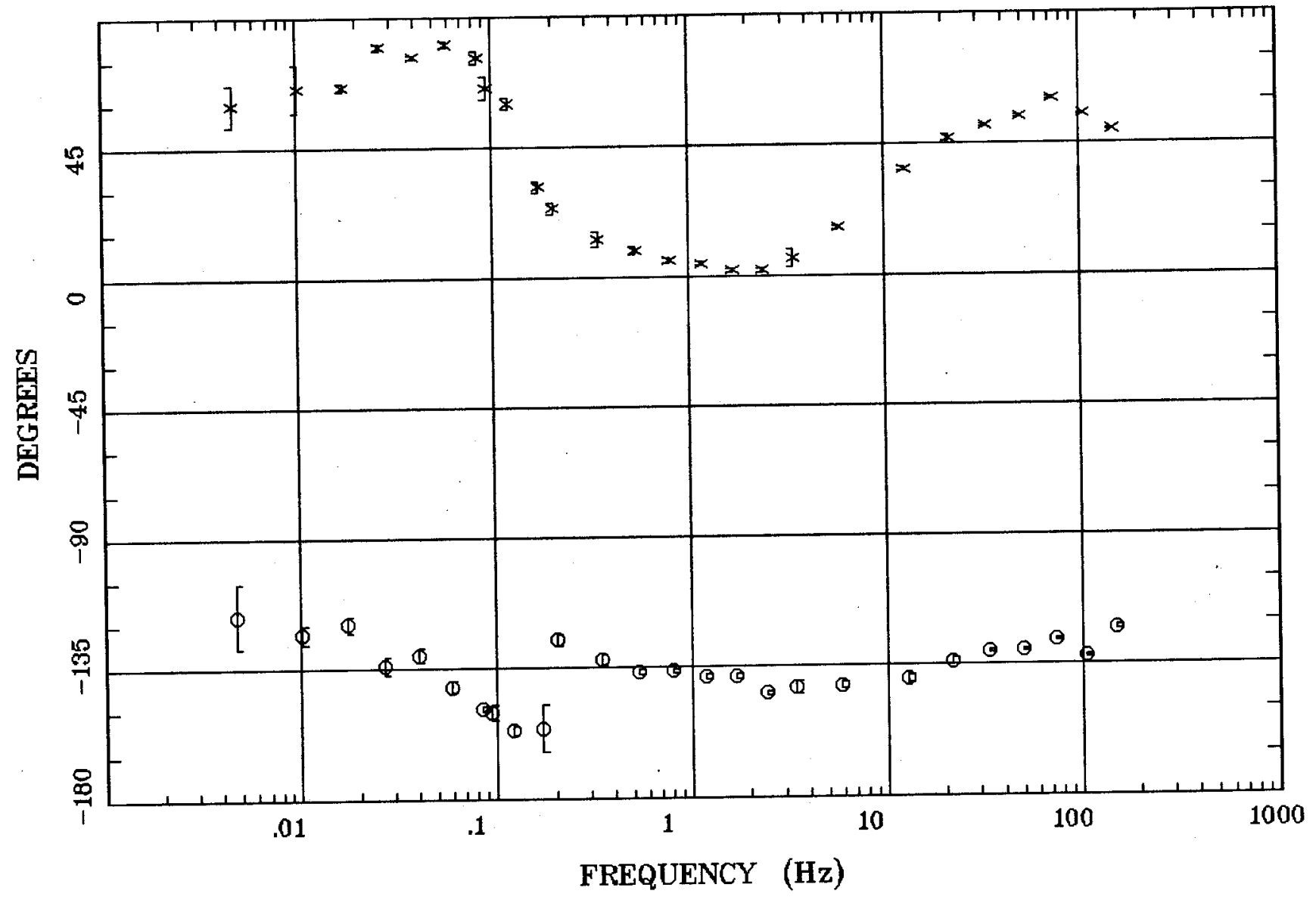

Rotation:

Client:

Remote:

Acquired:

Survey Co:
Filename: NNR09

Channels: Ch1 Ch2 Ch3 Ch4 Ch5 Ch6 Ch7

Plotted: 17:59 Jun 24, 1999

< EMI - ElectroMagnetic Instruments > 


\section{Station 09}

\section{ROTATION ANGLE}

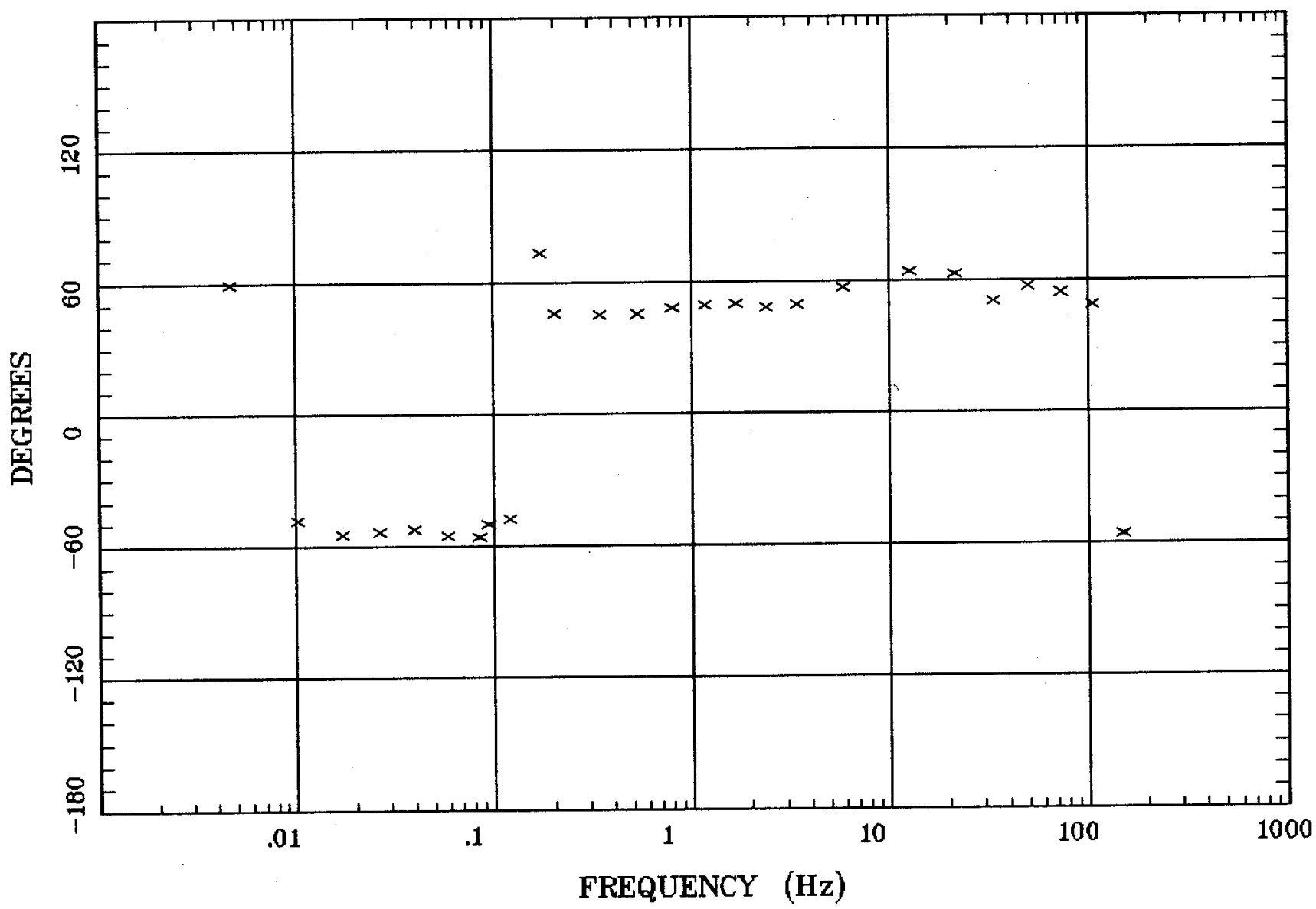

Client:

Remote:

Acquired:

Survey Co:

Rotation:

Filename: NNR09

Channels: Ch1 Ch2 Ch3 Ch4 Ch5 Ch6 Ch7

Plotted: $17: 59$ Jun 24, 1999

< EMI - ElectroMagnetic Instruments > 


\section{Station 09}

\section{IMPEDANCE SKEW}

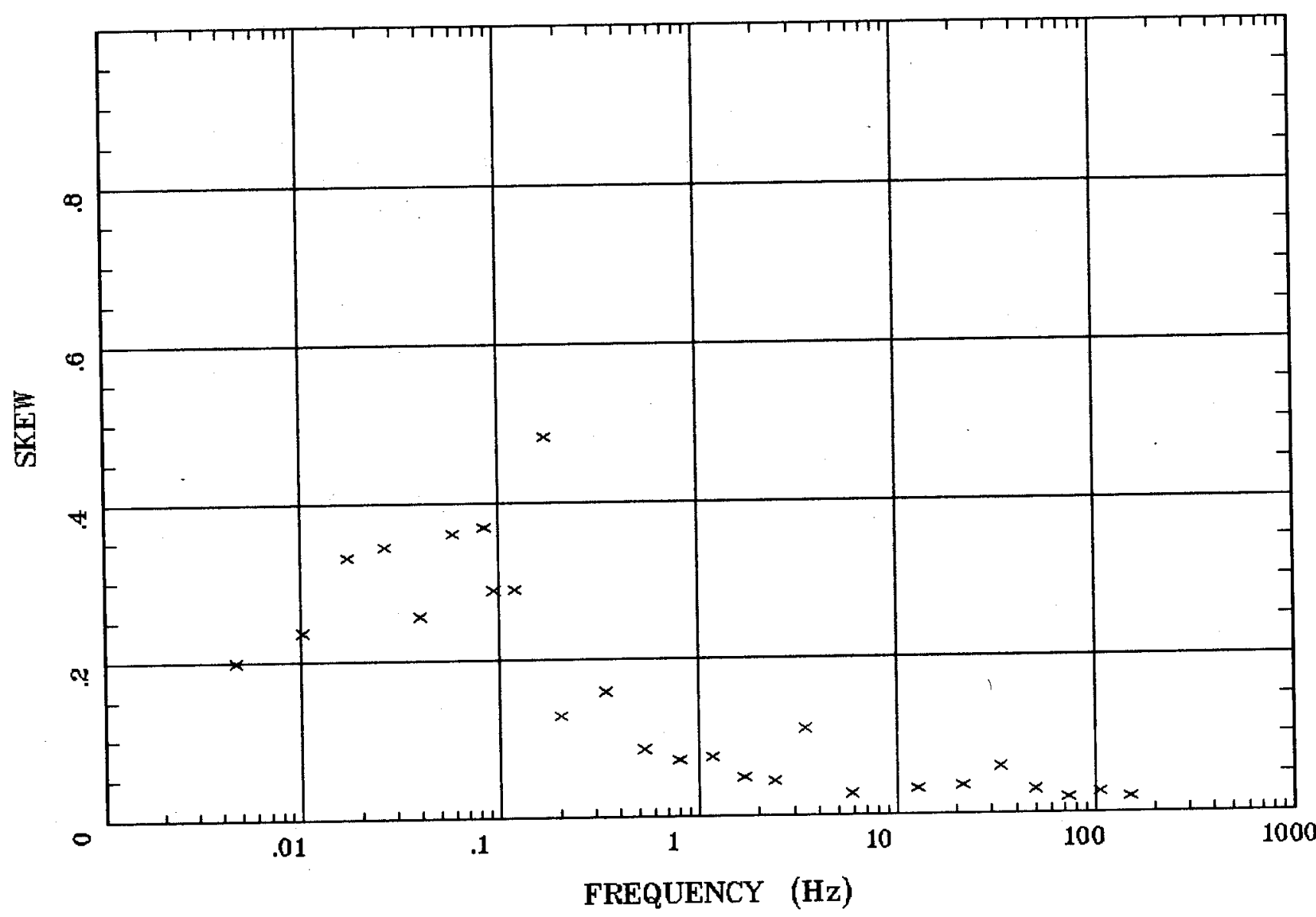

Client:

Remote:

Acquired:

Survey Co:

Rotation:

Filename: NNR09

Channels: Ch1 Ch2 Ch3 Ch4 Ch5 Ch6 Ch7

Plotted: 17:59 Jun 24, 1999

< EMI - ElectroMagnetic Instruments 


\section{Station 09}

E MULT Coh.

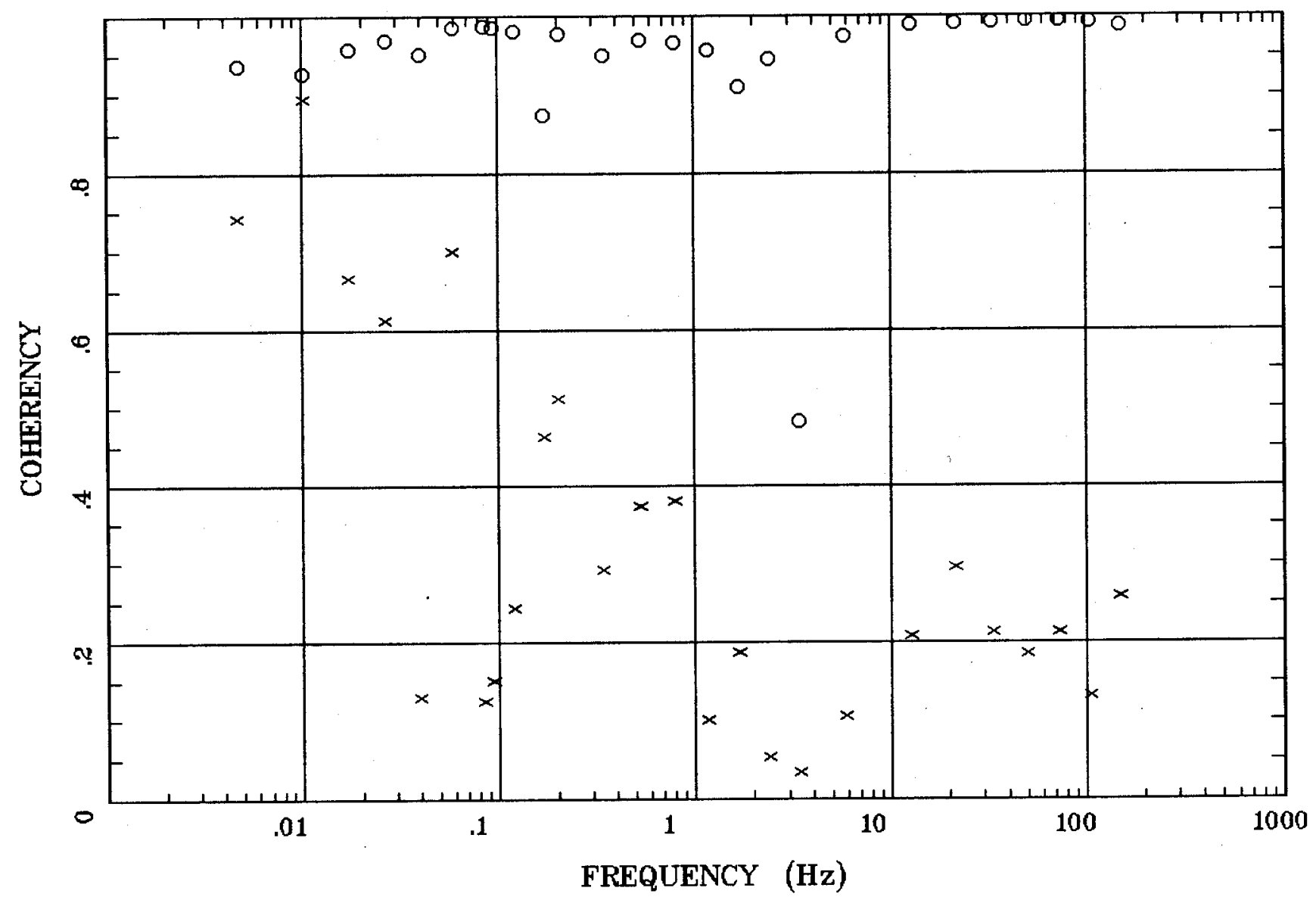

Client:

Rotation:

Remote:

Filename: NNR09

Acquired:

Channels: Ch1 Ch2 Ch3 Ch4 Ch5 Ch6 Ch7

Plotted: 17:59 Jun 24, 1999

Survey Co:

< EMI - ElectroMagnetic Instruments > 


\section{POLAR PLOTS}

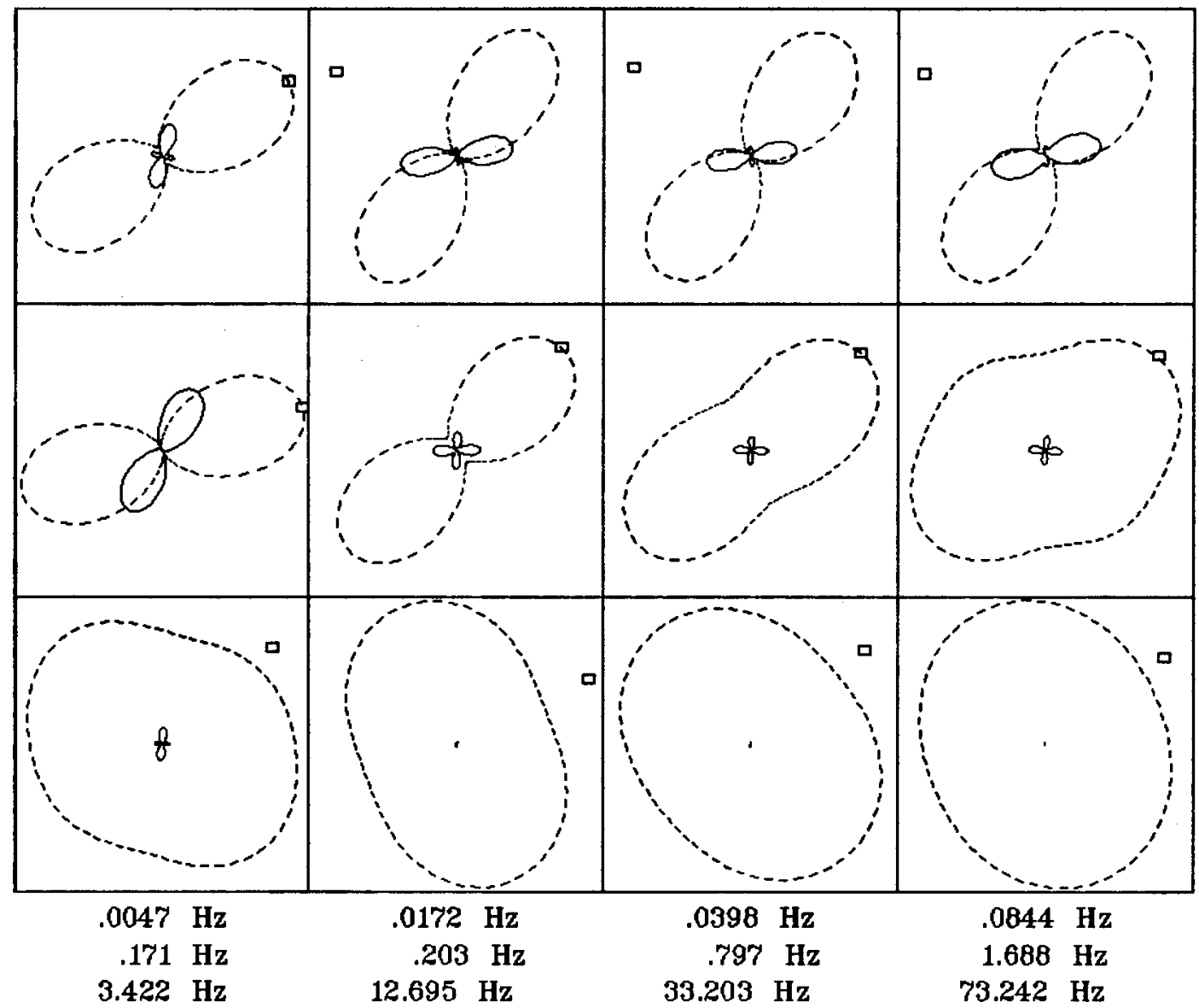

Client:

Remote:

Acquired:

Survey Co:
Rotation:

Filename: NNR09

Channels: Ch1 Ch2 Ch3 Ch4 Ch5 Ch6 Ch7

Plotted: 17:59 Jun 24, 1999

< EMI - ElectroMagnetic Instruments 


\section{Station 08}

\section{APPARENT RESISTIVITY}

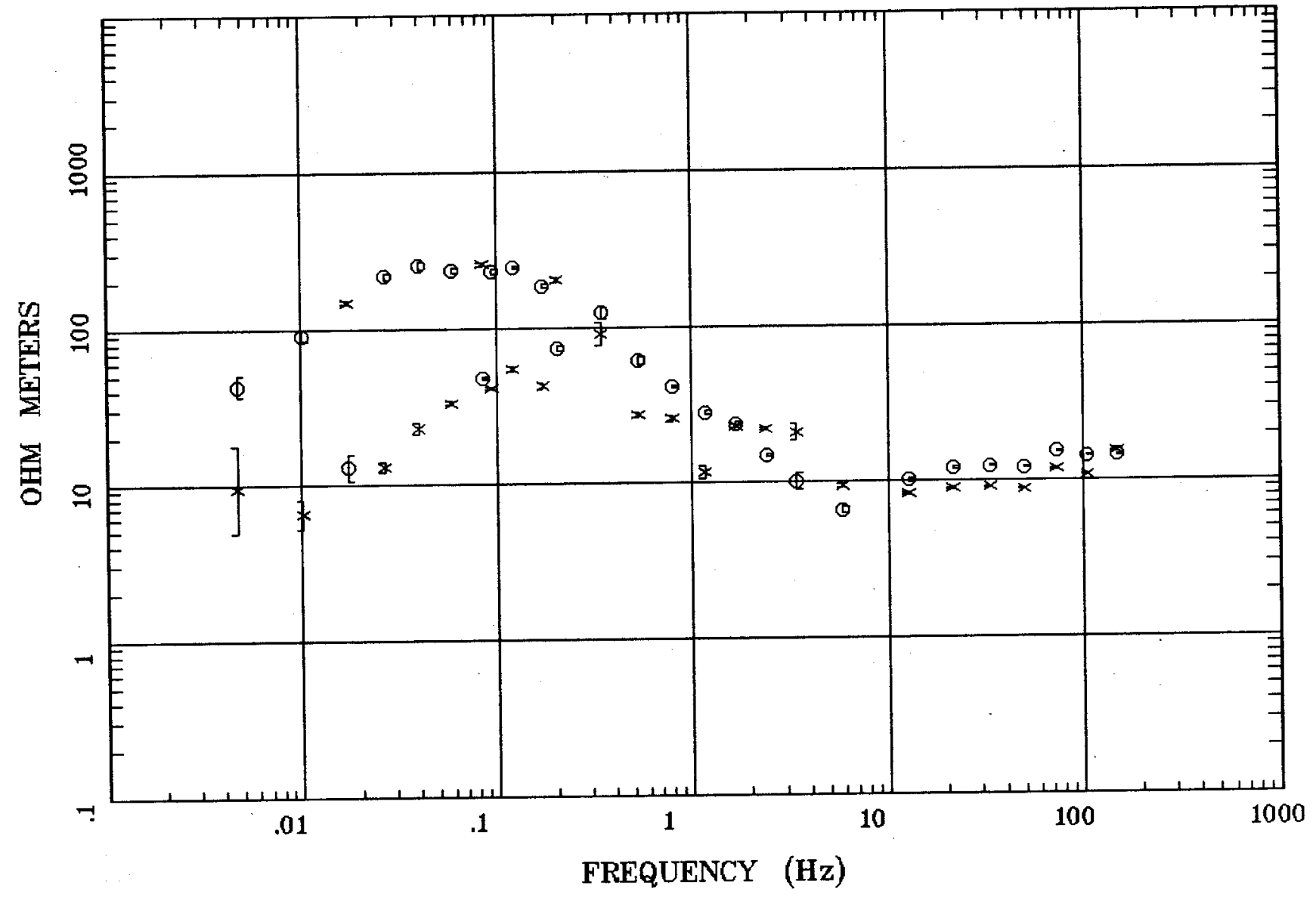

Rotation:

Client:

Filename: NNROB

Remote:

Channels: Ch1 Ch2 Ch3 Ch4 Ch5 Ch6 Chr

Plotted: 18:07 Jun 24, 1999

Acquired:

Survey Co:

< EMI - ElectroMagnetic Instruments > 
Station 08

\section{IMPEDANCE PHASE}

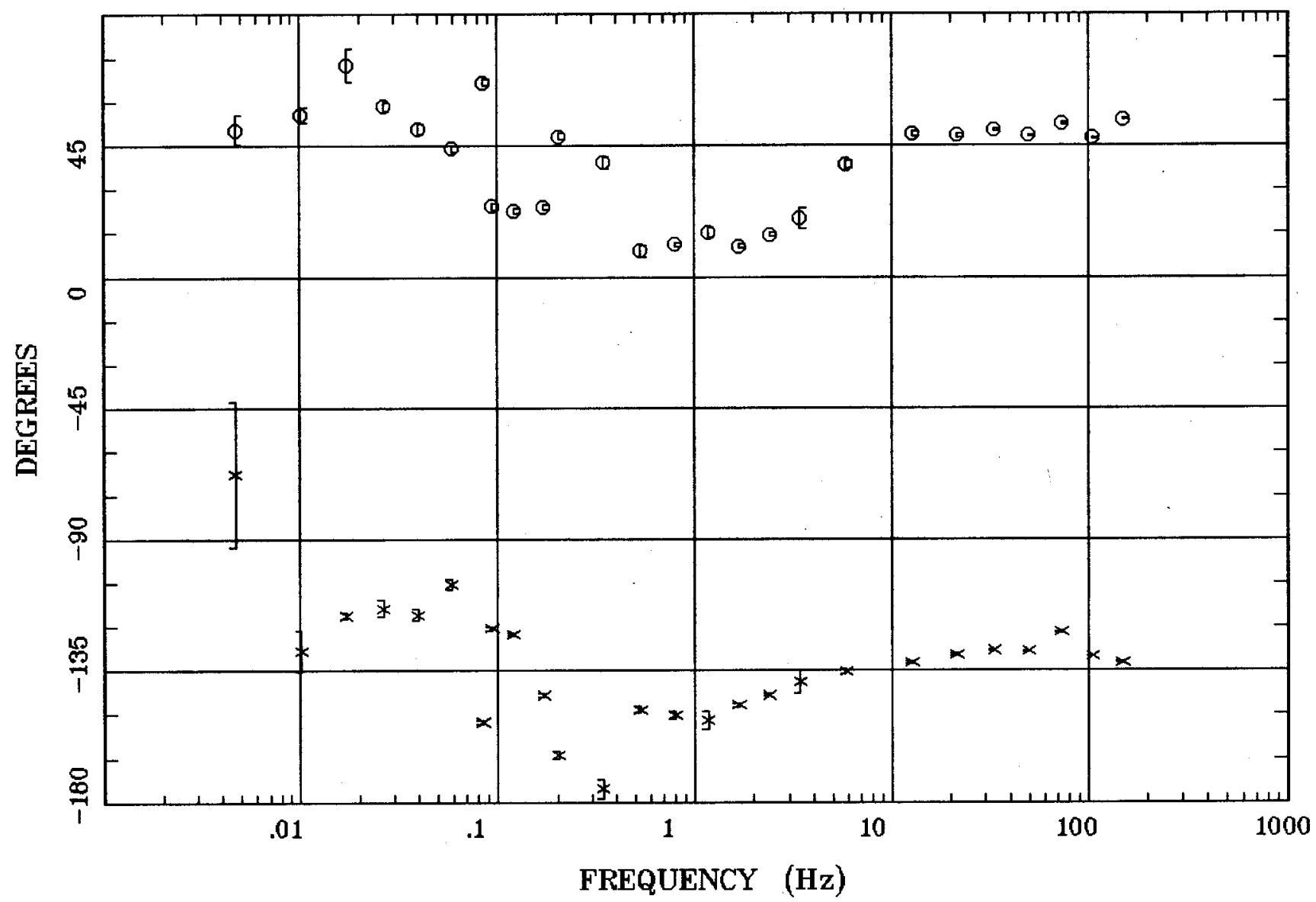

Client:

Rotation:

\section{Remote:}

Acquired:

Filename: NNR08

Channels: Ch1 Ch2 Ch3 Ch4 Ch5 Ch6 Ch7

Survey Co:

Platted: 18:07 Jun 24, 1999

< EMI - ElectroMagnetic Instruments 
Station 08

\section{ROTATION ANGLE}

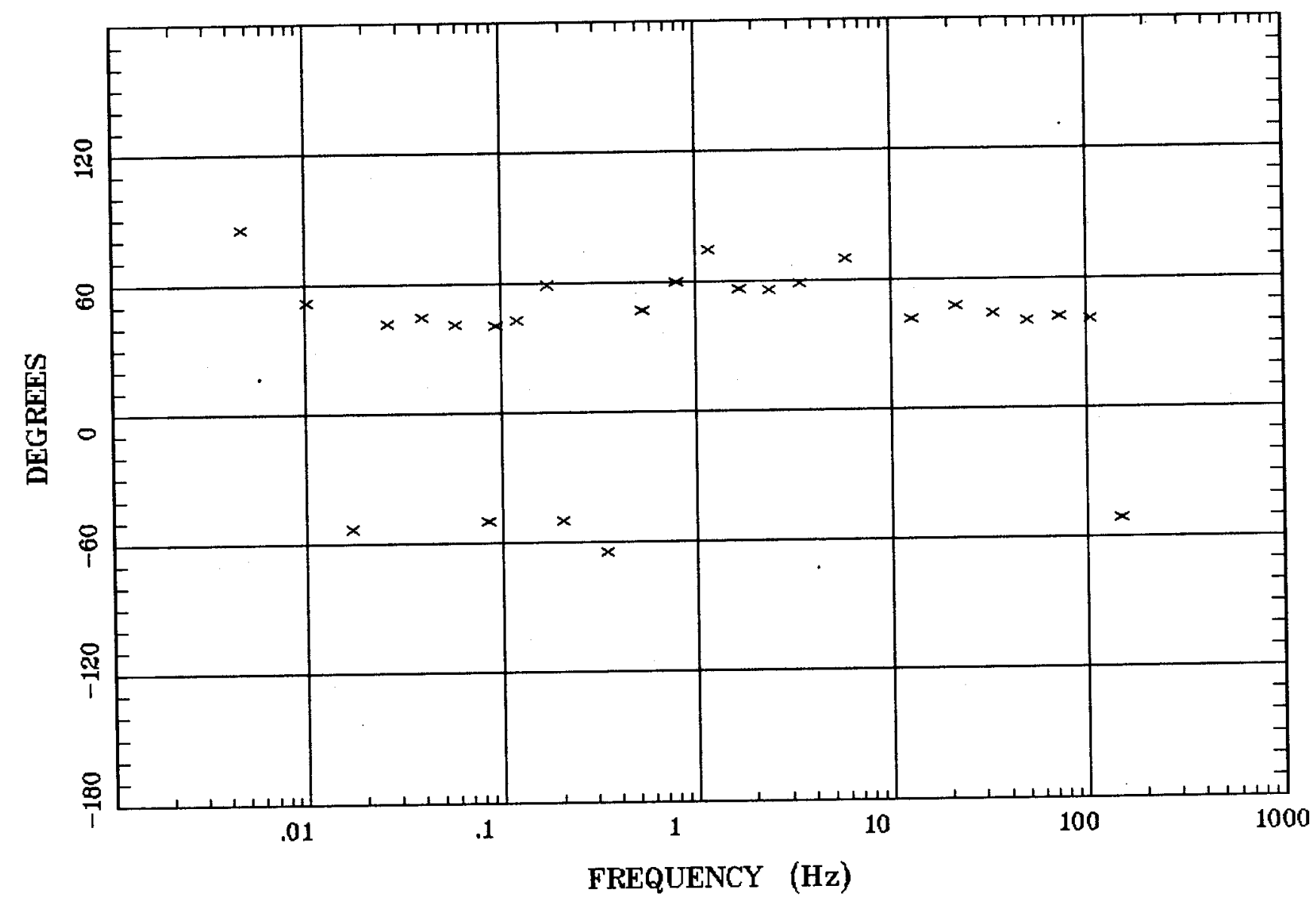

Client:

Rotation:

Filename: NNROB

mote.

Channels: Ch1 Ch2 Ch3 Ch4 Ch5 Ch6 Ch7

Acquired:

Survey Co:

Plotted: 18:07 Jun 24, 1999

$<$ EMI - ElectroMagnetic Instruments > 


\section{IMPEDANCE SKEW}

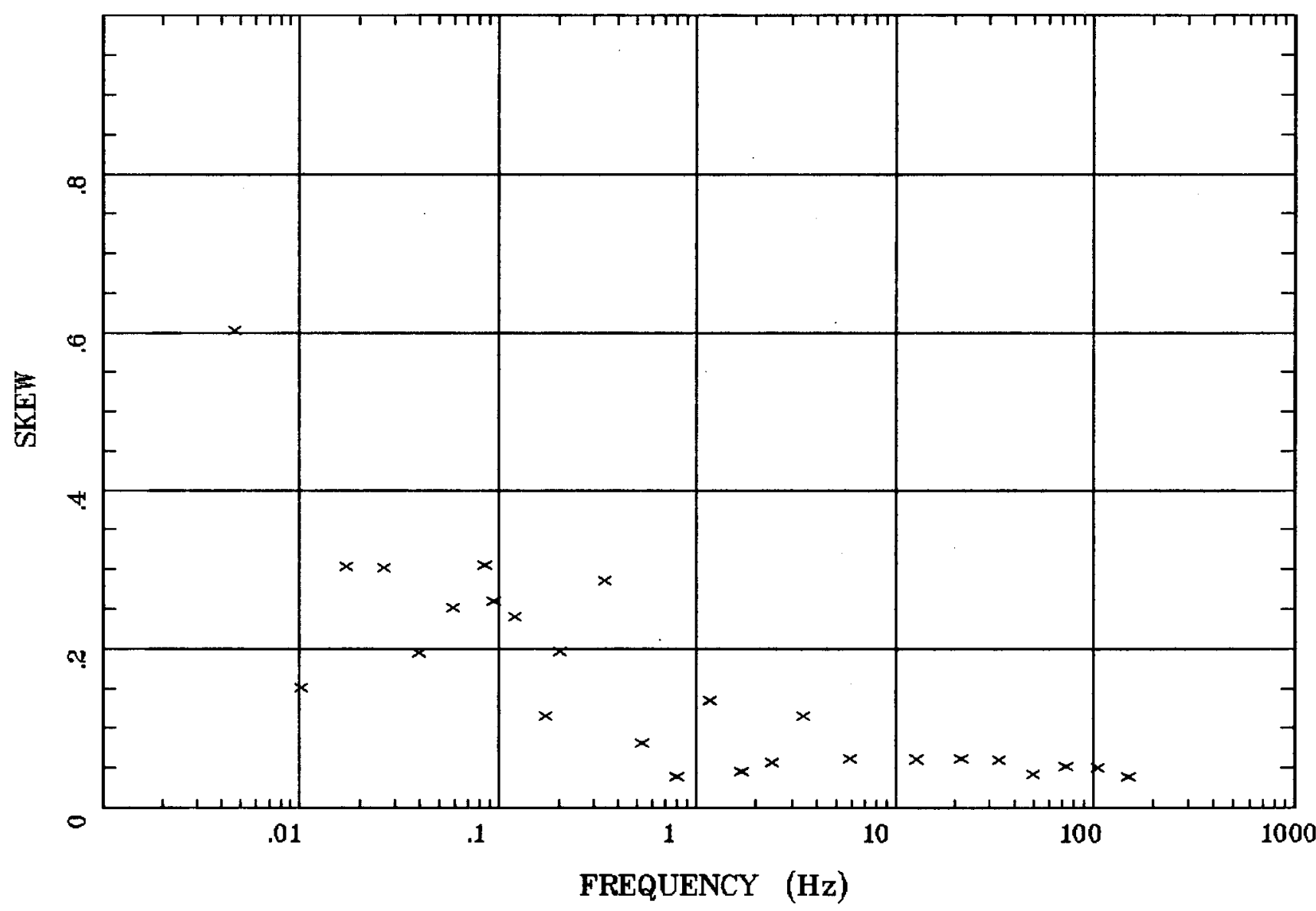

Client:

Remote:

Acquired:

Survey Co:
Rotation:

Filename: NNR0B

Channels: Ch1 Ch2 Ch3 Ch4 Ch5 Ch6 Chry

Plotted: 18:07 Jun 24, 1999

$<$ EMI - ElectroMagnetic Instruments 
Station 08

E MULT Coh.

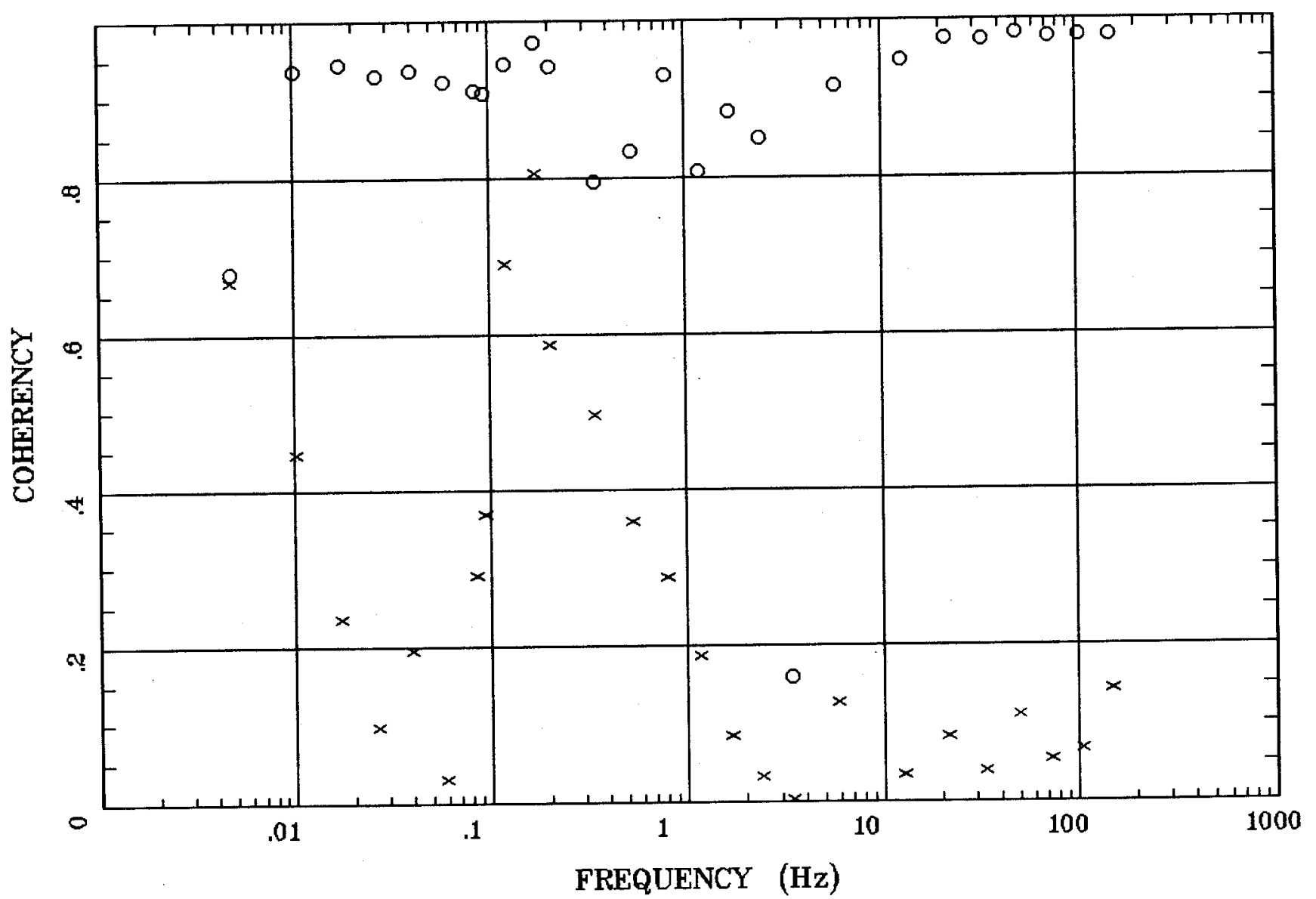

Client:

Remote:

Acquired:

Survey Co:
Rotation:

Filename: NNR0B

Channels: Ch1 Ch2 Ch3 Ch4 Ch5 Ch6 Ch7 Plotted: 18:07 Jun 24, 1999

< EMI - ElectroMagnetic Instruments > 


\section{POLAR PLOTS}

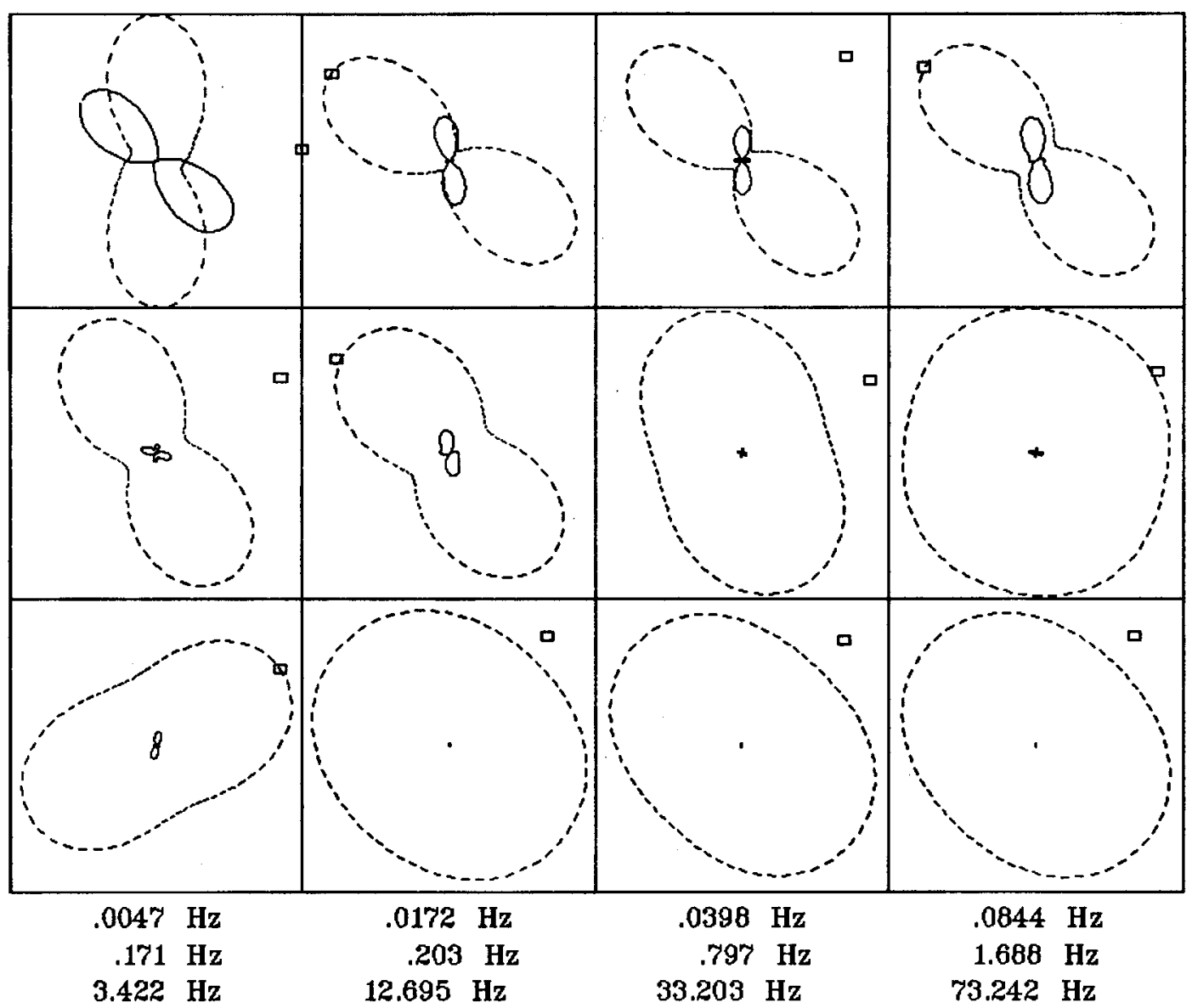

Client:

Remote:

Acquired:

Survey Co:
Rotation:

Filename: NNR08

Channels: Ch1 Ch2 Ch3 Ch4 Ch5 Ch6 Ch7

Plotted: 18:07 Jun 24, 1999

< EMI - ElectroMagnetic Instruments > 
Station 07

APPARENT RESISTIVITY

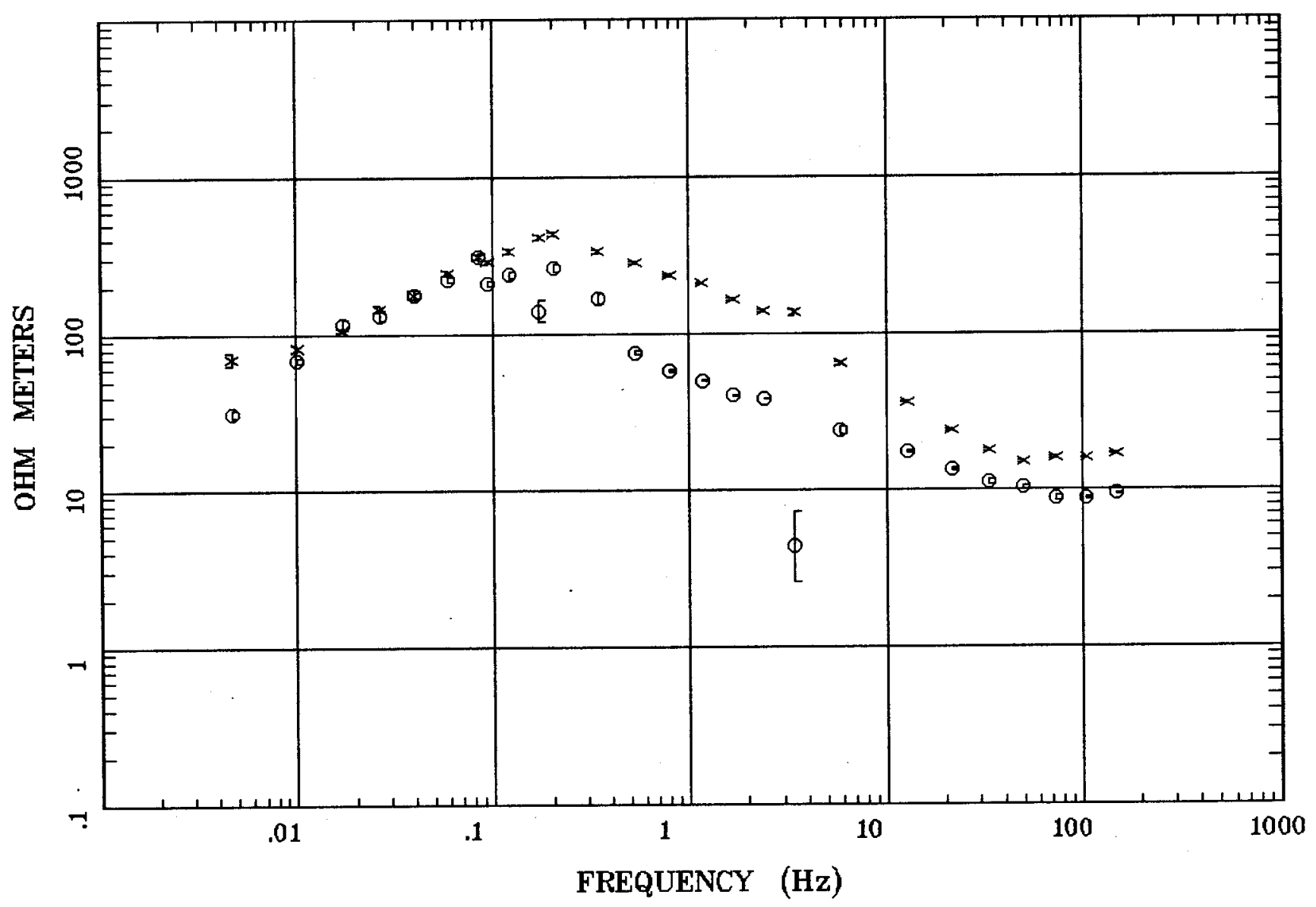

Client:

Remote:

Acquired:

Survey Co:
Rotation:

Filename: NNRO

Channels: Ch1 Ch2 Ch3 Ch4 Ch5 Ch6 Ch7

Plotted: 18:13 Jun 24, 1999

< EMI - ElectroMagnetic Instruments > 


\section{Station 07}

\section{IMPEDANCE PHASE}

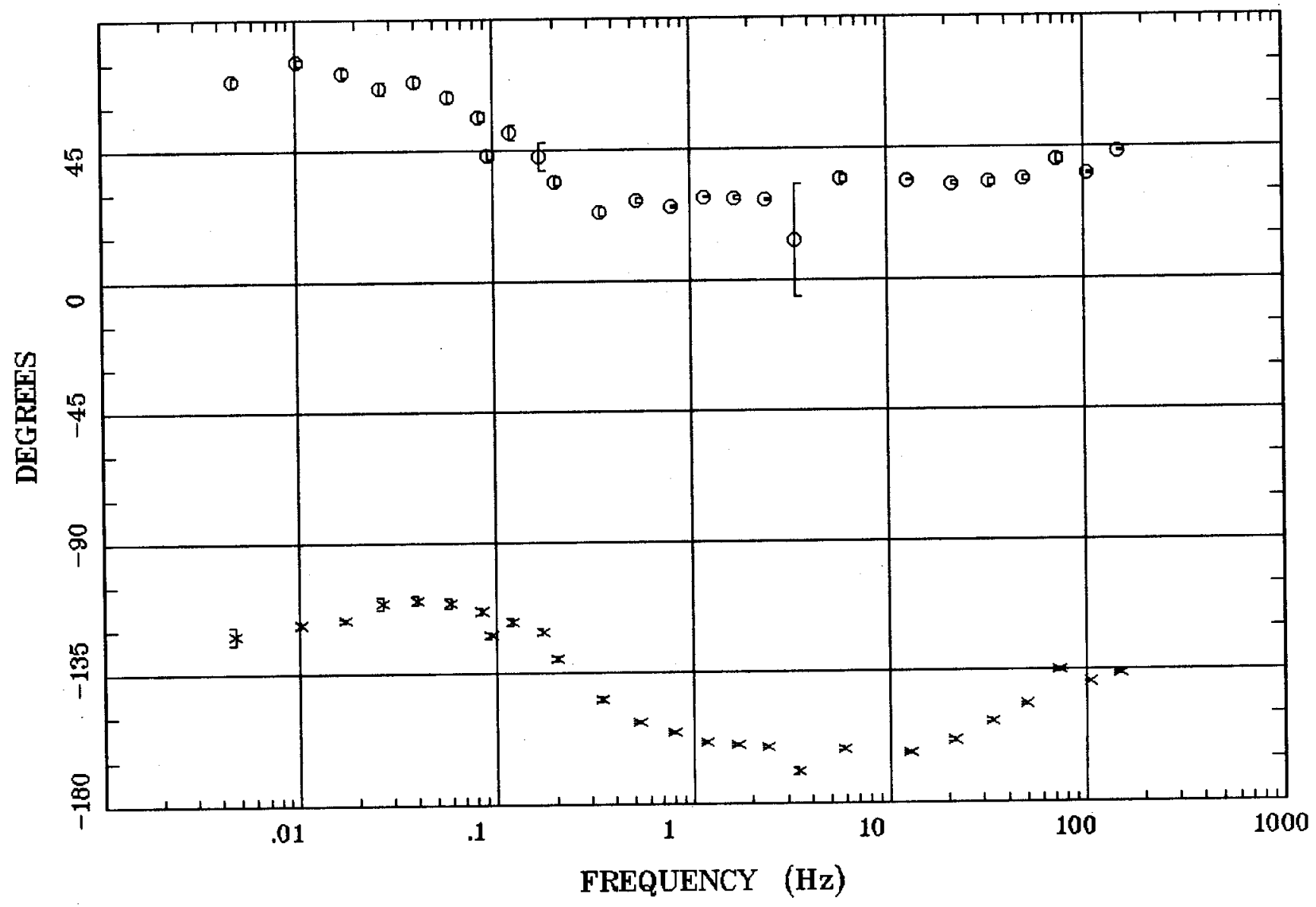

Client:

Rotation:

Remote:

Filename: NNR07

Channels: Ch1 Ch2 Ch3 Ch4 Ch5 Ch6 Ch'7

Acquired:

Plotted: 18:13 Jun 24, 1999

Survey Co:

< EMI - ElectroMagnetic Instruments 


\section{Station 07}

\section{ROTATION ANGLE}

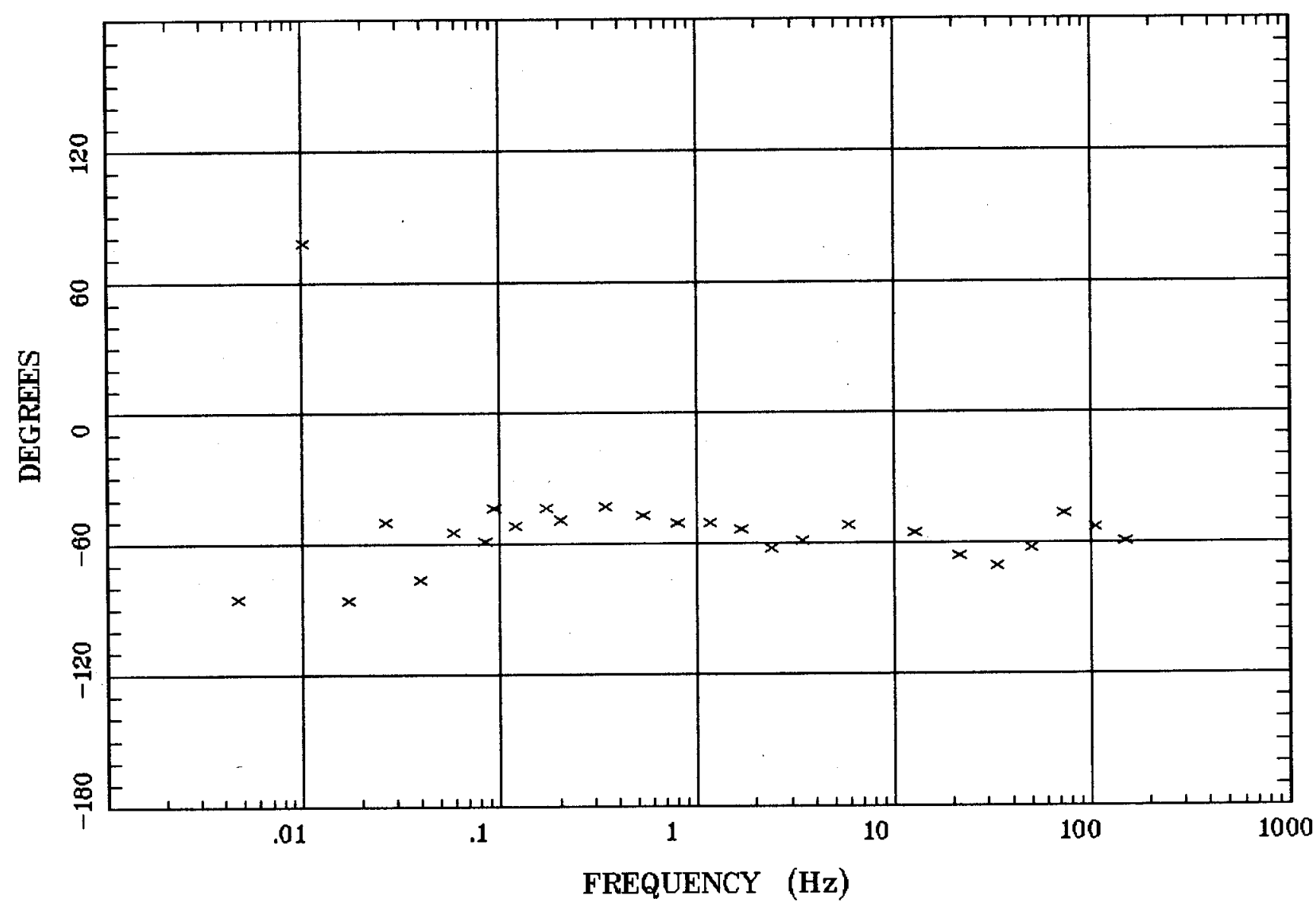

Client:

Rotation:

Remote:

Filename: NNRO?

Acquired:

Channels: Ch1 Ch2 Ch3 Ch4 Ch5 Ch6 Ch7

Plotted: 18:13 Jun 24, 1999

Survey Co:

< EMI - ElectroMagnetic Instruments 


\section{Station 07}

\section{IMPEDANCE SKEW}

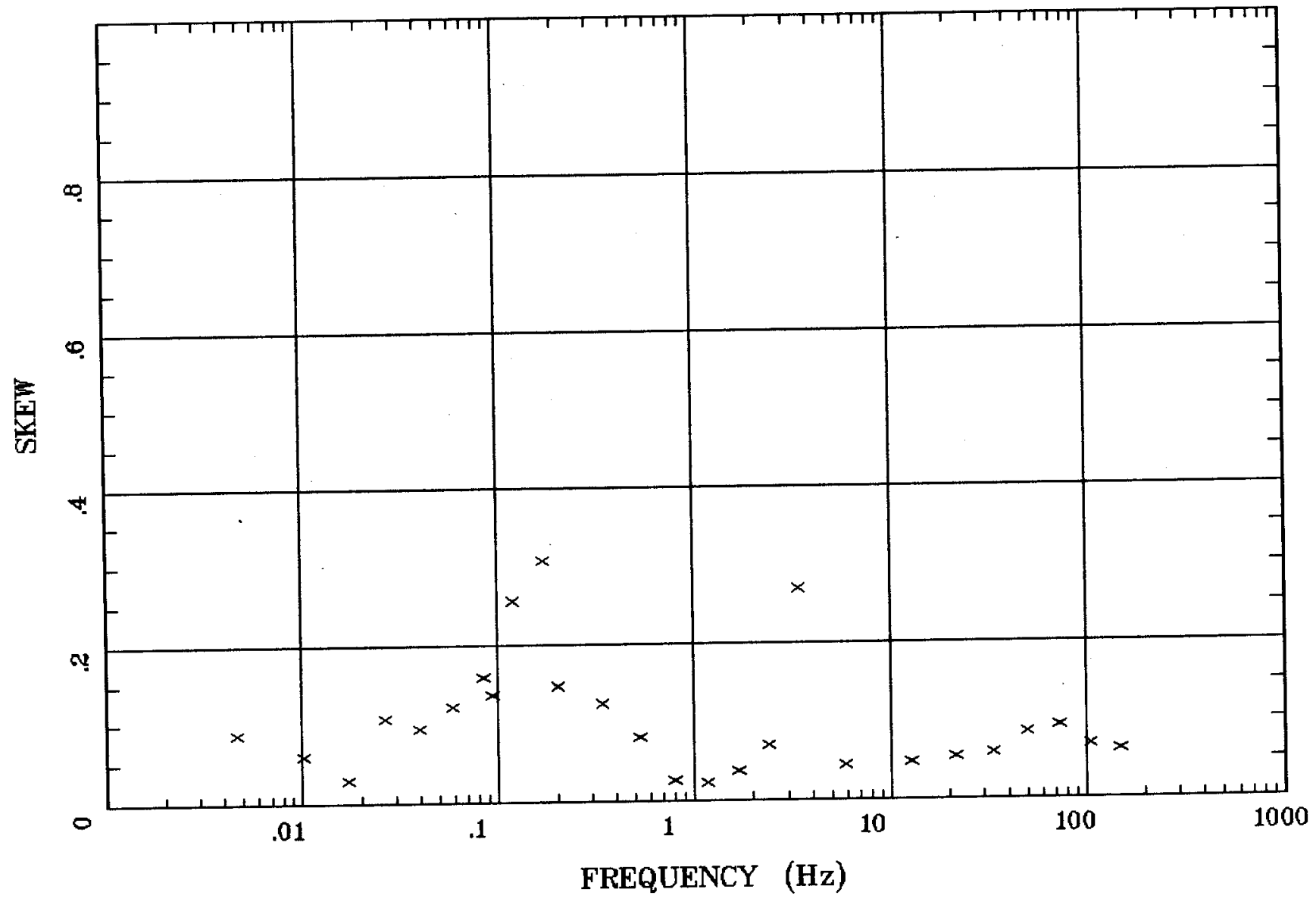

Client:

Rotation:

Filename: NNR07

Acquired:

Channels: Ch1 Ch2 Ch3 Ch4 Ch5 Ch6 Ch'

Survey Ca:

Plotted: 18:13 Jun 24, 1999

< EMI - ElectroMagnetic Instruments > 


\section{Station 07}

E MULT Coh.

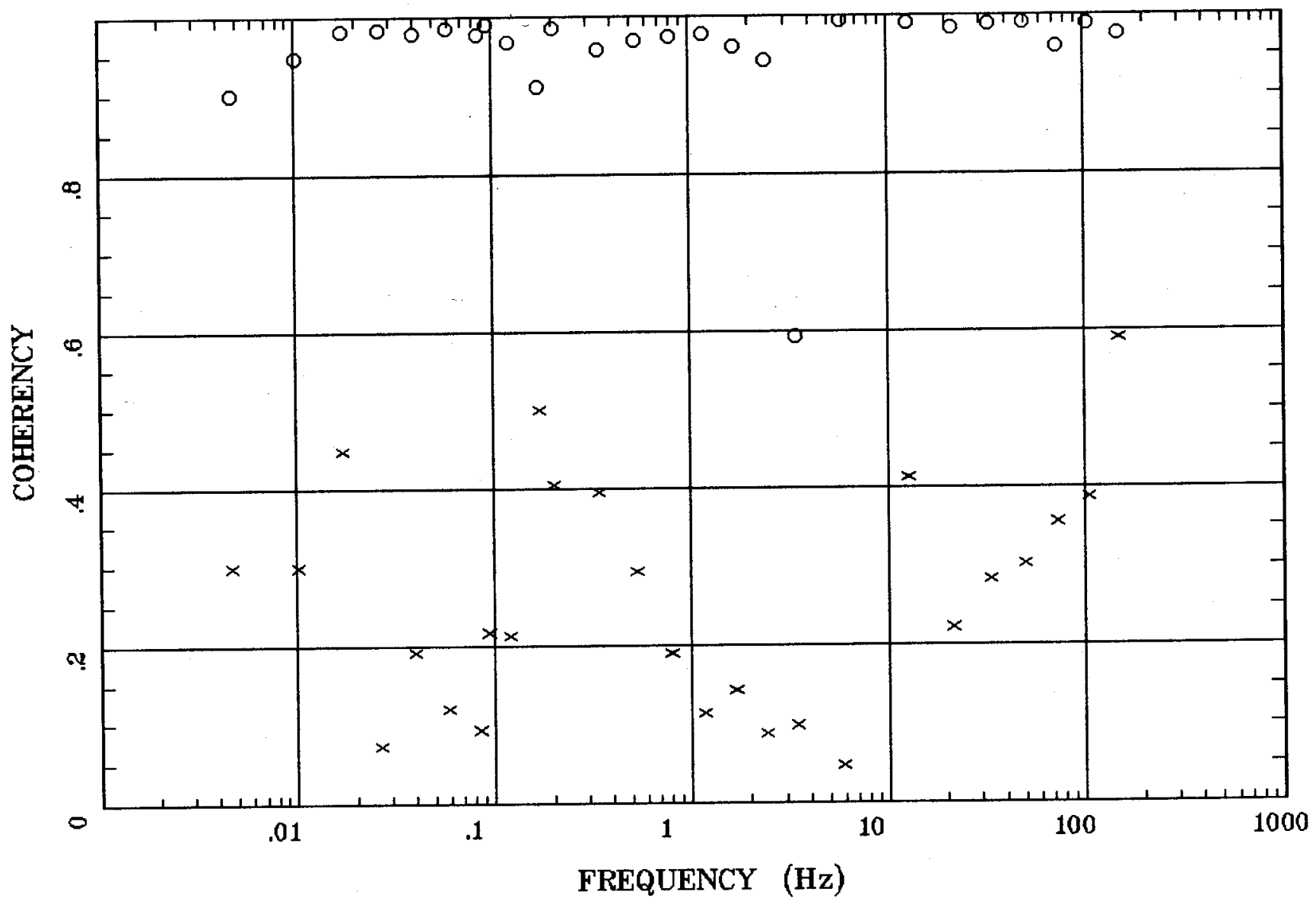

Client:

Rotation:

Remote:

Filename: NNR07

Acquired:

Channels: Ch1 Ch2 Ch3 Ch4 Ch5 Ch6 Ch7 Survey Co:

Plotted: 18:13 Jun 24, 1999

< EMI - ElectroMagnetic Instruments > 


\section{Station 07}

\section{POLAR PLOTS}

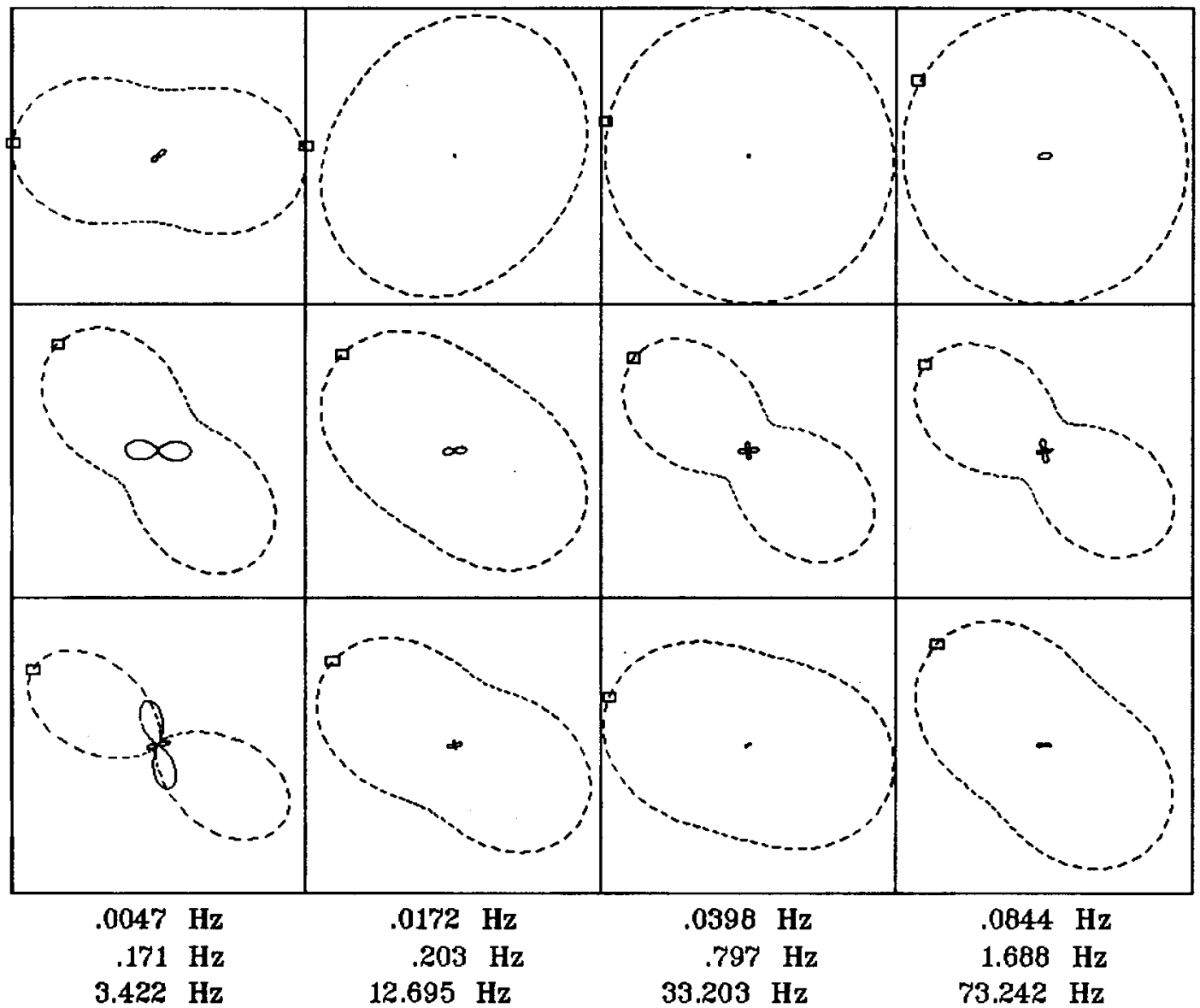

Client:

Remote:

Acquired:

Survey Co:
Rotation:

Filename: NNRO?

Channels: Ch1 Ch2 Ch3 Ch4 Ch5 Ch6 Ch7

Plotted: 18:13 Jun 24, 1999

< EMI - ElectroMagnetic Instruments > 


\section{APPARENT RESISTIVITY}

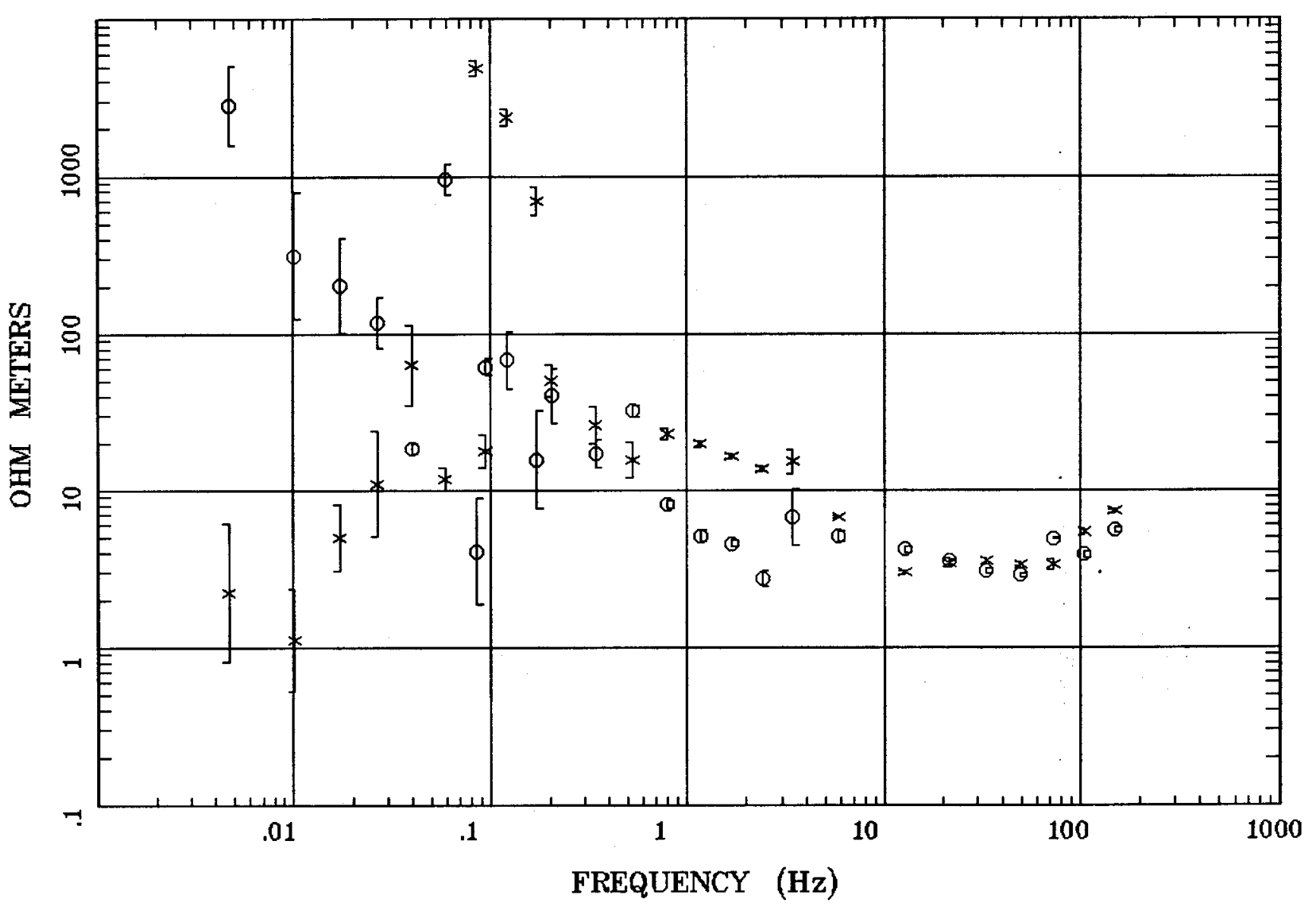

Client:

Remote:

Acquired:

Survey Co:
Rotation:

Filename: CT18

Channels: Ch1 Ch2 Ch3 Ch4 Ch5 Ch6 Ch7

Plotted: 10:14 Jun 25, 1999

< EMI - ElectroMagnetic Instruments > 


\section{Station 18}

\section{IMPEDANCE PHASE}

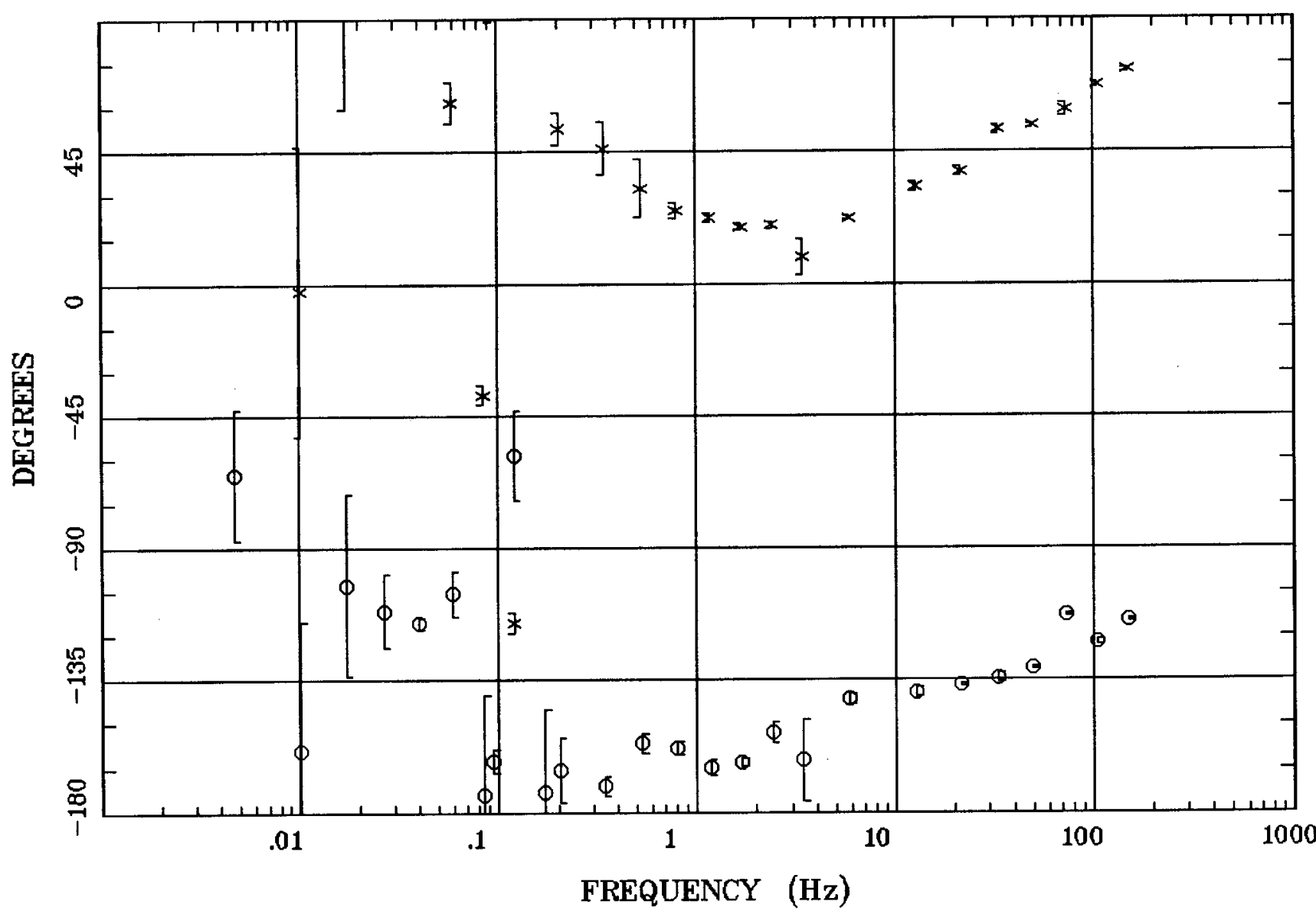

Client:

Rotation:

Remote:

Filename: CT18

Acquired:

Channels: Ch1 Ch2 Ch3 Ch4 Ch5 Ch6 Ch7 Survey Co:

Plotted: 10:14 Jun 25, 1999

< EMI - ElectroMagnetic Instruments > 


\section{ROTATION ANGLE}

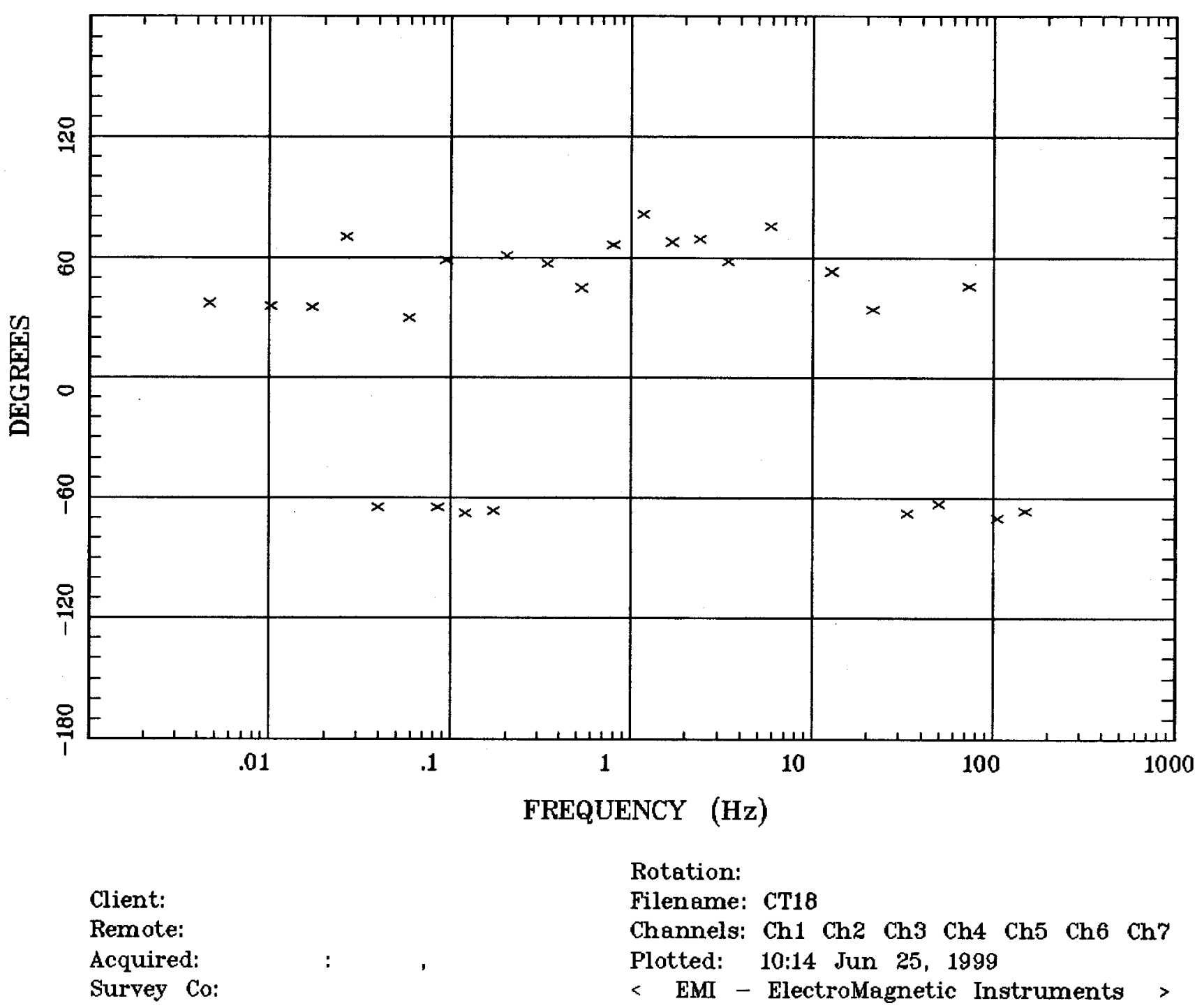




\section{Station 18}

IMPEDANCE SKEW

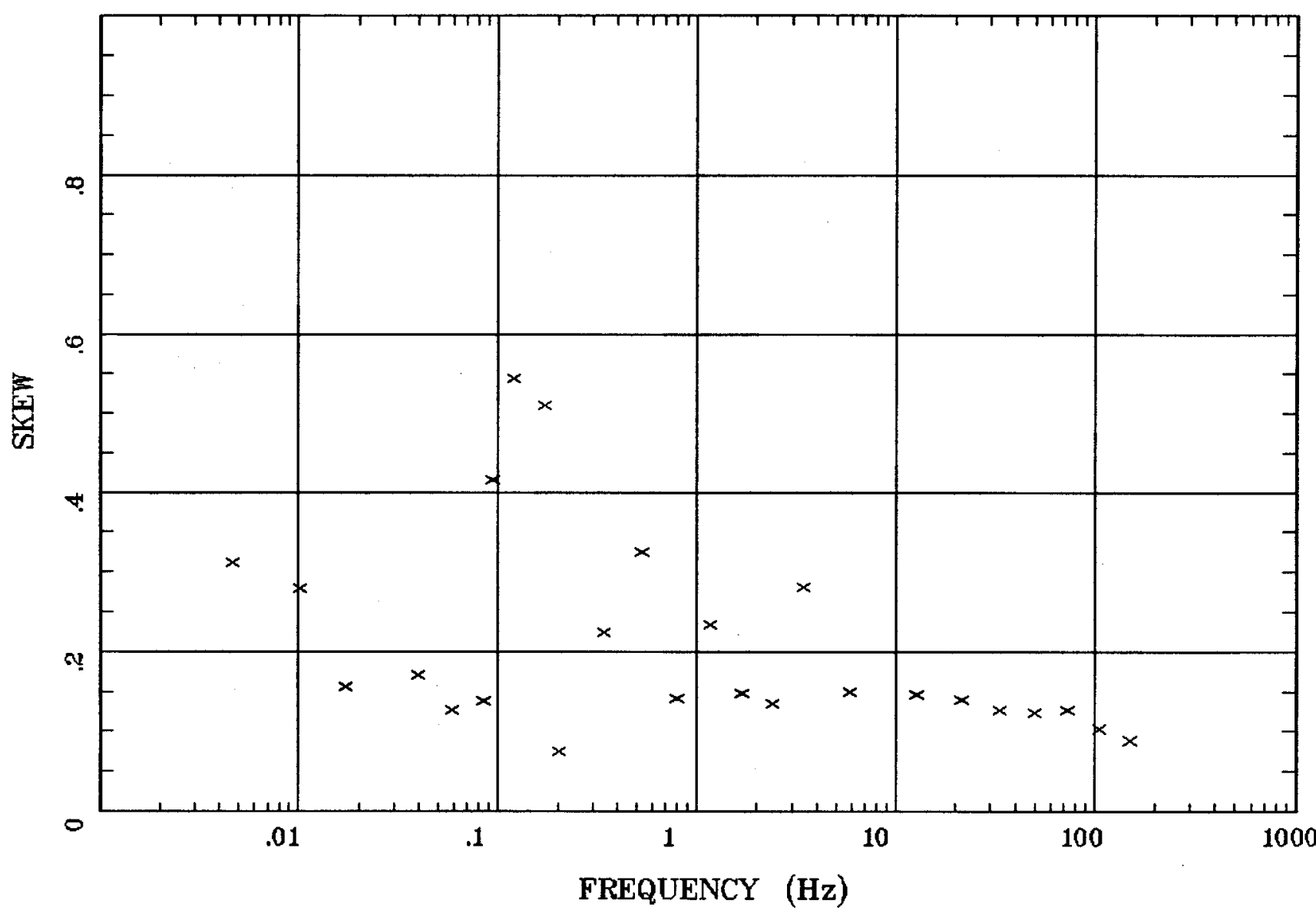

Client:

Remote:

Acquired:

Survey Co:
Rotation:

Filename: CT18

Channels: Ch1 Ch2 Ch3 Ch4 Ch5 Ch6 Ch7

Plotted: 10:14 Jun 25, 1999

$<$ EMI - ElectroMagnetic Instruments 
Station 18

E MULT Coh.

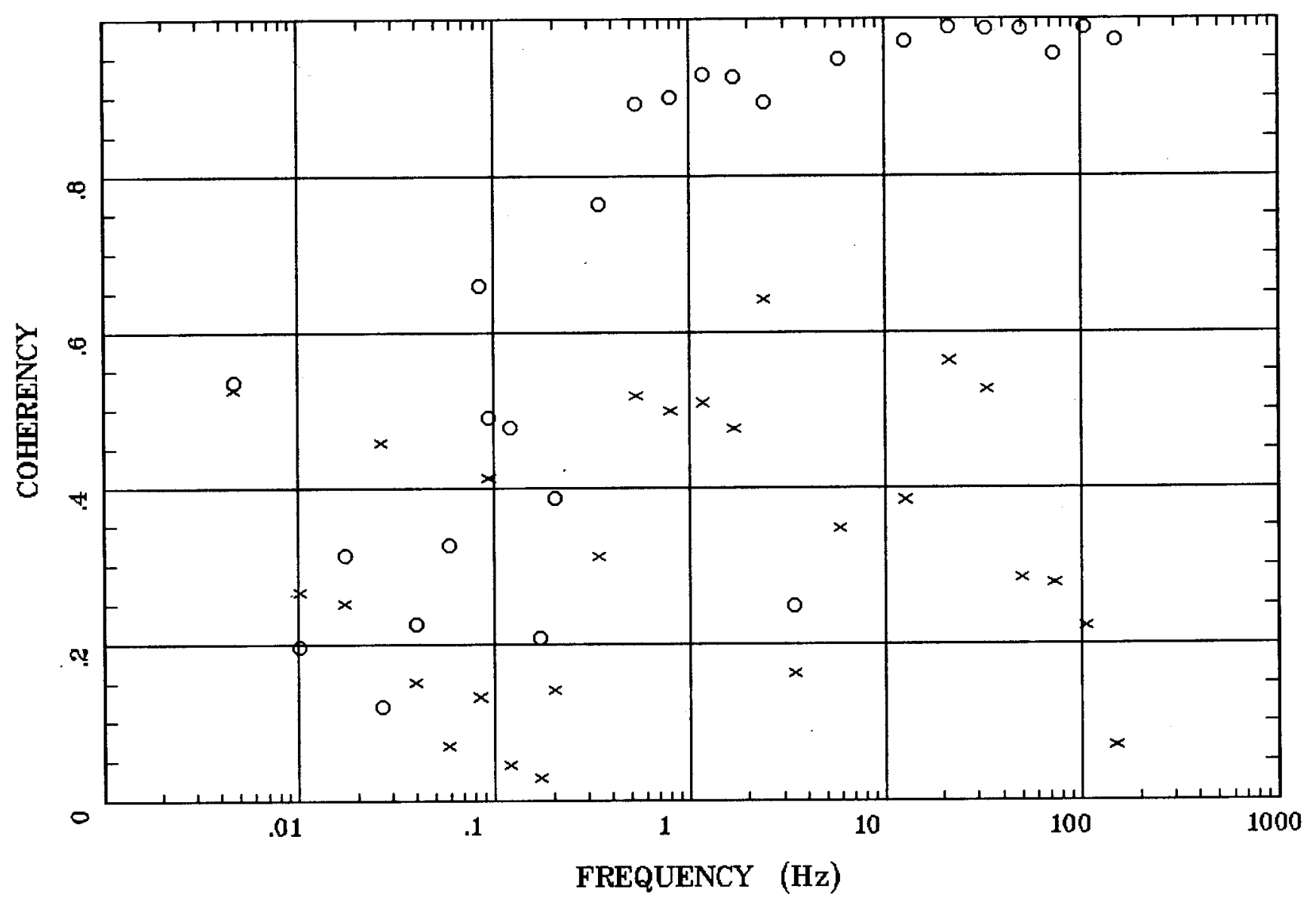

Client:

Rotation:

Filename: CT18

Remote:

Channels: Ch1 Ch2 Ch3 Ch4 Ch5 Ch6 Ch7

Acquired:

Plotted: 10:14 Jun 25, 1999

Survey Ca:

< EMI - ElectroMagnetic Instruments > 


\section{POLAR PLOTS}

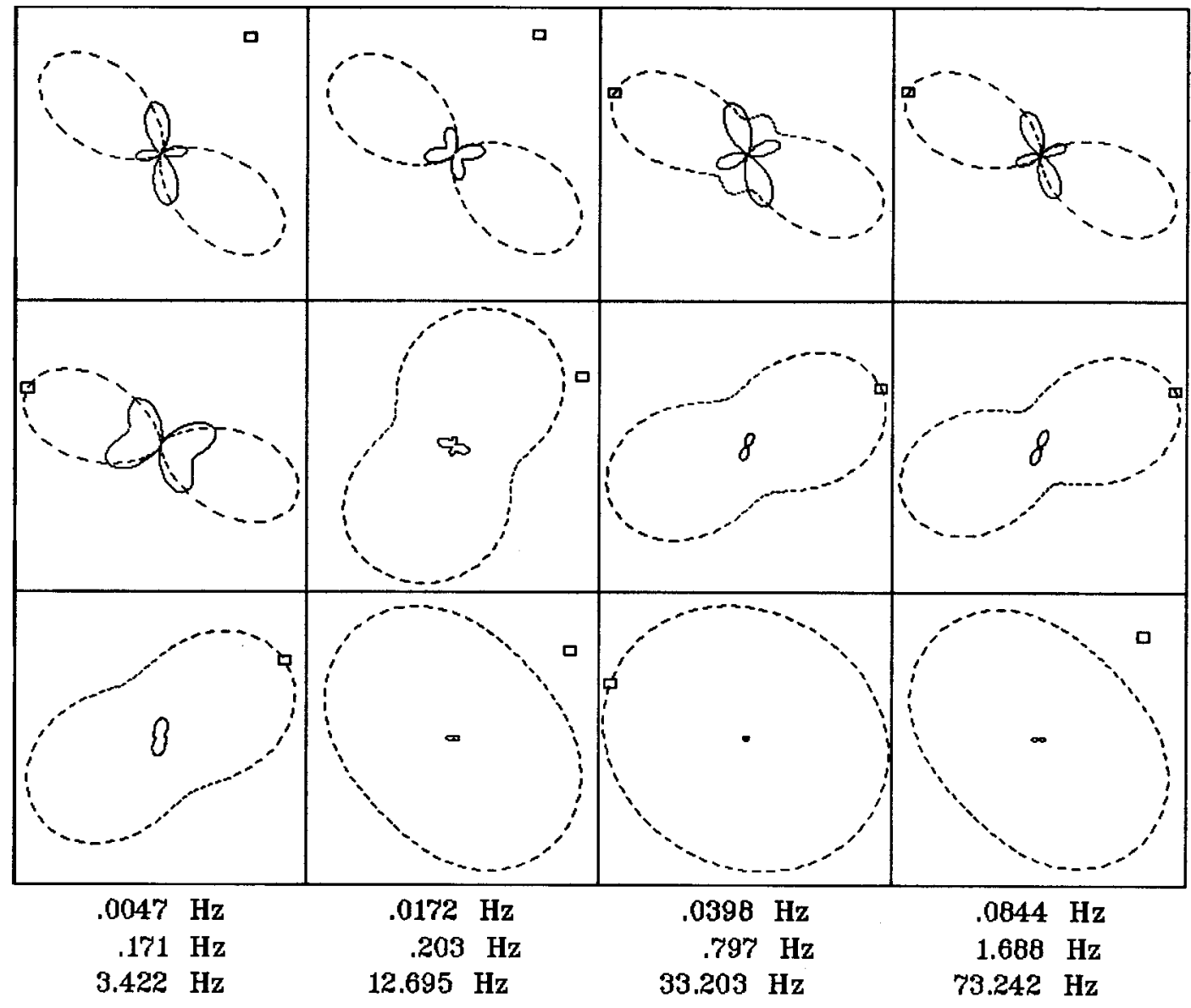

Client:

Remote:

Acquired:

Survey Co:
Rotation:

Filename: CT18

Channels: Ch1 Ch2 Ch3 Ch4 Ch5 Ch6 Ch7

Plotted: 10:14 Jun 25, 1999

$<$ EMI - ElectroMagnetic Instruments 
APPARENT RESISTIVITY Crescent Valley, NV 100K

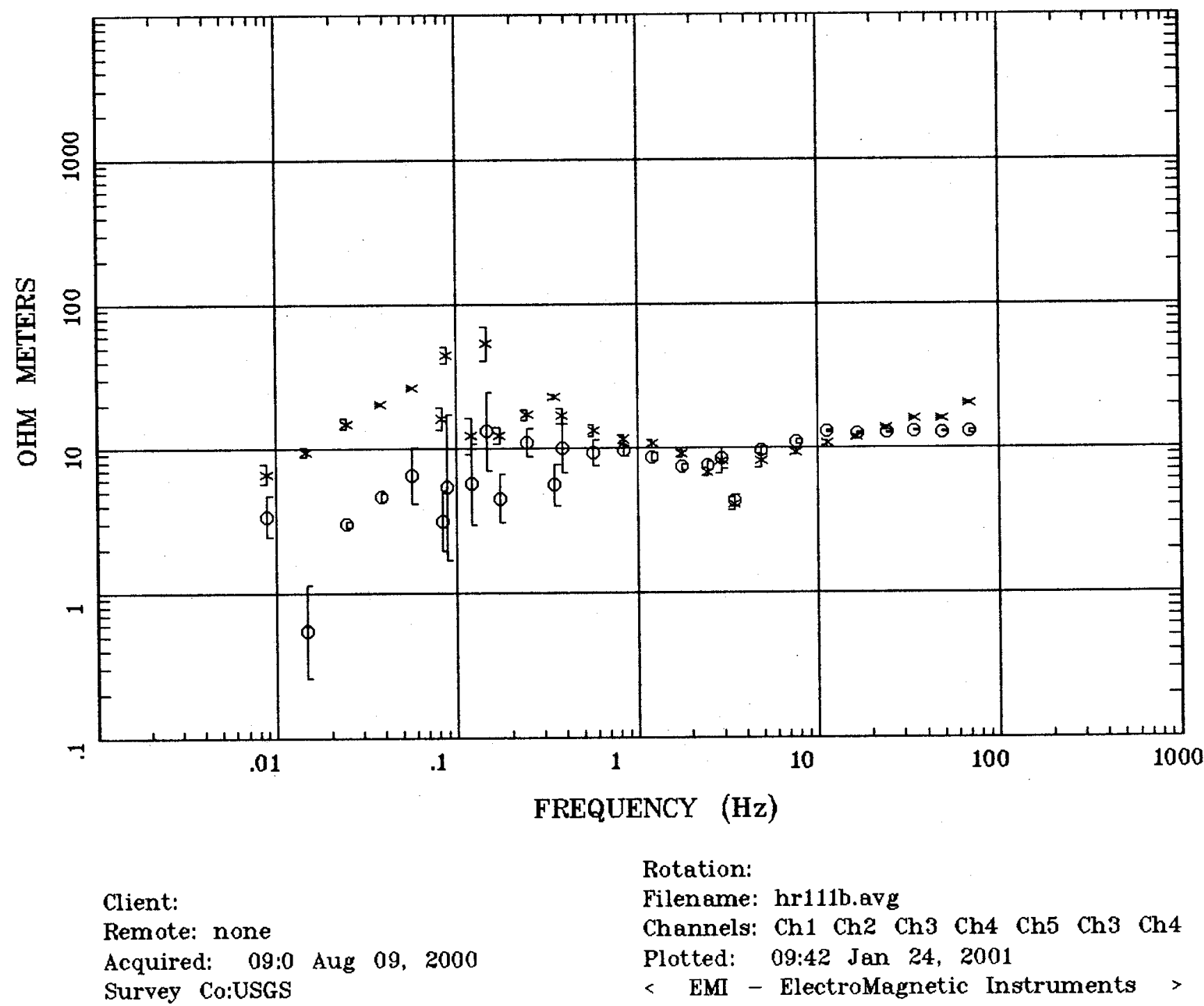


Station 111

IMPEDANCE PHASE

Crescent Valley, NV 100K

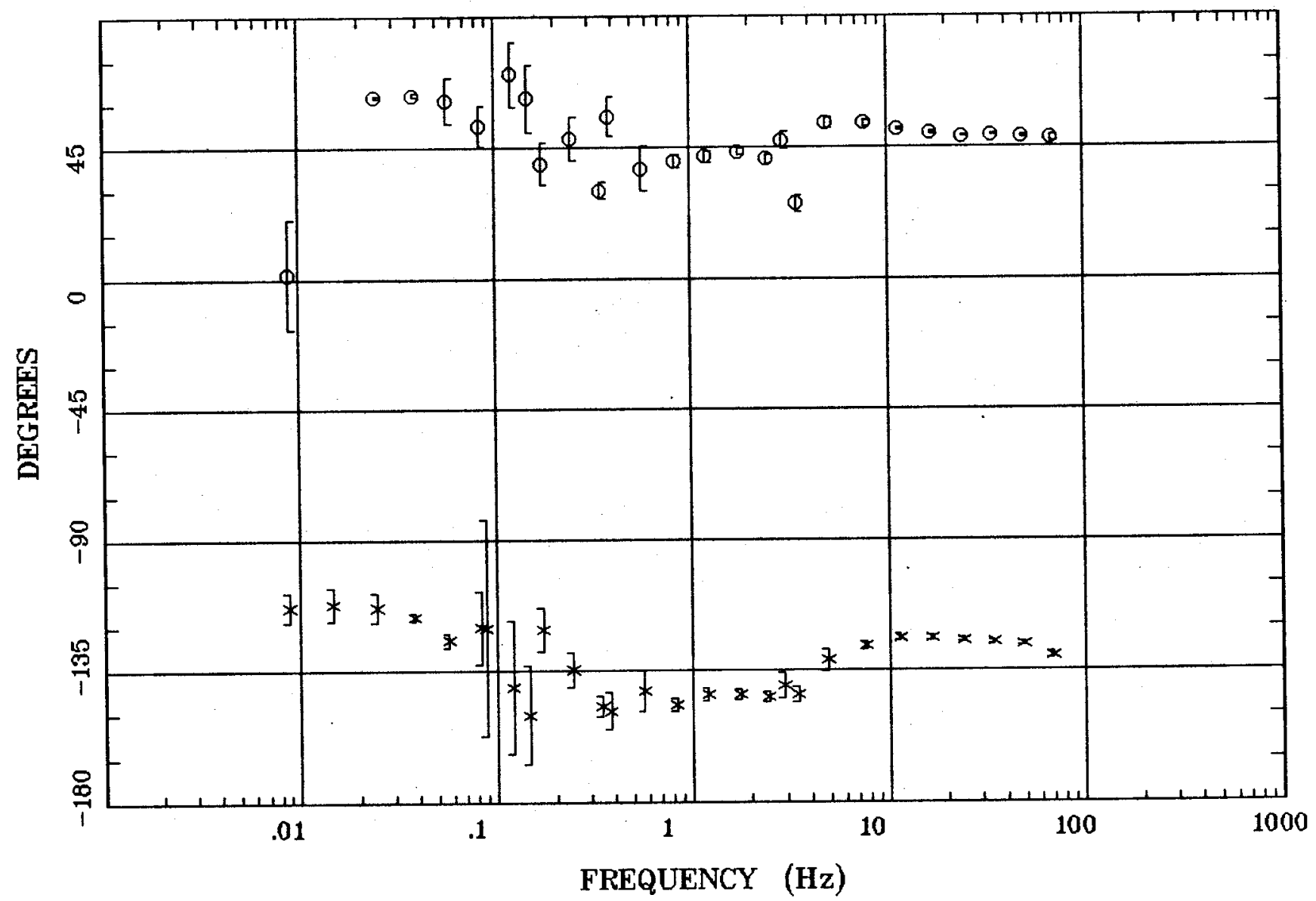

Client:

Remote: none

Acquired: 09:0 Aug 09, 2000 Survey co:USGS
Rotation:

Filename: hri11b.avg

Channels: Ch1 Ch2 Ch3 Ch4 Ch5 Ch3 Ch4

Plotted: 09:42 Jan 24, 2001

< EMI - ElectroMagnetic Instruments > 
Station 111

ROTATION ANGLE

Crescent Valley, NV 100K

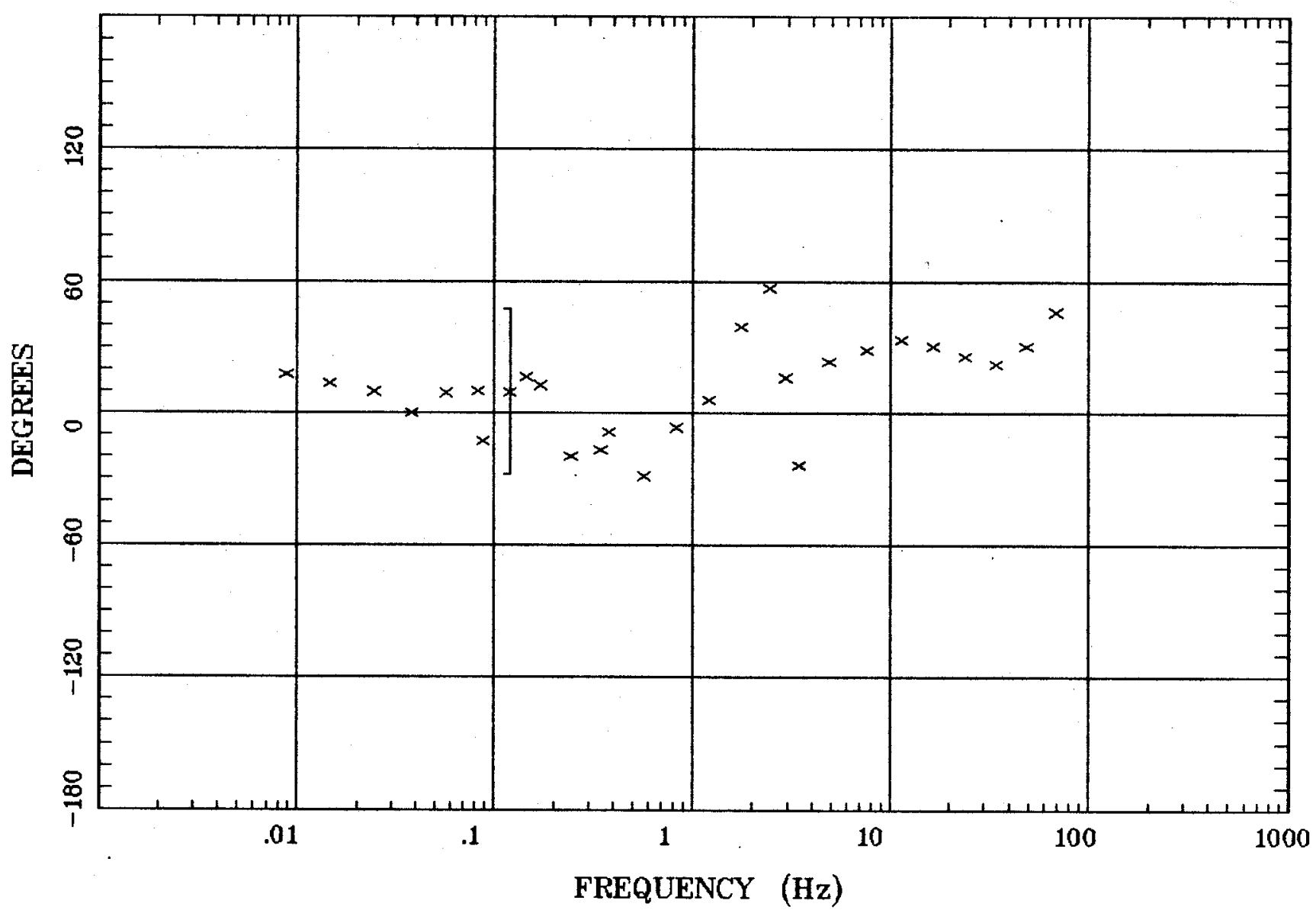

Client:

Remote: none

Acquired: 09:0 Aug 09, 2000 Survey Co:USGS
Rotation:

Filename: hrlib.avg

Channels: Ch1 Ch2 Ch3 Ch4 Ch5 Ch3 Ch4 Plotted: 09:42 Jan 24, 2001

$<$ EMI - ElectroMagnetic Instruments 
Station 111

IMPEDANCE SKEW

Crescent Valley, NV 100K

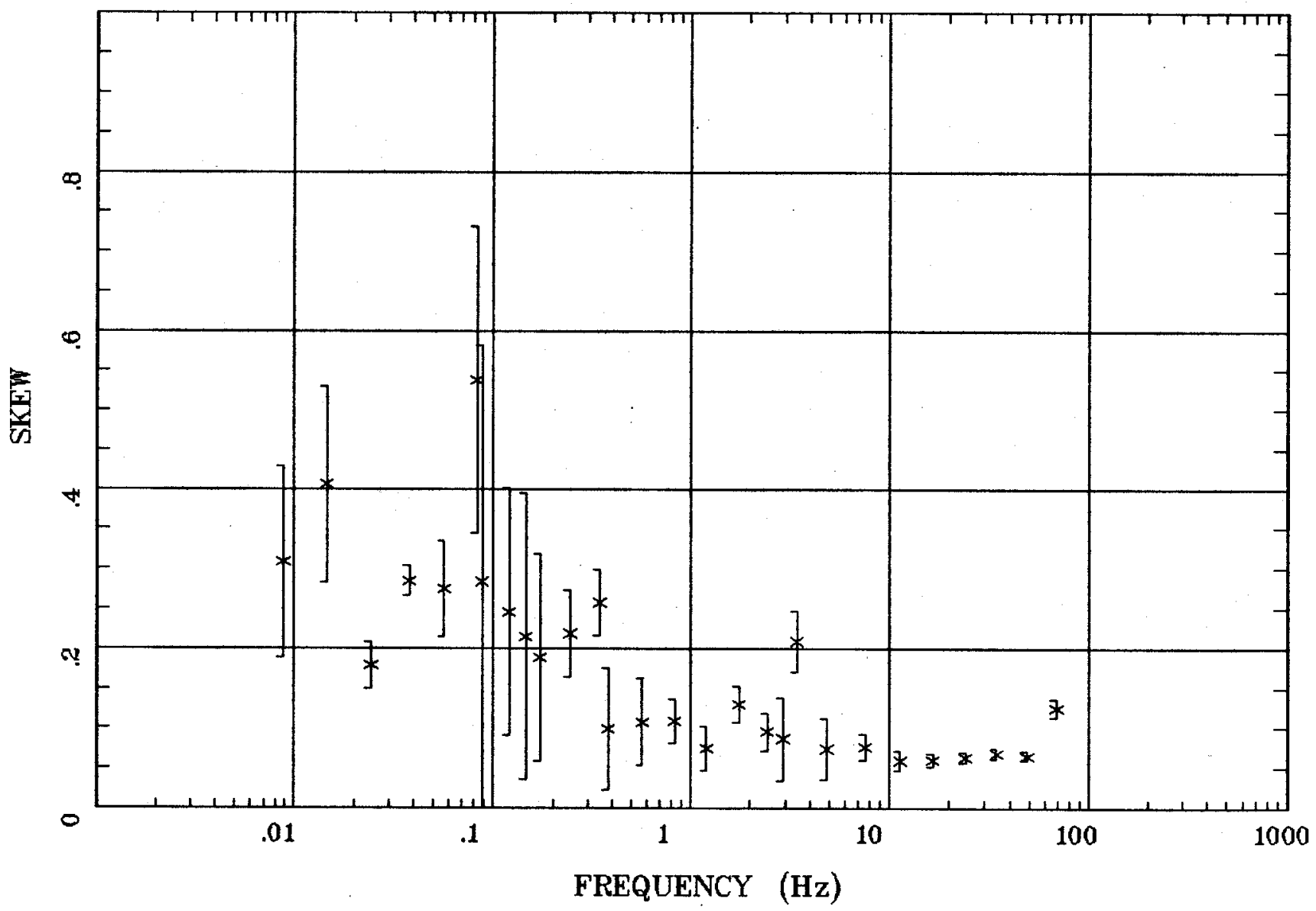

Client:

Remote: none

Acquired: 09:0 Aug 09, 2000 Survey Co:USGS
Rotation:

Filename: hr111b.avg

Channels: Ch1 Ch2 Ch3 Ch4 Ch5 Ch3 Ch4 Plotted: 09:42 Jan 24, 2001

< EMI - ElectroMagnetic Instruments 
E MULT Coh.

Crescent Valley, NV 100K

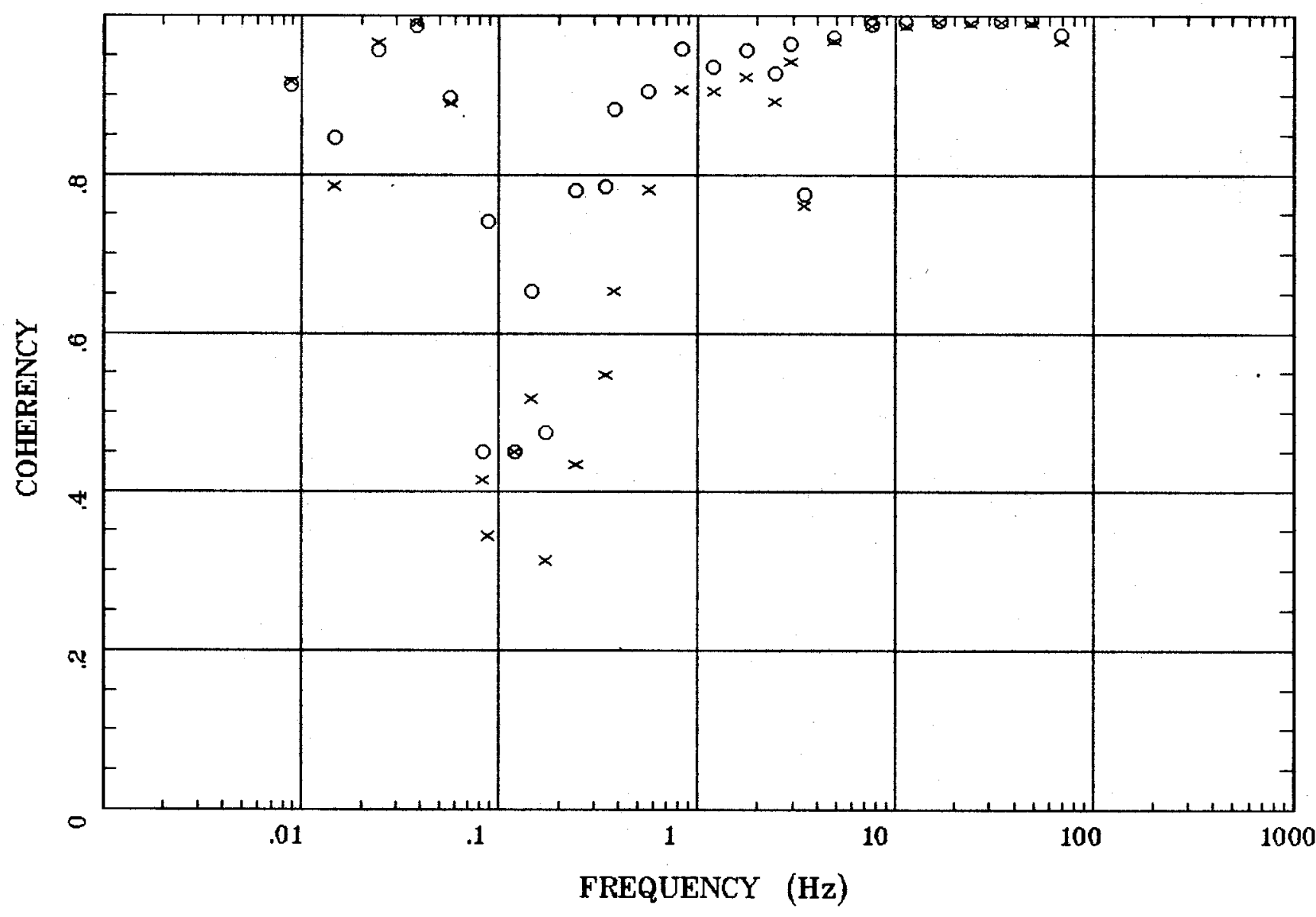

Client:

Remote: none

Acquired: 09:0 Aug 09, 2000

Survey Co:USGS
Rotation:

Filename: hr111b.avg

Channels: Ch1 Ch2 Ch3 Ch4 Ch5 Ch3 Ch4

Plotted: 09:42 Jan 24, 2001

$<$ EMI - ElectroMagnetic Instruments > 


\section{Station 111}

\section{POLAR PLOTS}

Crescent Valley, NV 100K

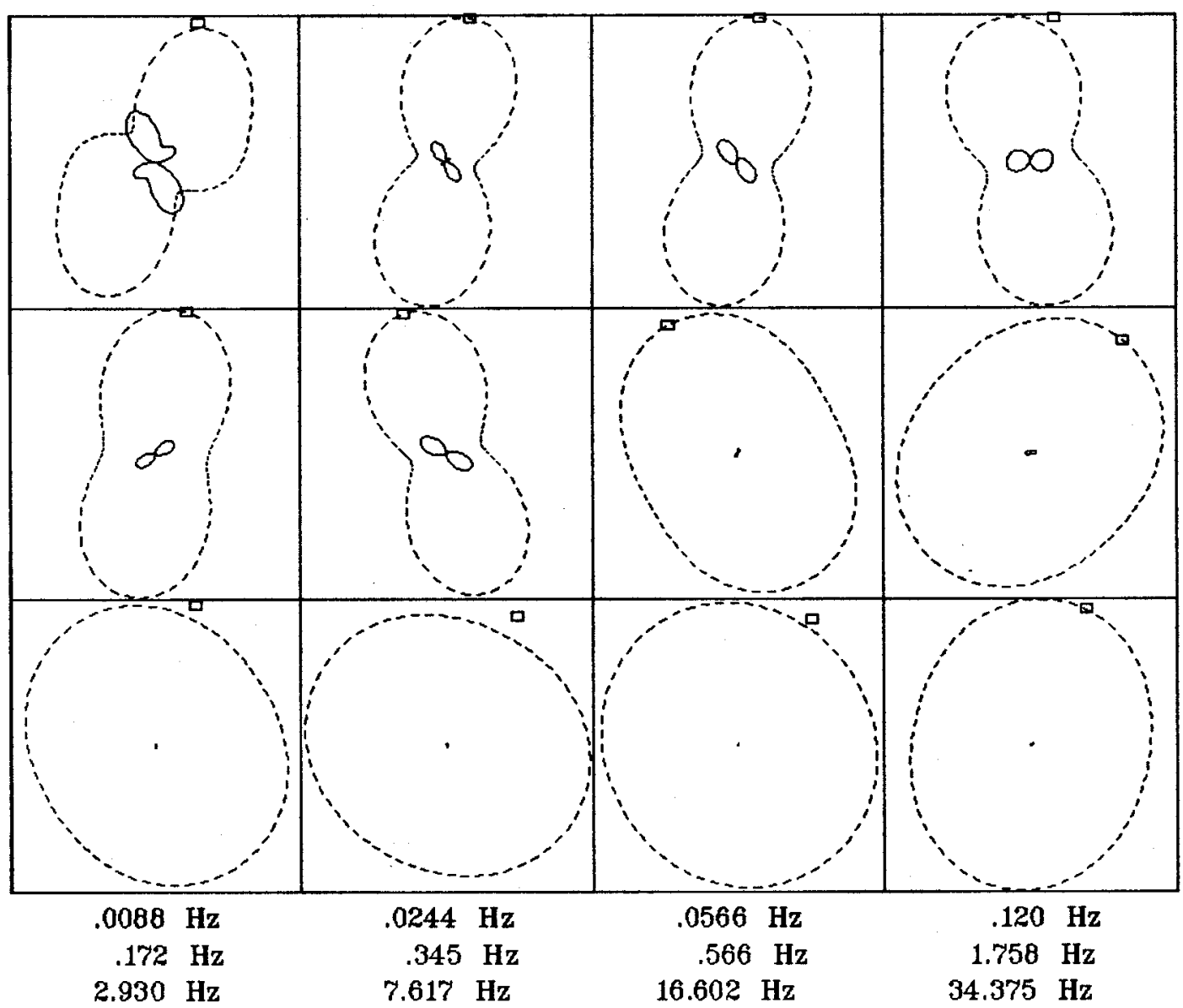

Client:

Rotation:

Remote: none

Acquired: 09:0 Aug 09, 2000

Filename: hr111b.avg

Survey Co:USGS

Channels: Ch1 Ch2 Ch3 Ch4 Ch5 Ch3 Ch4

Plotted: 09:42 Jan 24, 2001

< EMI - ElectroMagnetic Instruments > 


\section{Station 111}

TIPPER MAGNITUDE

Crescent Valley, NV 100K

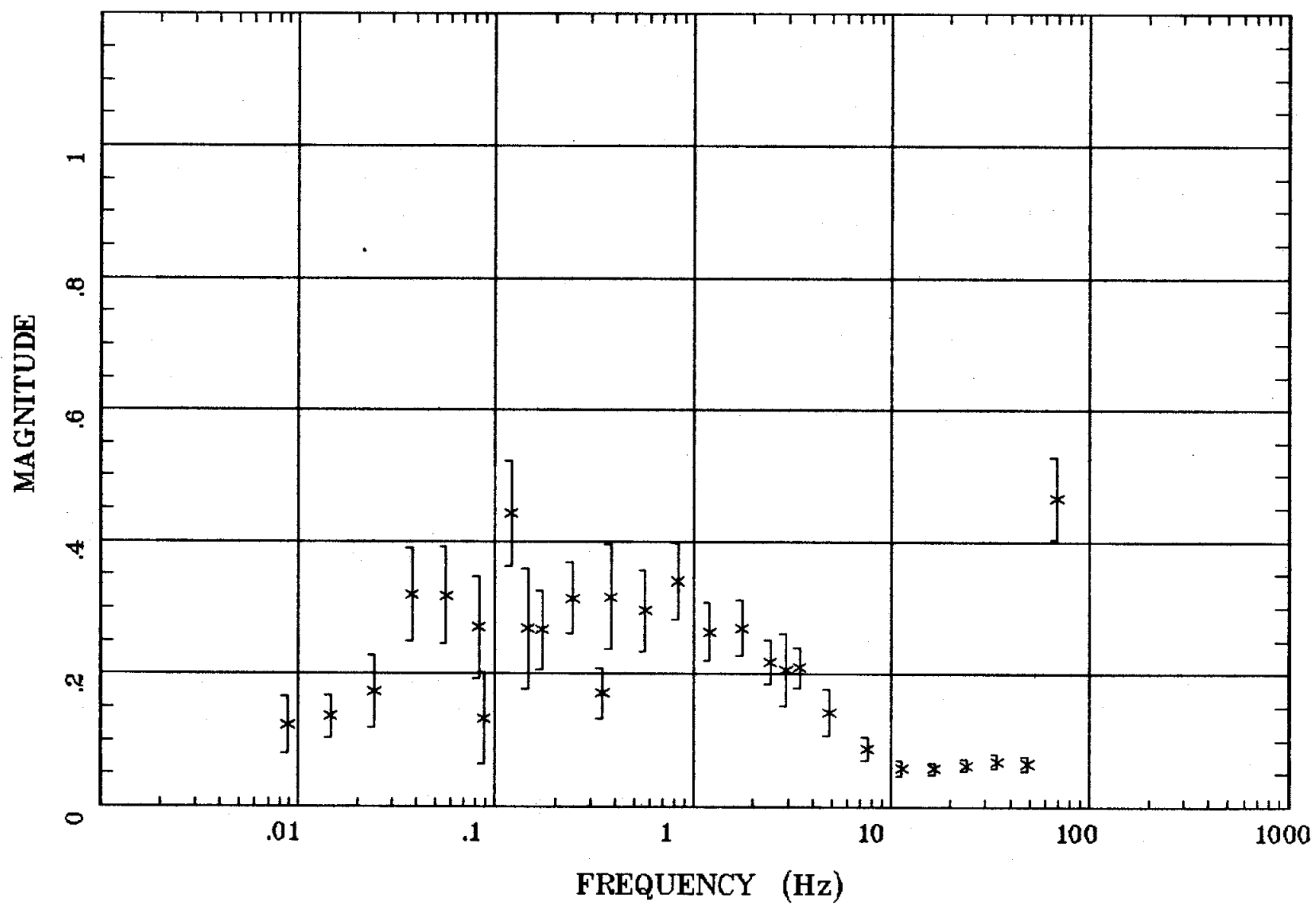

Client:

Remote: none

Acquired: 09:0 Aug 09, 2000 Survey Co:USGS
Rotation:

Filename: hr111b.avg

Channels: Ch1 Ch2 Ch3 Ch4 Ch5 Ch3 Ch4 Plotted: 09:42 Jan 24, 2001

$<$ EMI - ElectroMagnetic Instruments 
TIPPER STRIKE

Crescent Valley, NV 100K

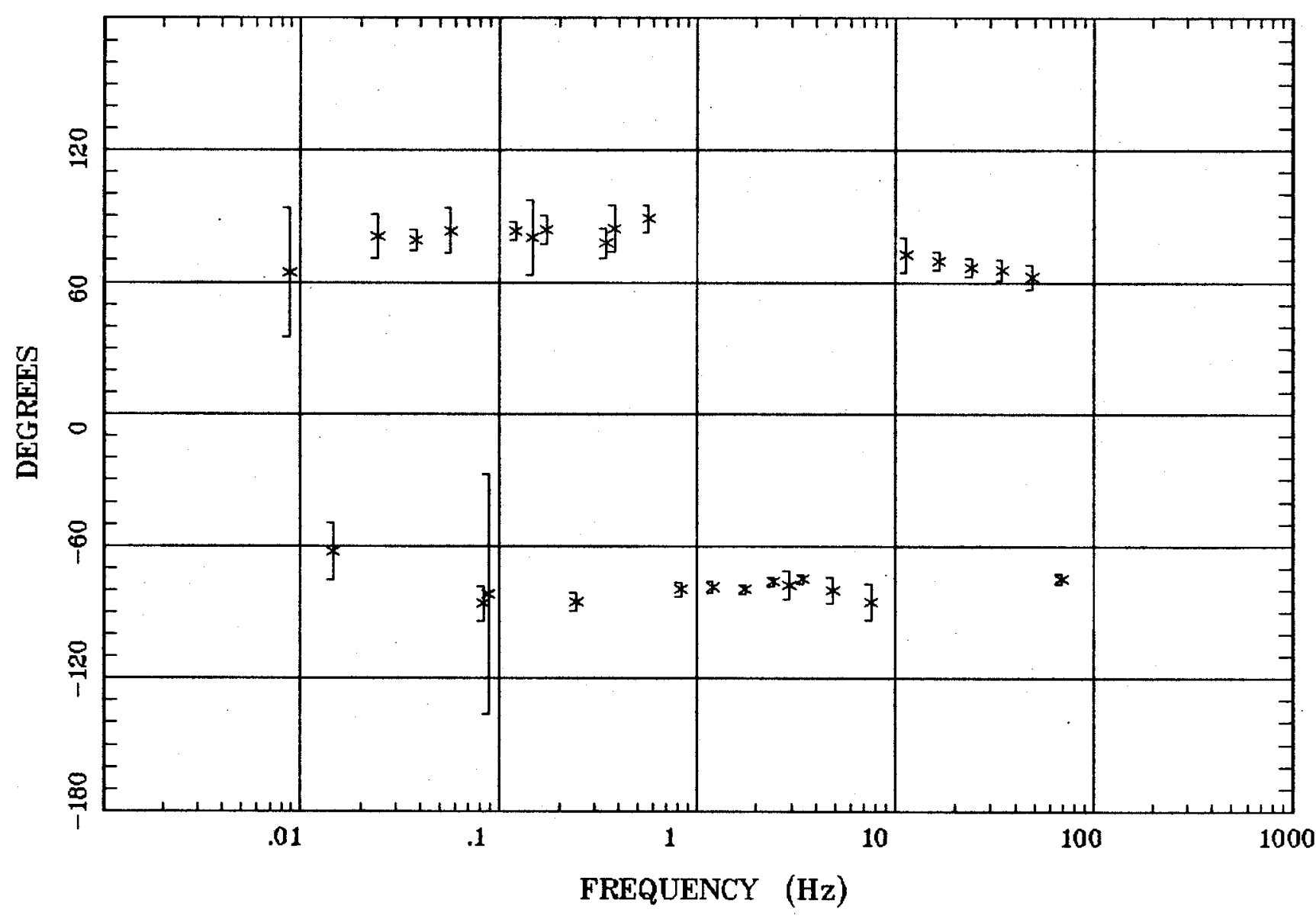

Client:

Remote: none

Acquired: 09:0 Aug 09, 2000 Survey Co:USGS
Rotation:

Filename: hr111b.avg

Channels: Ch1 Ch2 Ch3 Ch4 Ch5 Ch3 Ch4

Plotted: 09:42 Jan 24, 2001

$<$ EMI - ElectroMagnetic Instruments 
HzHx.x Coh HzHy.o

Crescent Valley, NV $100 \mathrm{~K}$

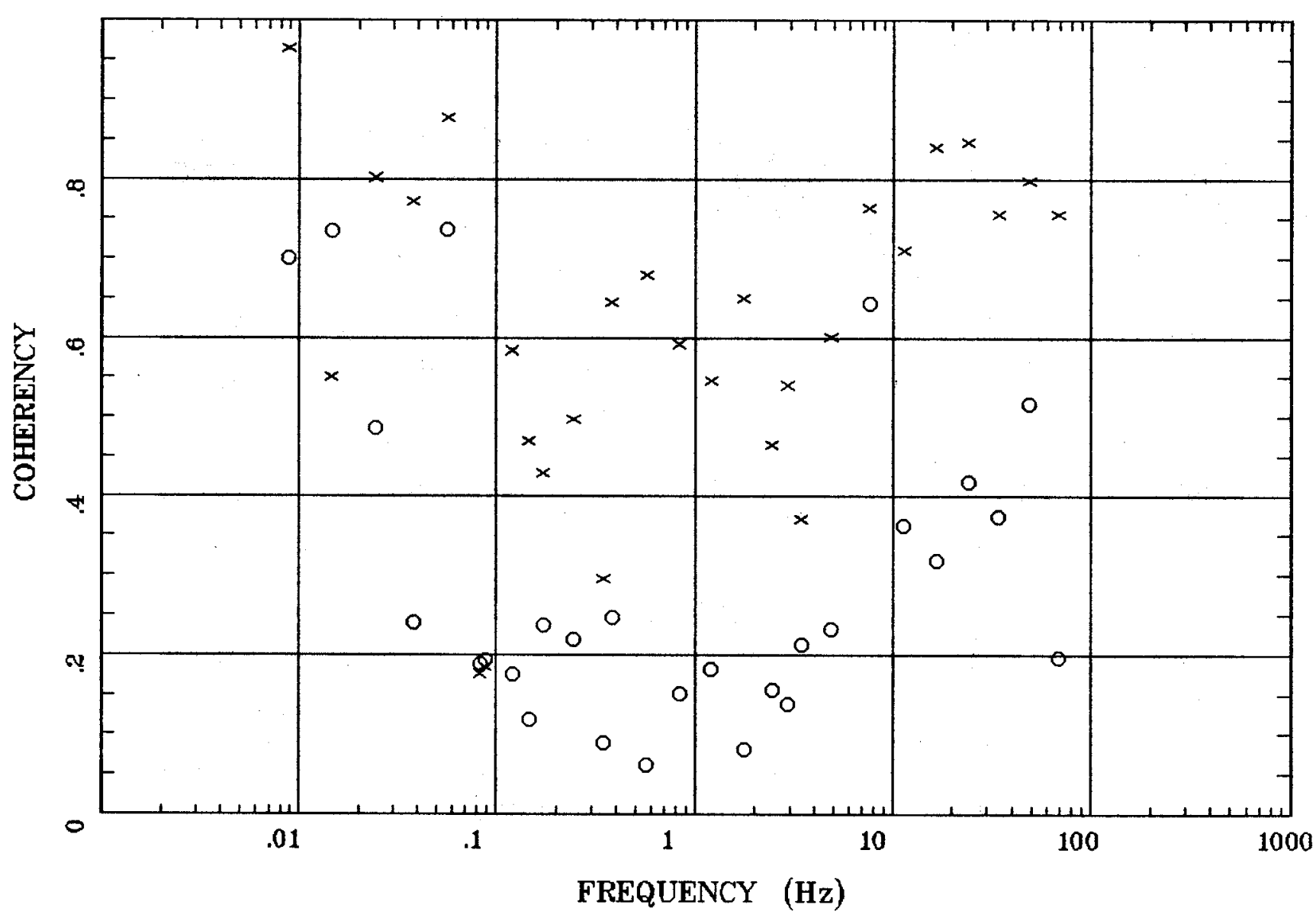

Client:

Remote: none

Acquired: 09:0 Aug 09, 2000

Survey Co:USGS
Rotation:

Filename: hr111b.avg

Channels: Ch1 Ch2 Ch3 Ch4 Ch5 Ch3 Ch4

Plotted: 09:42 Jan 24, 2001

$<$ EMI - ElectroMagnetic Instruments 
APPARENT RESISTIVITY

Ruby Lake NV 100k

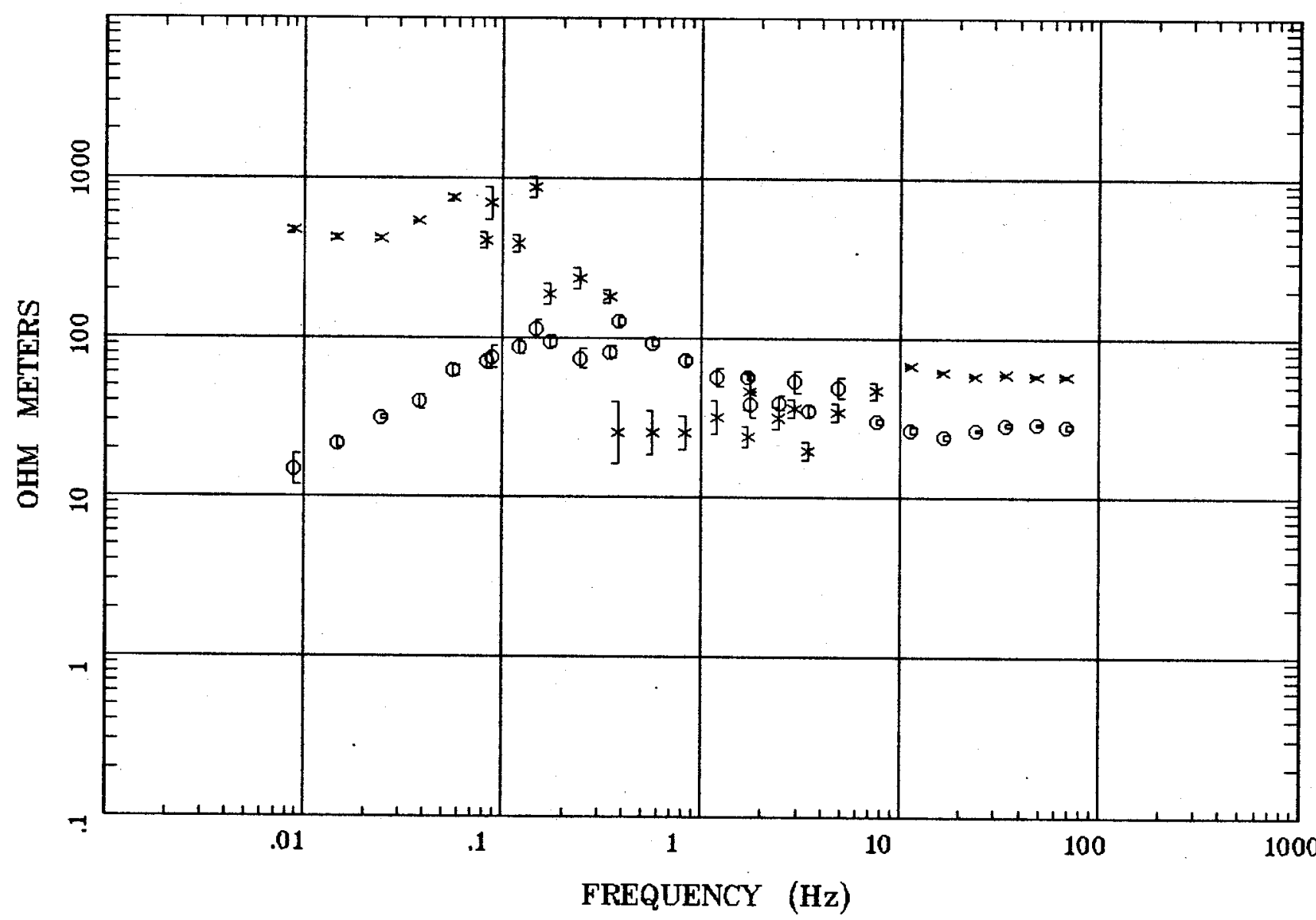

Client:

Remote: none

Acquired: 13:3 Aug 08, 2000

Survey Co:USGS
Rotation:

Filename: hr110all.avg

Channels: Ch1 Ch2 Ch3 Ch4 Ch5 Ch3 Ch4

Plotted: 09:43 Jan 24, 2001

$<$ EMI - ElectroMagnetic Instruments 


\section{Station 110}

IMPEDANCE PHASE

Ruby Lake NV 100k

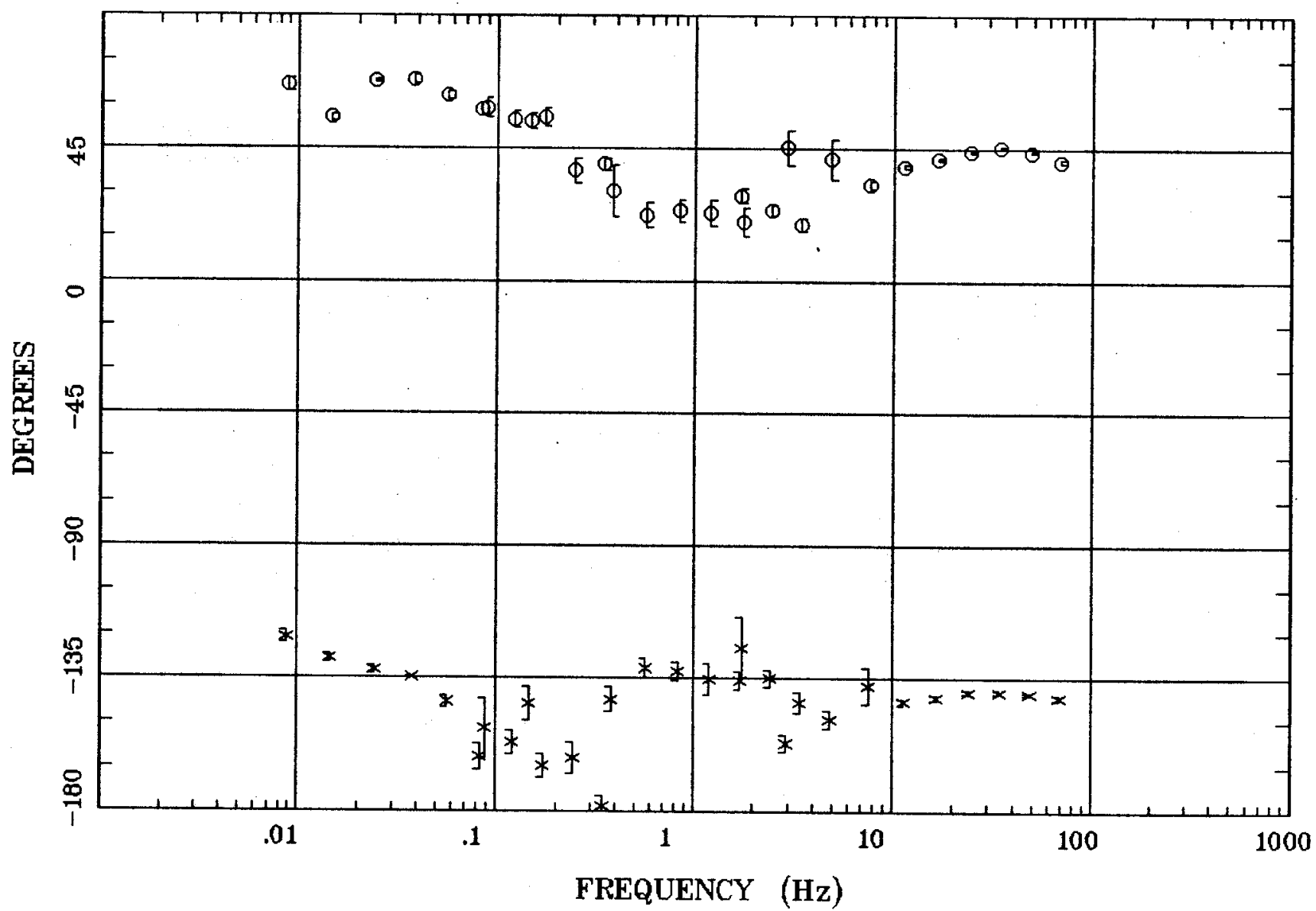

Client:

Remote: none

Acquired: 13:3 Aug 08, 2000

Survey Co:USGS

\section{Rotation:}

Filename: hr110all.avg

Channels: Ch1 Ch2 Ch3 Ch4 Ch5 Ch3 Ch4

Plotted: 09:43 Jan 24, 2001

< EMI - ElectroMagnetic Instruments > 
Station 110

ROTATION ANGLE

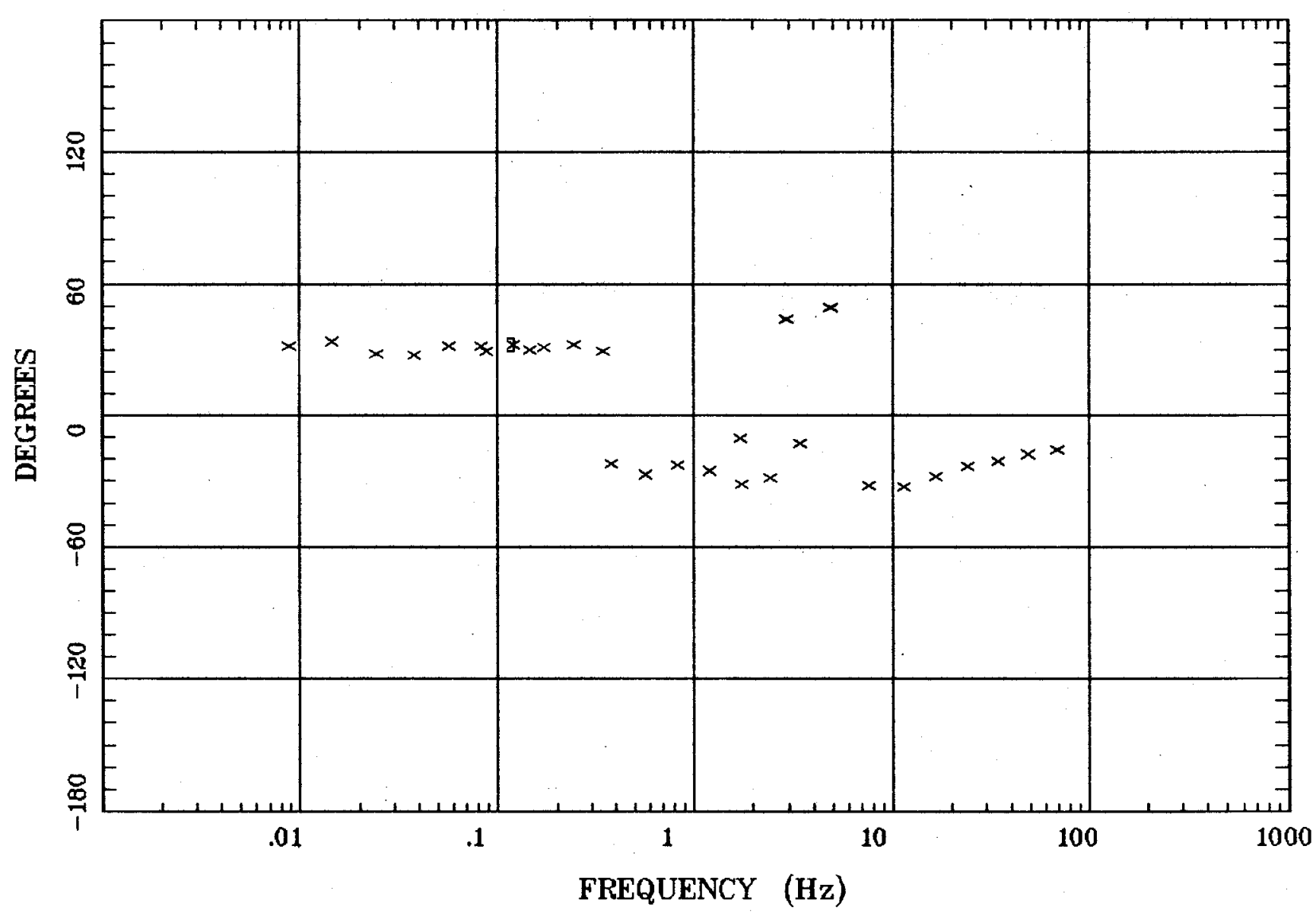

Ruby Lake NV 100k

\section{Client:}

Remote: none

Acquired: 13:3 Aug 08, 2000

Survey Co:USGS
Rotation:

Filename: hr110all.avg

Channels: Ch1 Ch2 Ch3 Ch4 Ch5 Ch3 Ch4

Plotted: 09:43 Jan 24, 2001

< EMI - ElectroMagnetic Instruments > 
Ruby Lake NV 100k

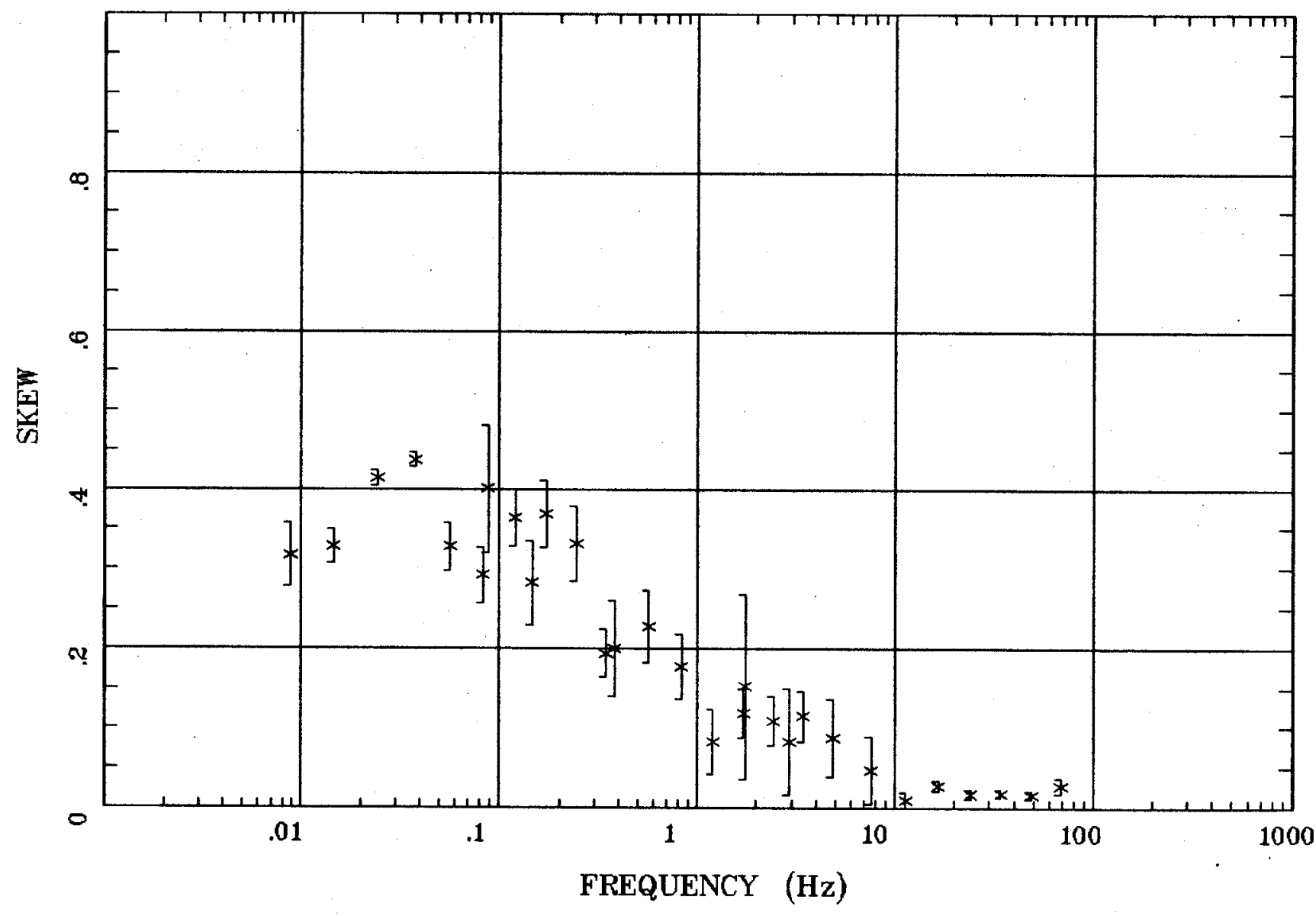

Client:

Remote: none

Acquired: 13:3 Aug 08, 2000 Survey Co:USGS
Rotation:

Filename: hr110all.avg

Channels: Ch1 Ch2 Ch3 Ch4 Ch5 Ch3 Ch4

Plotted: 09:43 Jan 24, 2001

$<$ EMI - ElectroMagnetic Instruments 
E MULT Coh.

Ruby Lake NV 100k

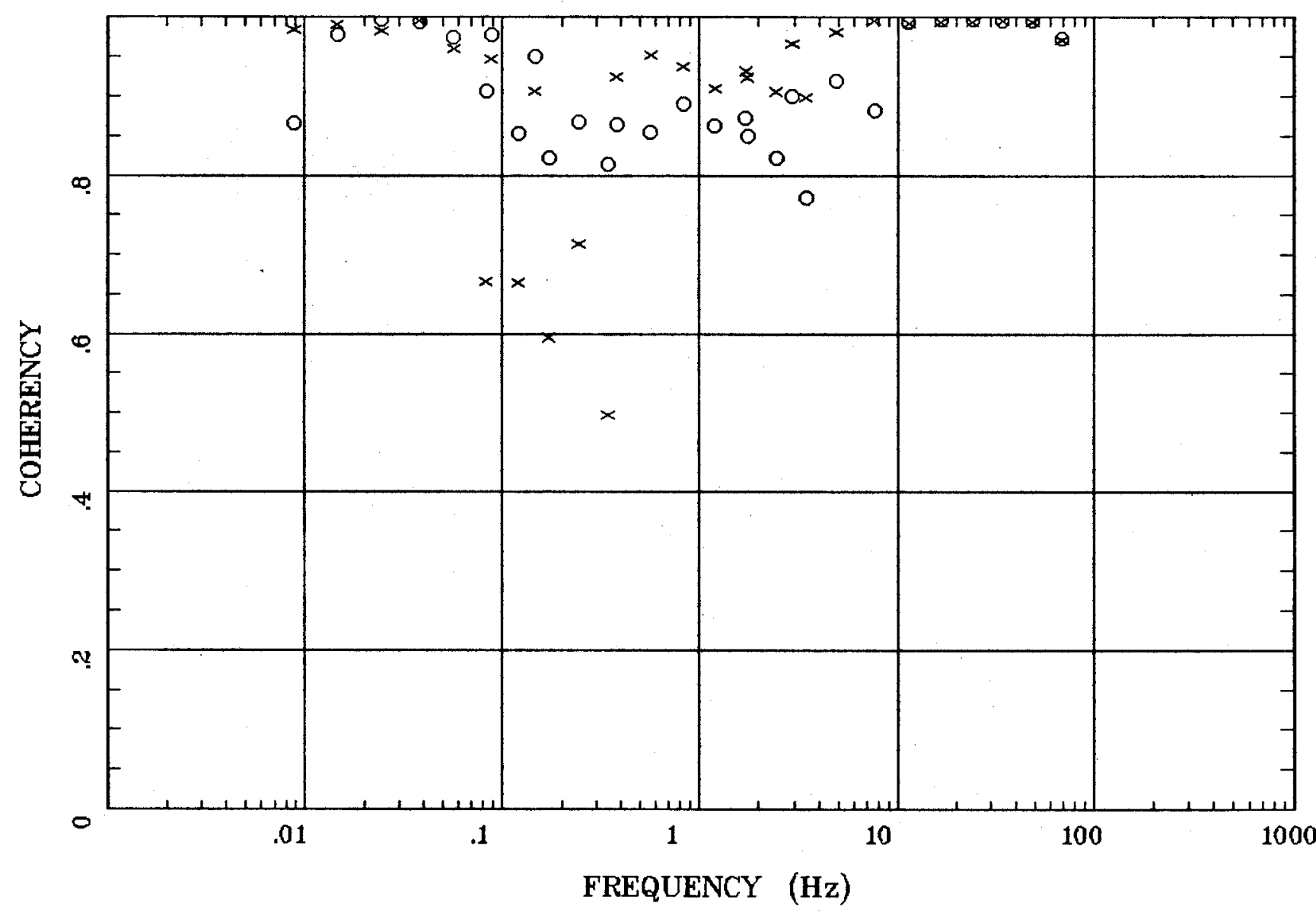

Client:

Remote: none

Acquired: 13:3 Aug 08, 2000

Survey Co:USGS
Rotation:

Filename: hr110all.avg

Channels: Ch1 Ch2 Ch3 Ch4 Ch5 Ch3 Ch4

Plotted: $\quad 09: 43$ Jan 24, 2001

< EMI - ElectroMagnetic Instruments > 
POLAR PLOTS

Ruby Lake NV 100k

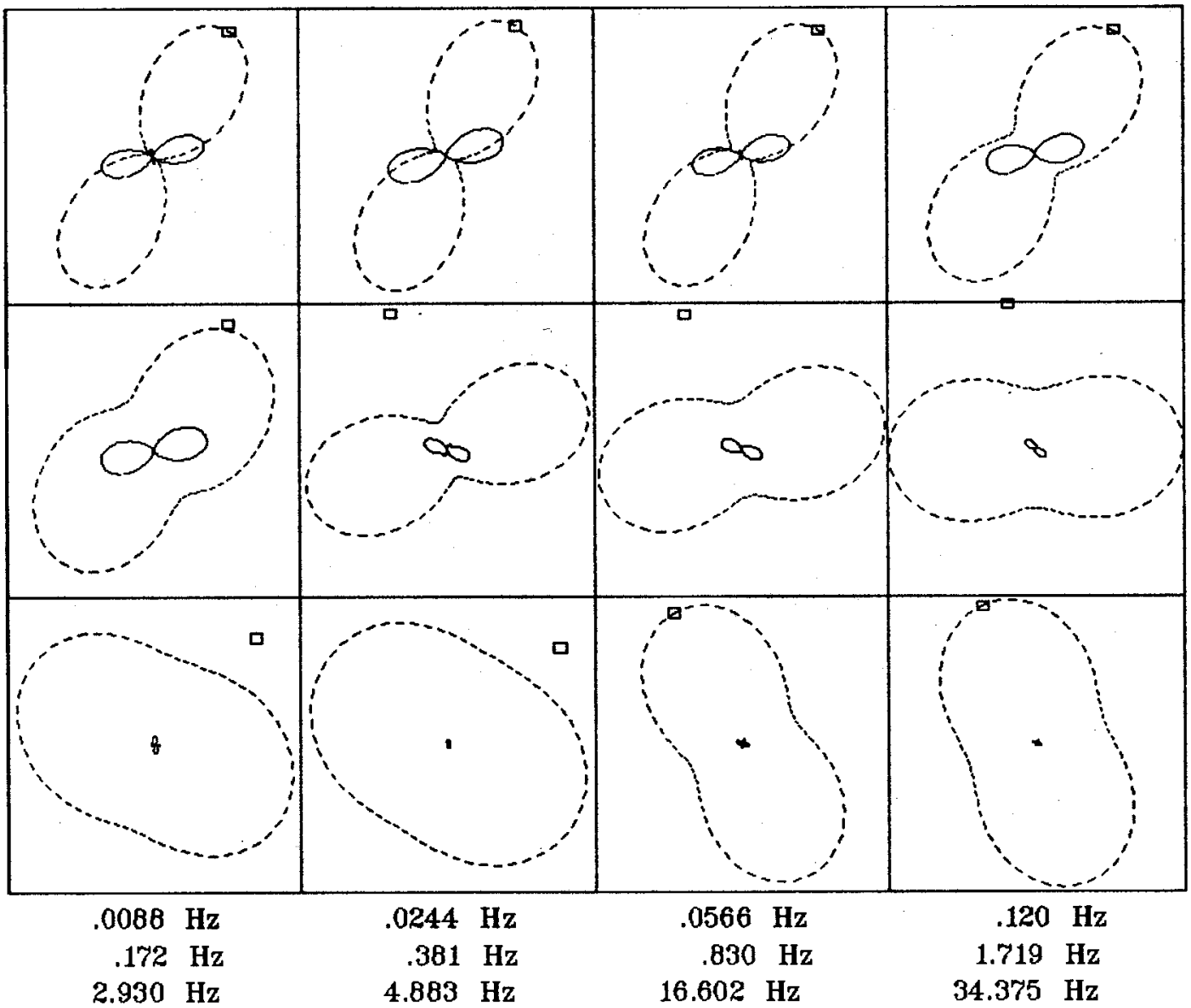

Client:

Remote: none

Acquired: 13:3 Aug 08, 2000 Survey Co:USGS
Rotation:

Filename: hr110all.avg

Channels: Ch1 Ch2 Ch3 Ch4 Ch5 Ch3 Ch4

Plotted: 09:43 Jan 24, 2001

$<$ EMI - ElectroMagnetic Instruments 
TIPPER MAGNITUDE

Ruby Lake NV 100k

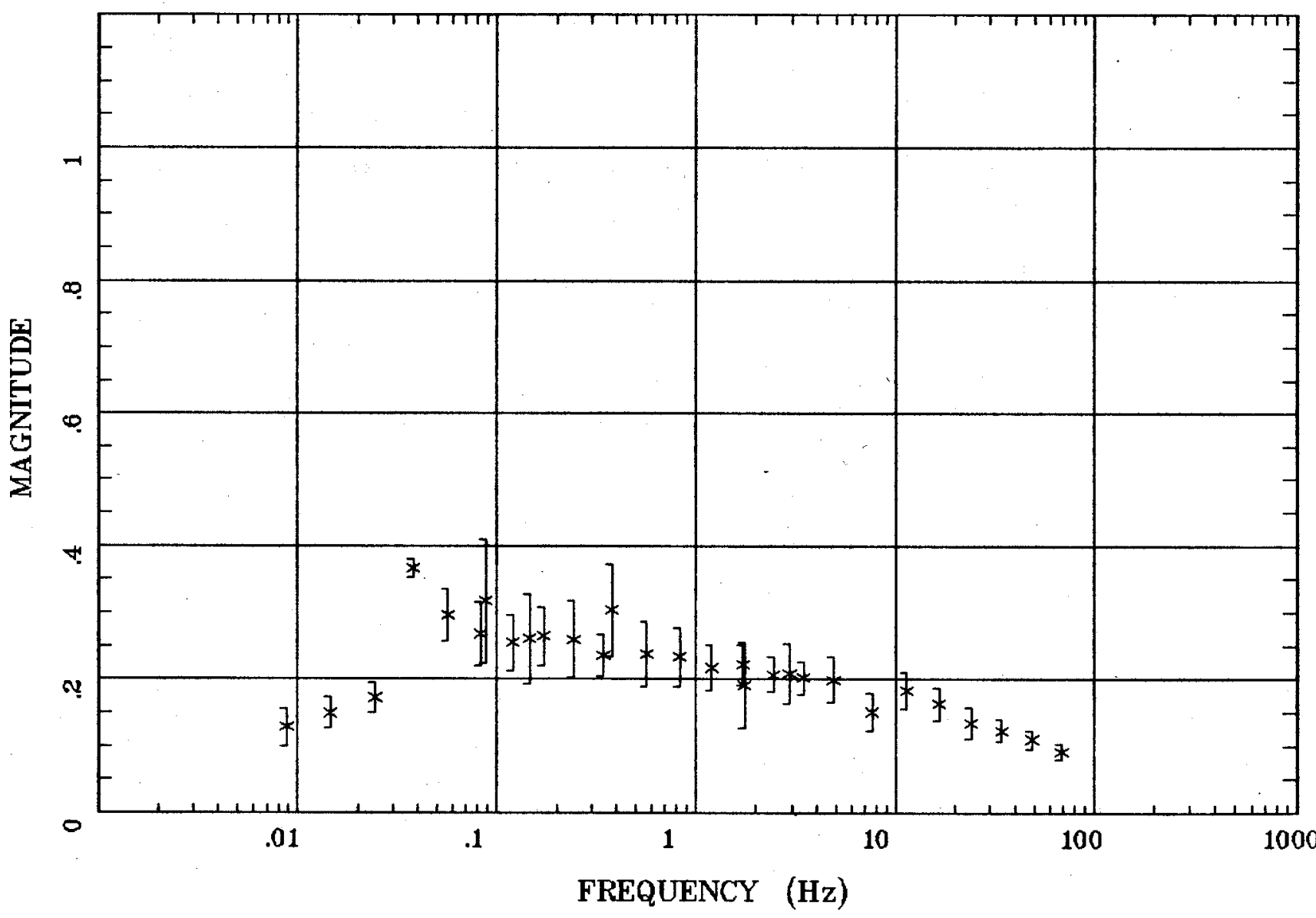

Client:

Remote: none

Acquired: 13:3 Aug 08, 2000 Survey Co:USGS
Rotation:

Filename: hr110all.avg

Channels: Ch1 Ch2 Ch3 Ch4 Ch5 Ch3 Ch4

Plotted: 09:43 Jan 24, 2001

< EMI - ElectroMagnetic Instruments 
TIPPER STRIKE

Ruby Lake NV 100k

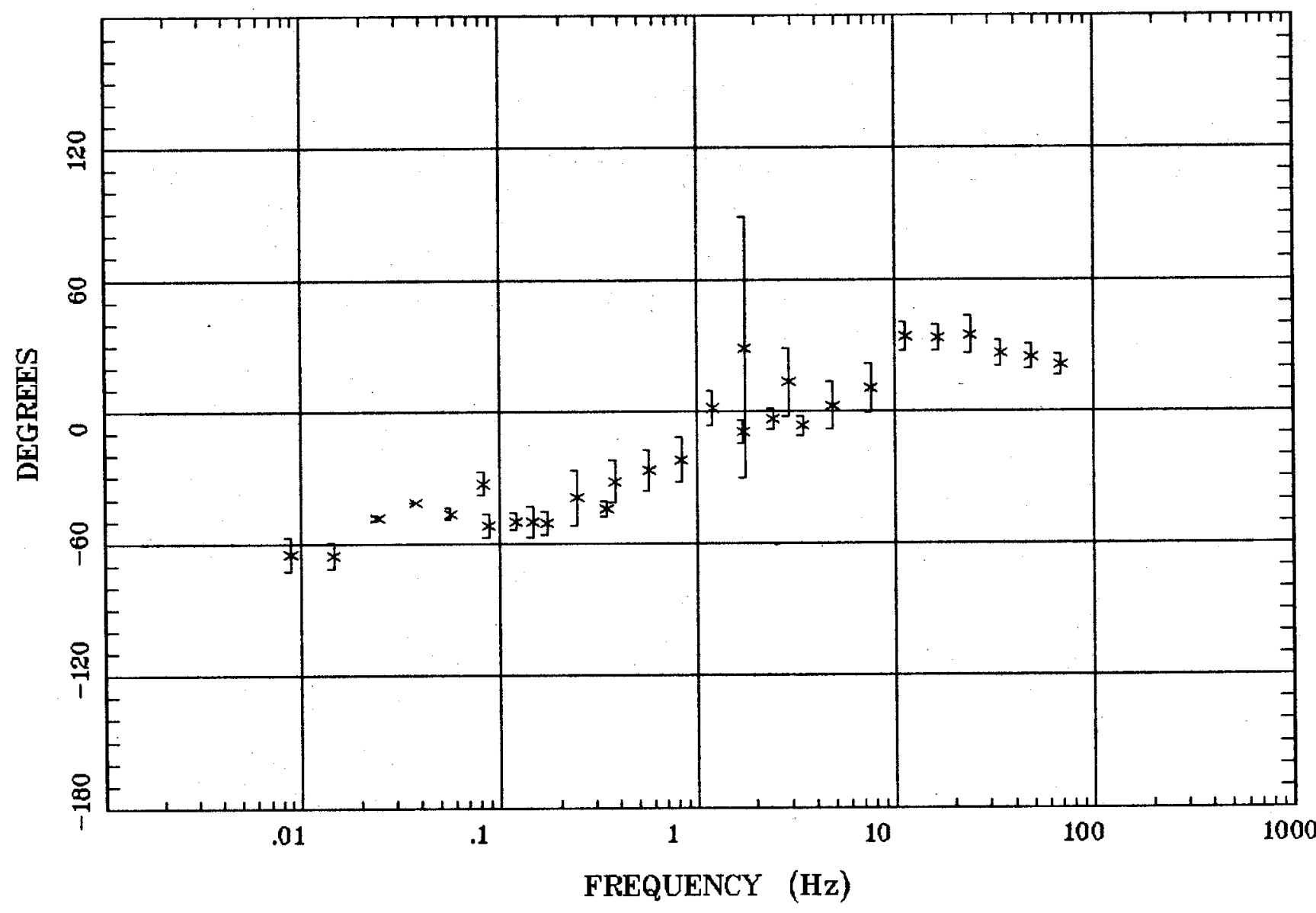

Client:

Remote: none

Acquired: 13:3 Aug 08, 2000 Survey Co:USGS
Rotation:

Filename: hr110all.avg

Channels: Ch1 Ch2 Ch3 ch4 Ch5 Ch3 Ch4 Plotted: 09:43 Jan 24, 2001

- EMI - ElectroMagnetic Instruments 
HzHx.x Coh HzHy.O

Ruby Lake NV 100k

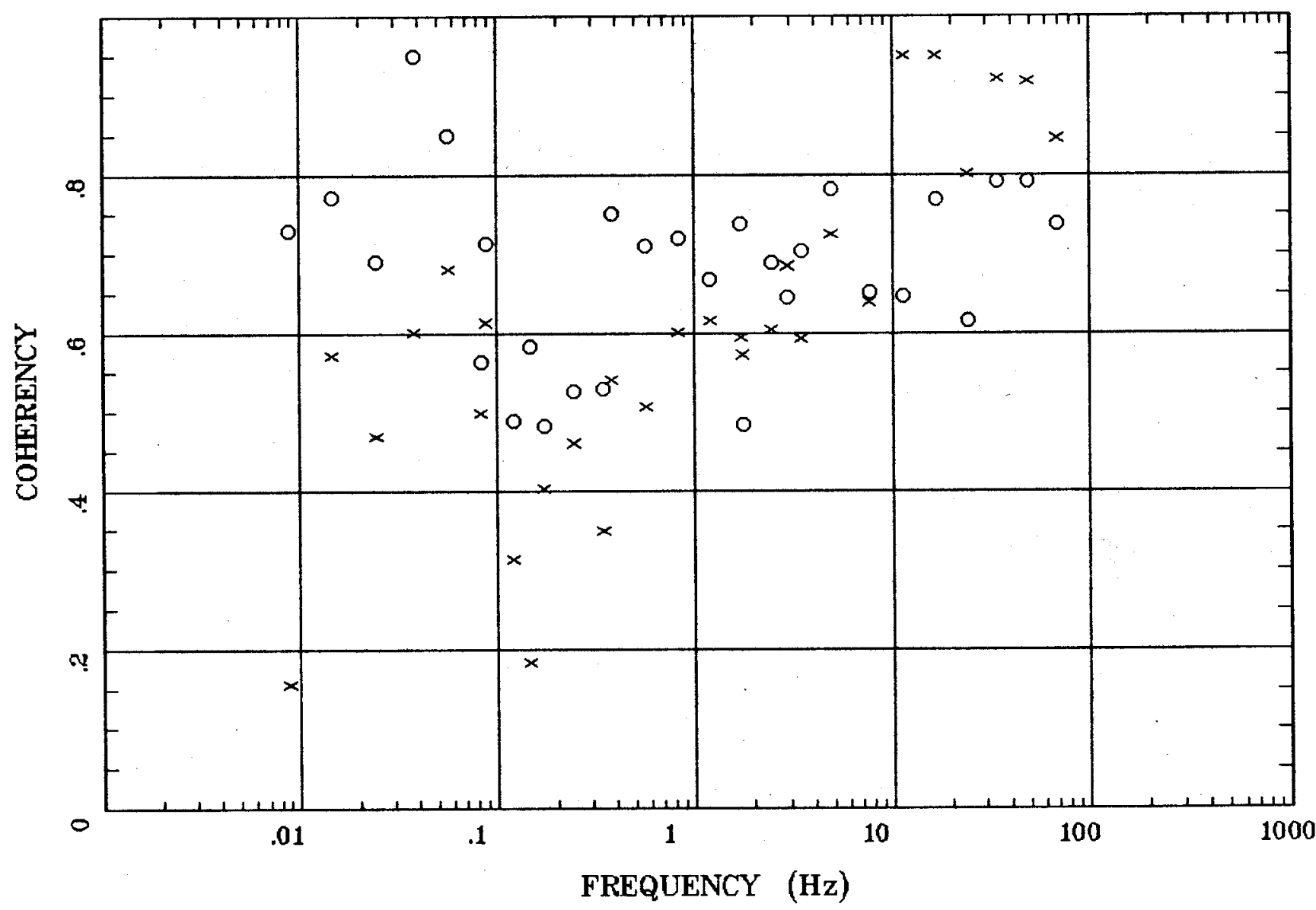

Client:

Remote: none

Acquired: 13:3 Aug 08, 2000

Survey Co:USGS
Rotation:

Filename: hr110all.avg

Channels: Ch1 Ch2 Ch3 Ch4 Ch5 Ch3 Ch4

Plotted: 09:43 Jan 24, 2001

$<$ EMI - ElectroMagnetic Instruments 
Station 112

\section{APPARENT RESISTIVITY Ruby Lake NV 100k}

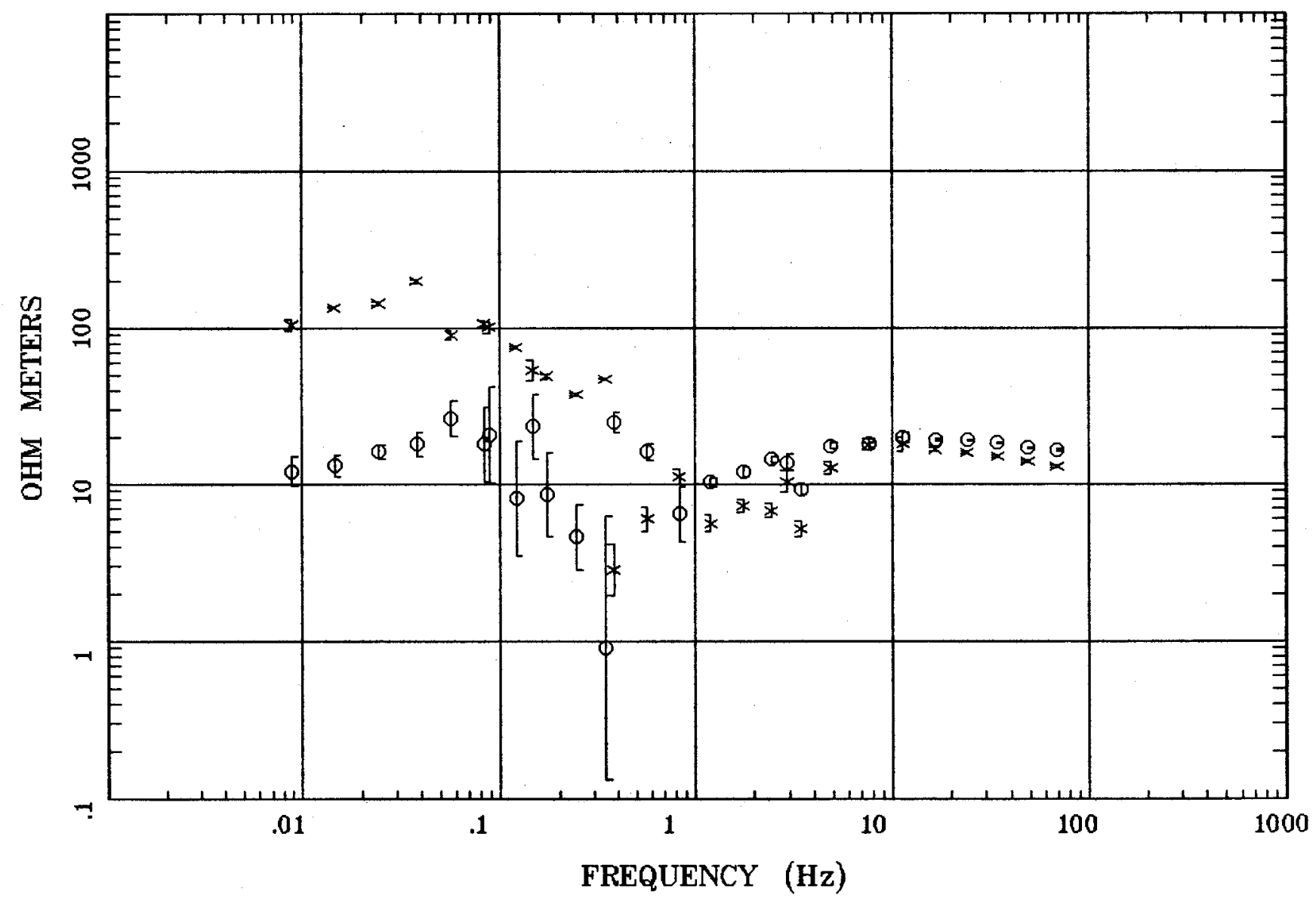

Client:

Remote: none

Acquired: 13:3 Aug 09, 2000 Survey Co:USGS

\section{Rotation:}

Filename: hr112b.avg

Channels: Ch1 Ch2 Ch3 Ch4 Ch5 Ch3 Ch4 Plotted: 09:44 Jan 24, 2001

< EMI - ElectroMagnetic Instruments > 
IMPEDANCE PHASE

Ruby Lake NV 100k

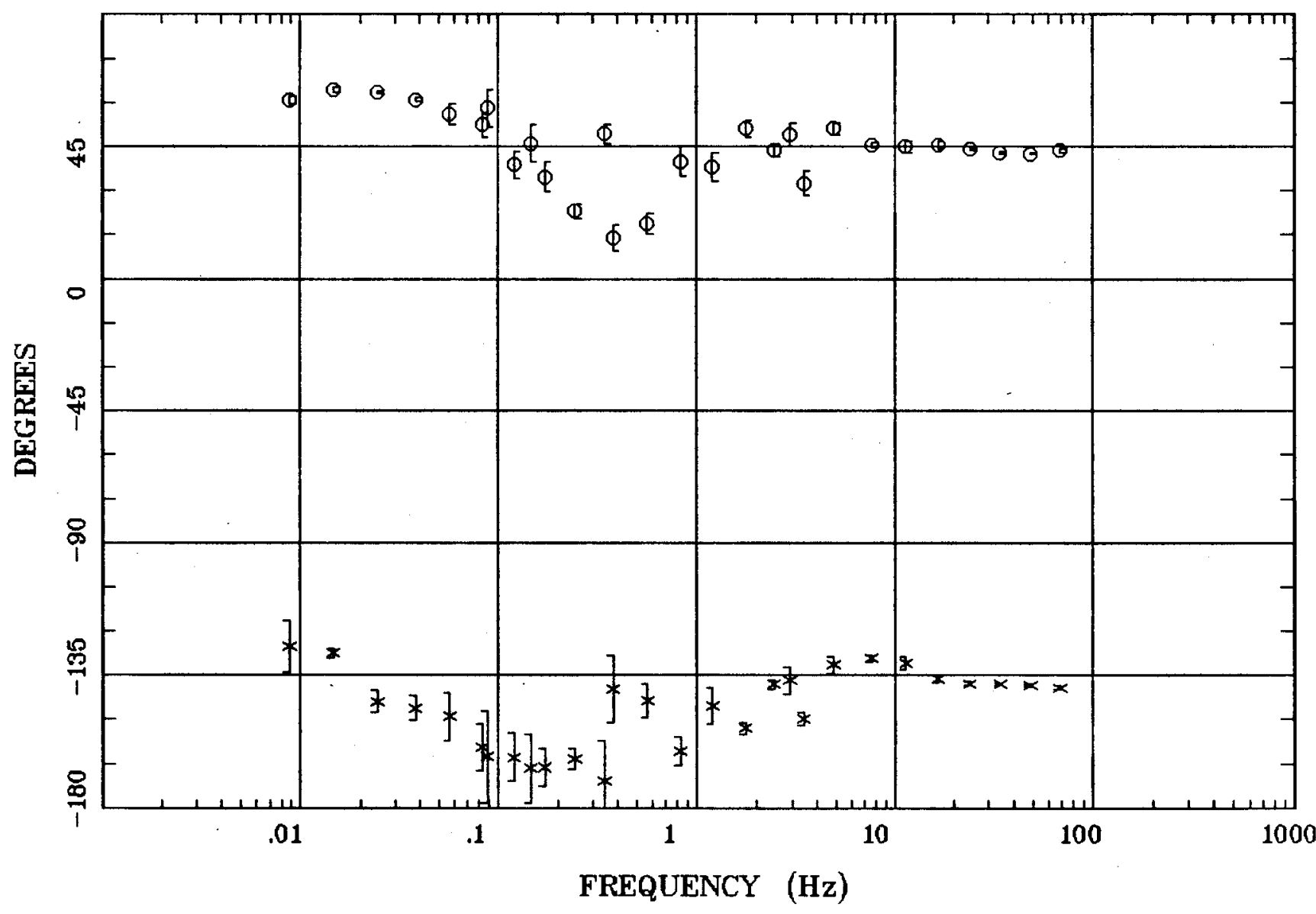

Client:

Remote: none

Acquired: 13:3 Aug 09, 2000

Survey Co:USGS

Rotation:

Filename: hr112b.avg

Channels: Ch1 Ch2 Ch3 Ch4 Ch5 Ch3 Ch4

Plotted: 09:44 Jan 24, 2001

$<$ EMI - ElectroMagnetic Instruments 
ROTATION ANGLE

Ruby Lake NV 100k

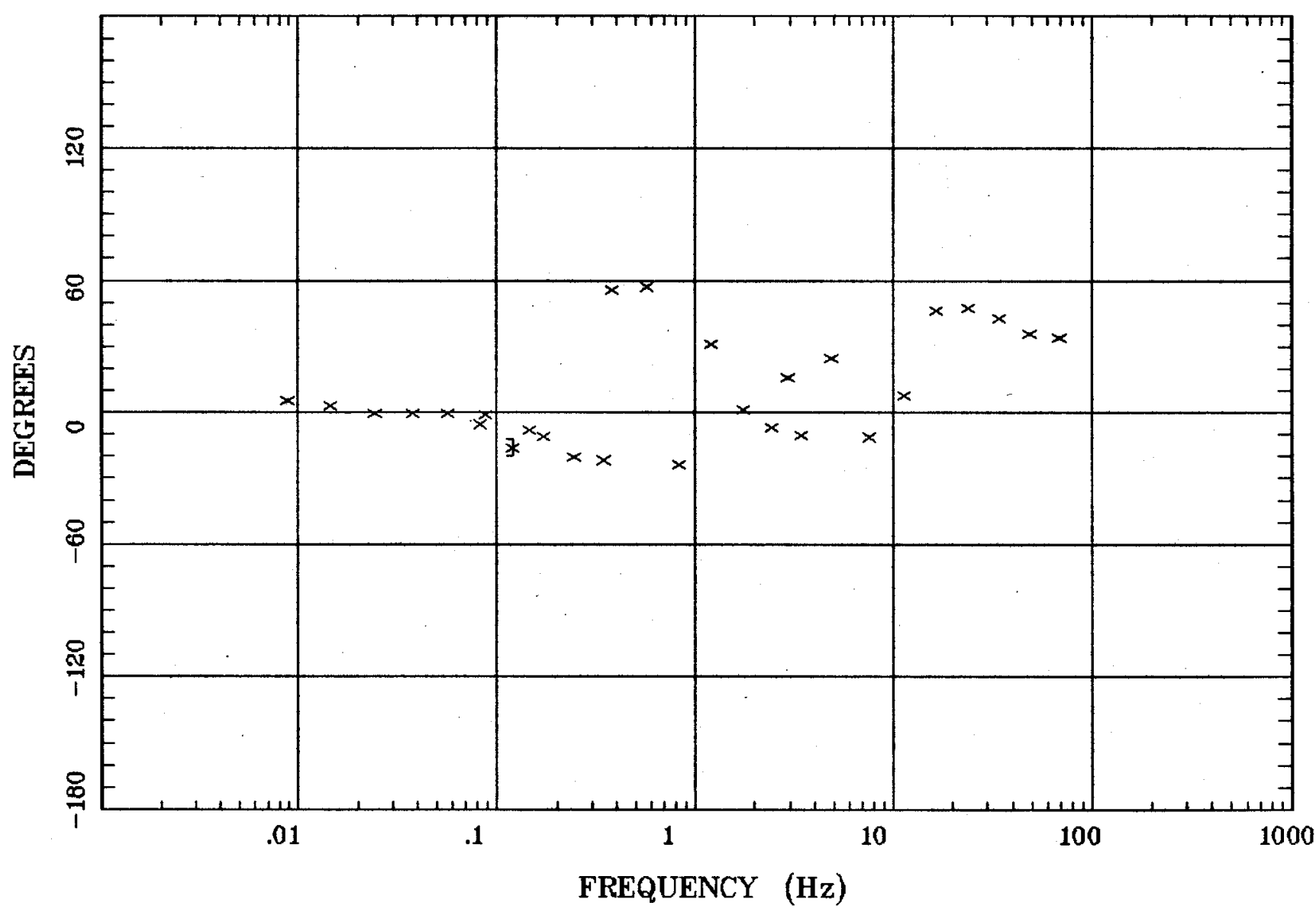

Client:

Remote: none

Acquired: 13:3 Aug 09, 2000 Survey Co:USGS
Rotation:

Filename: hr112b.avg

Channels: Ch1 Ch2 Ch3 Ch4 Ch5 Ch3 Ch4 Plotted: 09:44 Jan 24, 2001

< EMI - ElectroMagnetic Instruments 
Ruby Lake NV 100k

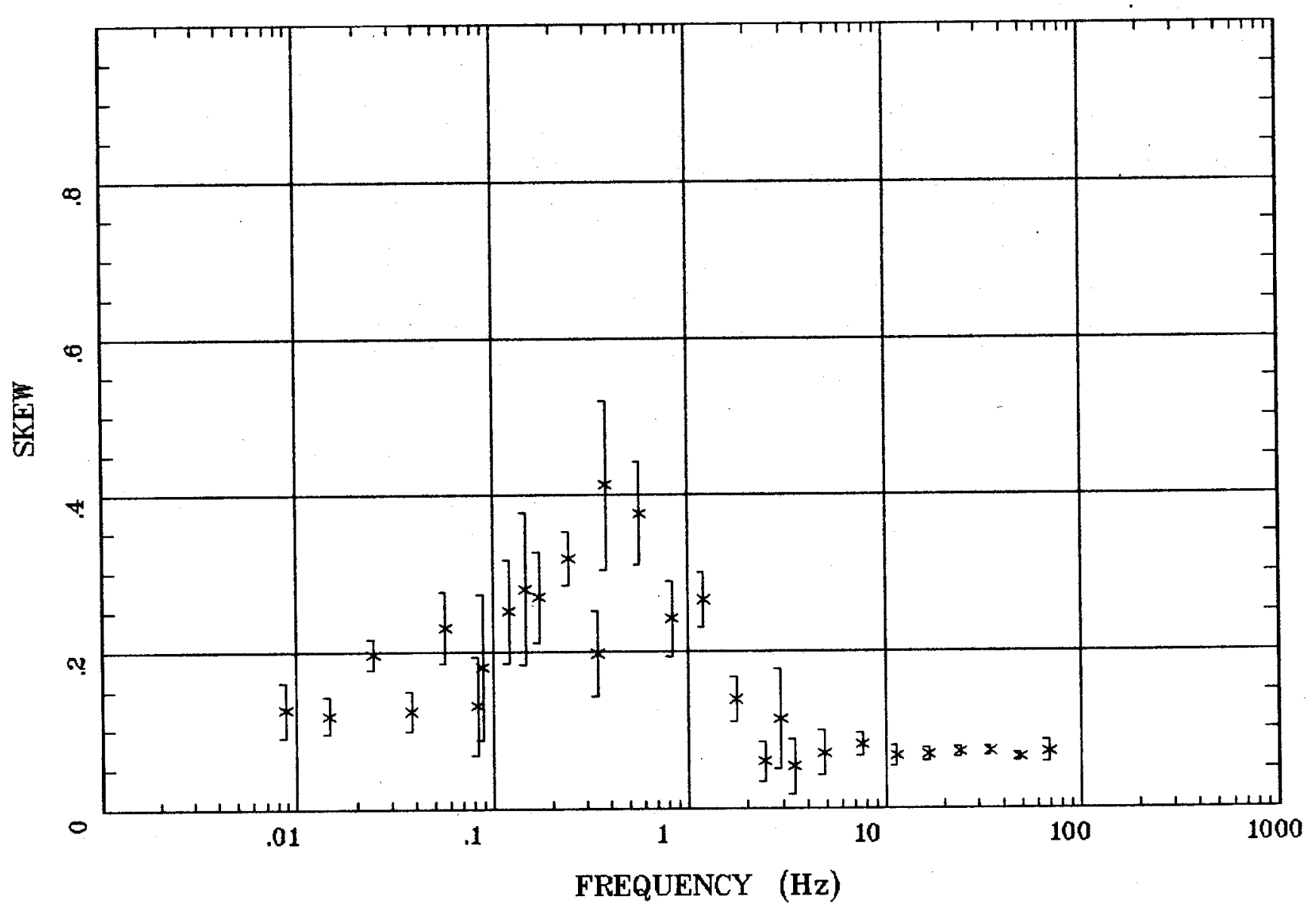

Client:

Remote: none

Acquired: 13:3 Aug 09, 2000 Survey Co:USGS
Rotation:

Filename: hr112b.avg

Channels: Ch1 Ch2 Ch3 Ch4 Ch5 Ch3 Ch4

Plotted: 09:44 Jan 24, 2001

< EMI - ElectroMagnetic Instruments > 
Station 112

E MULT Coh. Ruby Lake NV 100k

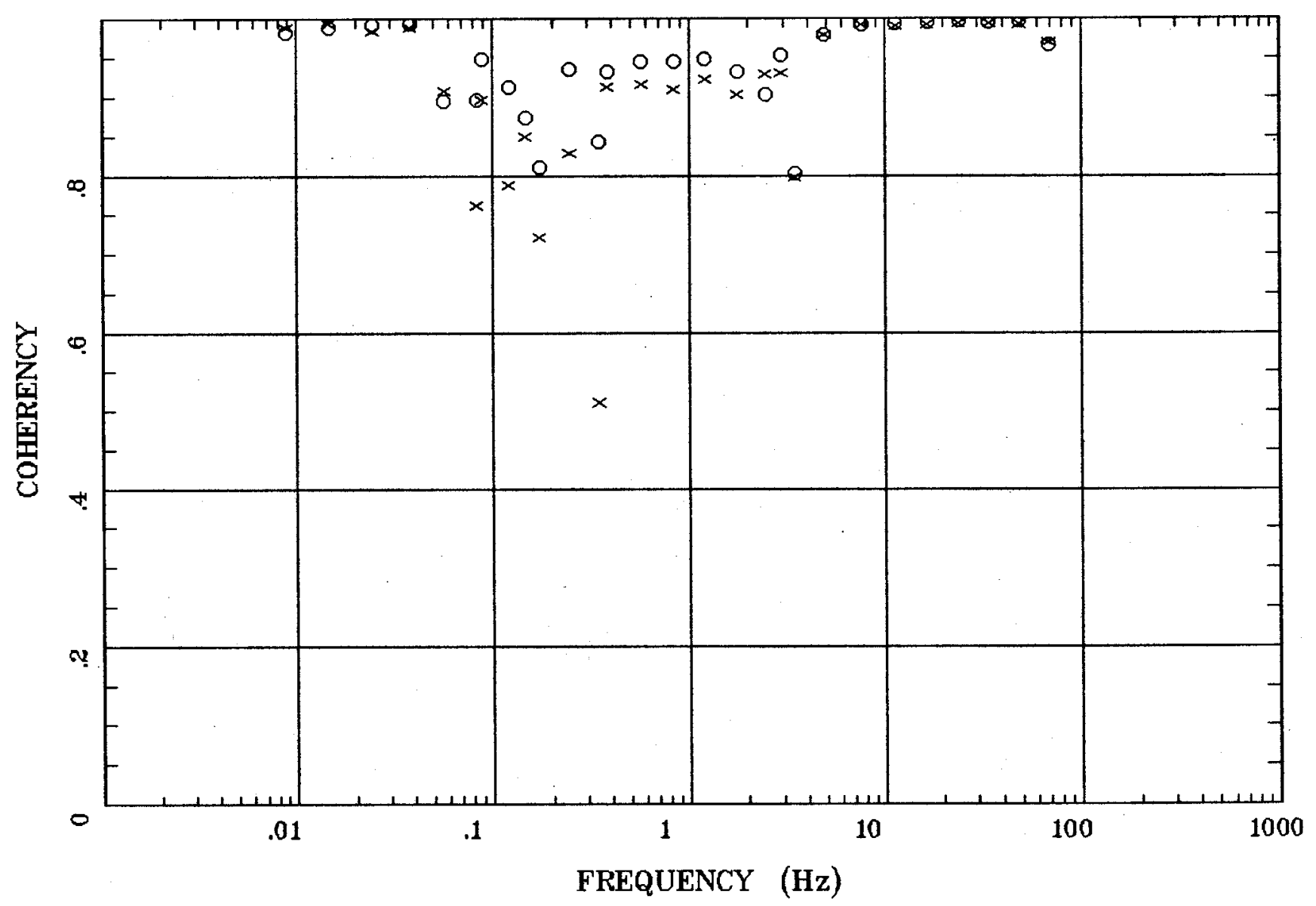

Client:

Remote: none

Acquired: 13:3 Aug 09, 2000

Survey Co:USGS
Rotation:

Filename: hr112b.avg

Channels: Ch1 Ch2 Ch3 Ch4 Ch5 Ch3 Ch4

Plotted: 09:44 Jan 24, 2001

< EMI - ElectroMagnetic Instruments > 
POLAR PLOTS

Ruby Lake NV 100k

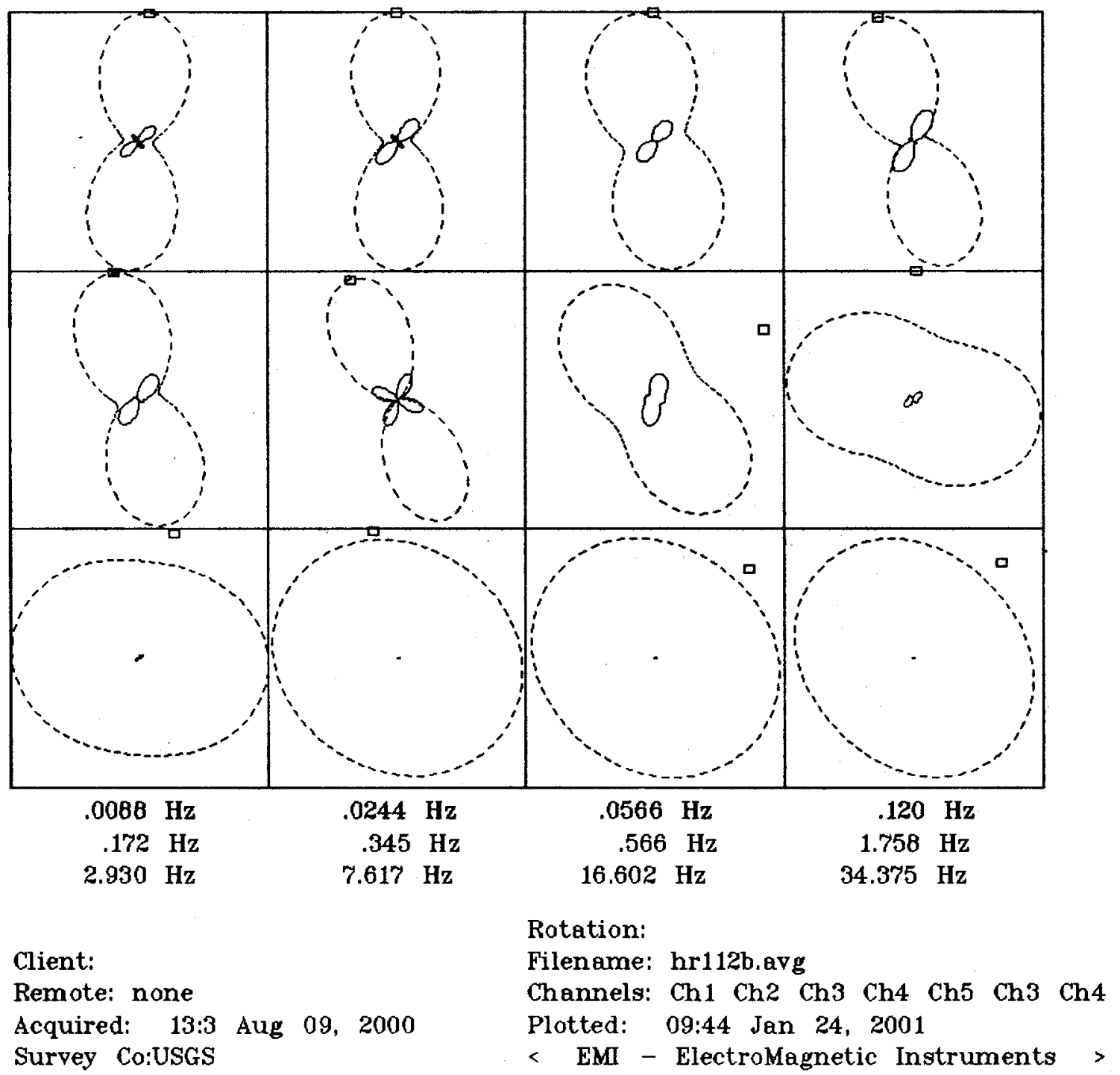


TIPPER MAGNITUDE

Ruby Lake NV 100k

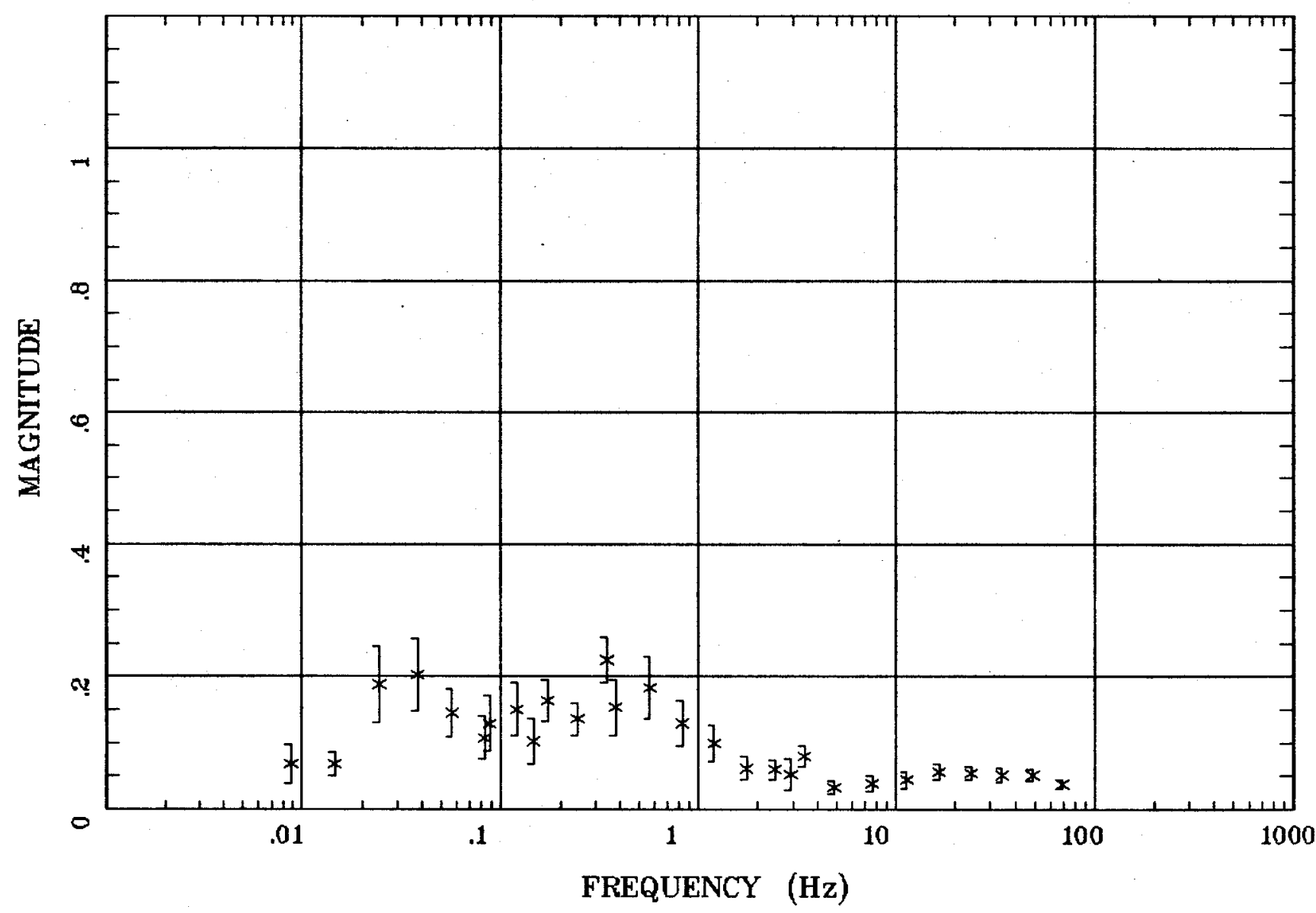

Client:

Remote: none

Acquired: 13:3 Aug 09, 2000

Survey Co:USGS
Rotation:

Filename: hr112b.avg

Channels: Ch1 Ch2 Ch3 Ch4 Ch5 Ch3 Ch4

Plotted: 09:44 Jan 24, 2001

$<$ EMI - ElectroMagnetic Instruments 
TIPPER STRIKE

Ruby Lake NV 100k

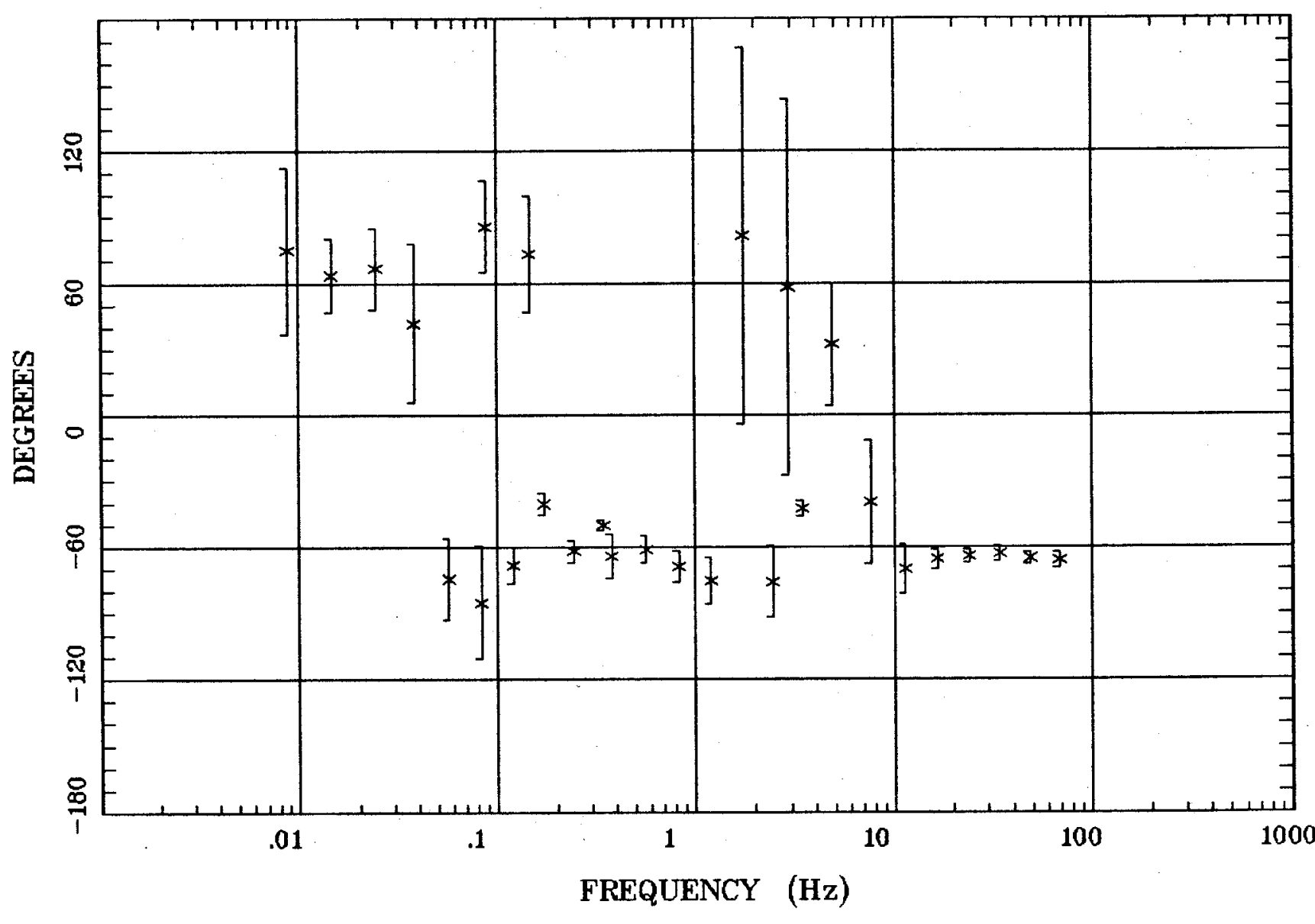

Client:

Remote: none

Acquired: 13:3 Aug 09, 2000

Survey Co:USGS
Rotation:

Filename: hr112b.avg

Channels: Ch1 Ch2 Ch3 ch4 Ch5 Ch3 Ch4

Plotted: 09:44 Jan 24, 2001

$<$ EMI - ElectroMagnetic Instruments 
HzHx.x Coh HzHy.o

Ruby Lake NV 100k

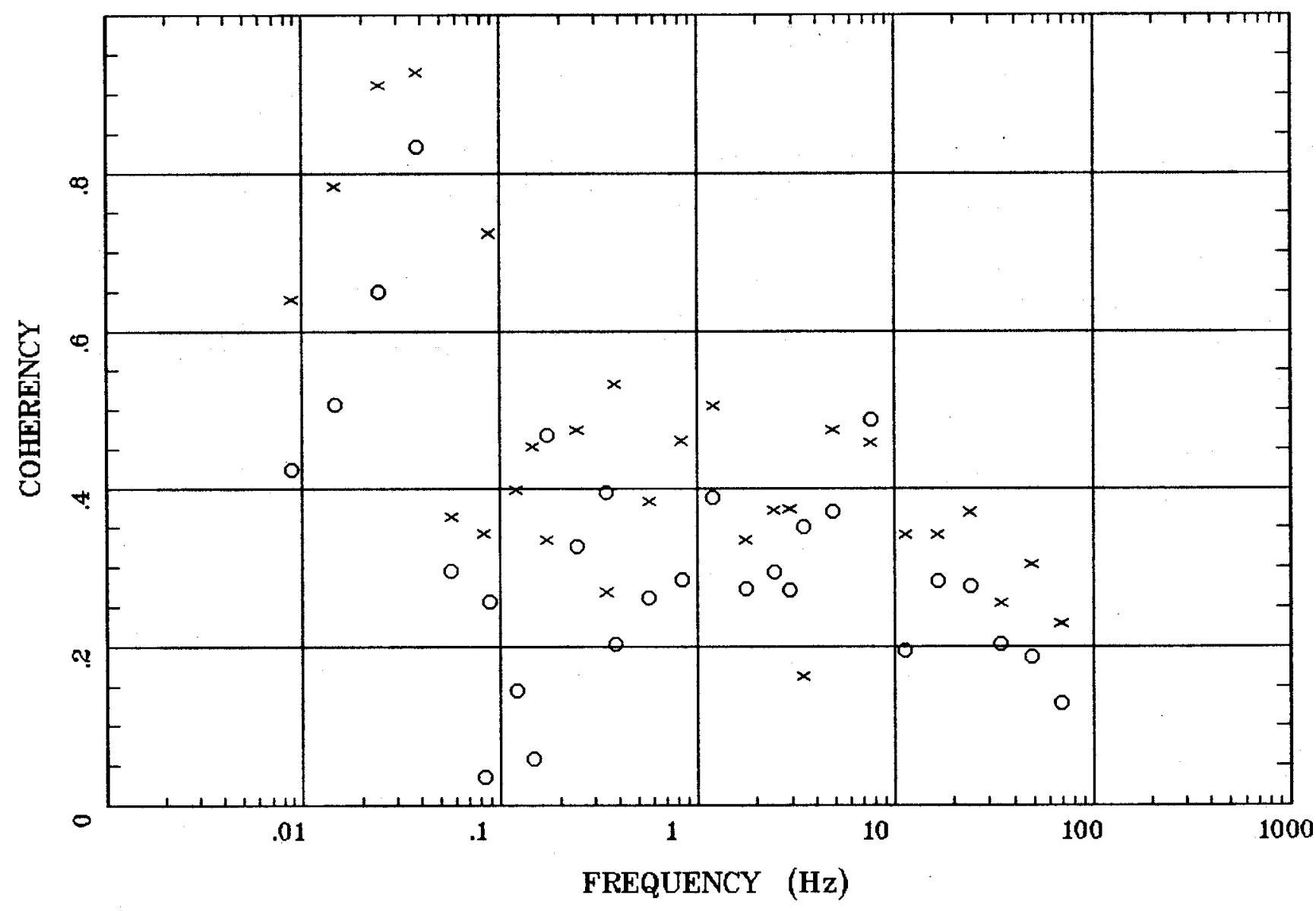

Client:

Remote: none

Acquired: 13:3 Aug 09, 2000 Survey Co:USGS
Rotation:

Filename: hr112b.avg

Channels: Ch1 Ch2 Ch3 ch4 Ch5 Ch3 Ch4

Plotted: 09:44 Jan 24, 2001

< EMI - ElectroMagnetic Instruments 
APPARENT RESISTIVITY

...Pine Valley GettyNest\#1...

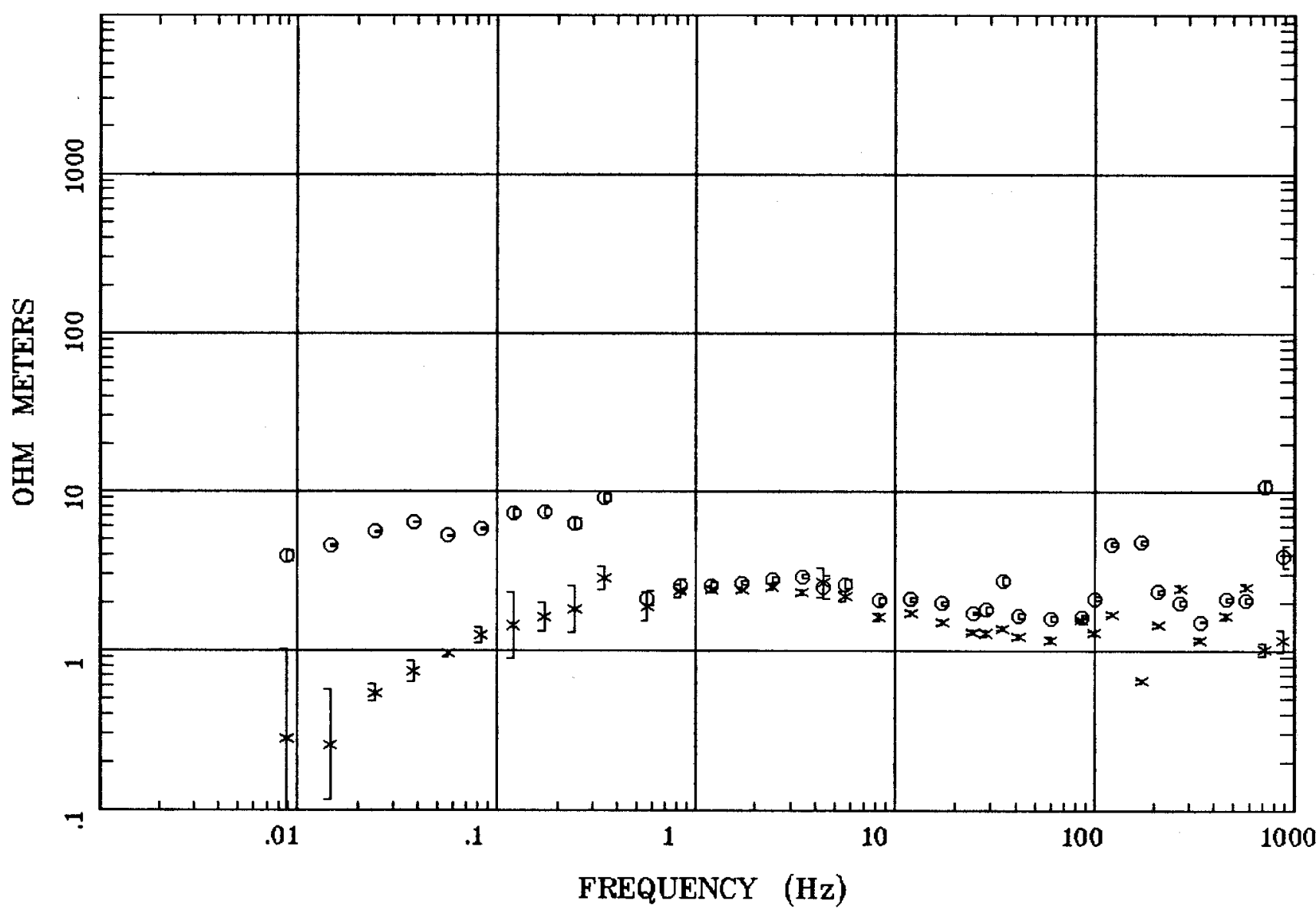

Client:

Remote: $\mathrm{E}$ local ref.

Acquired: 12:5 Jul 24, 1997

Survey Co:
Rotation:

Filename: nn361c.all

Channels: Ch6 Ch7 Ch8 Ch9 Ch10Ch1 Ch2

Plotted: 08:50 Dec 08, 2000

< EMI - ElectroMagnetic Instruments > 
...Pine Valley GettyNest\#1...

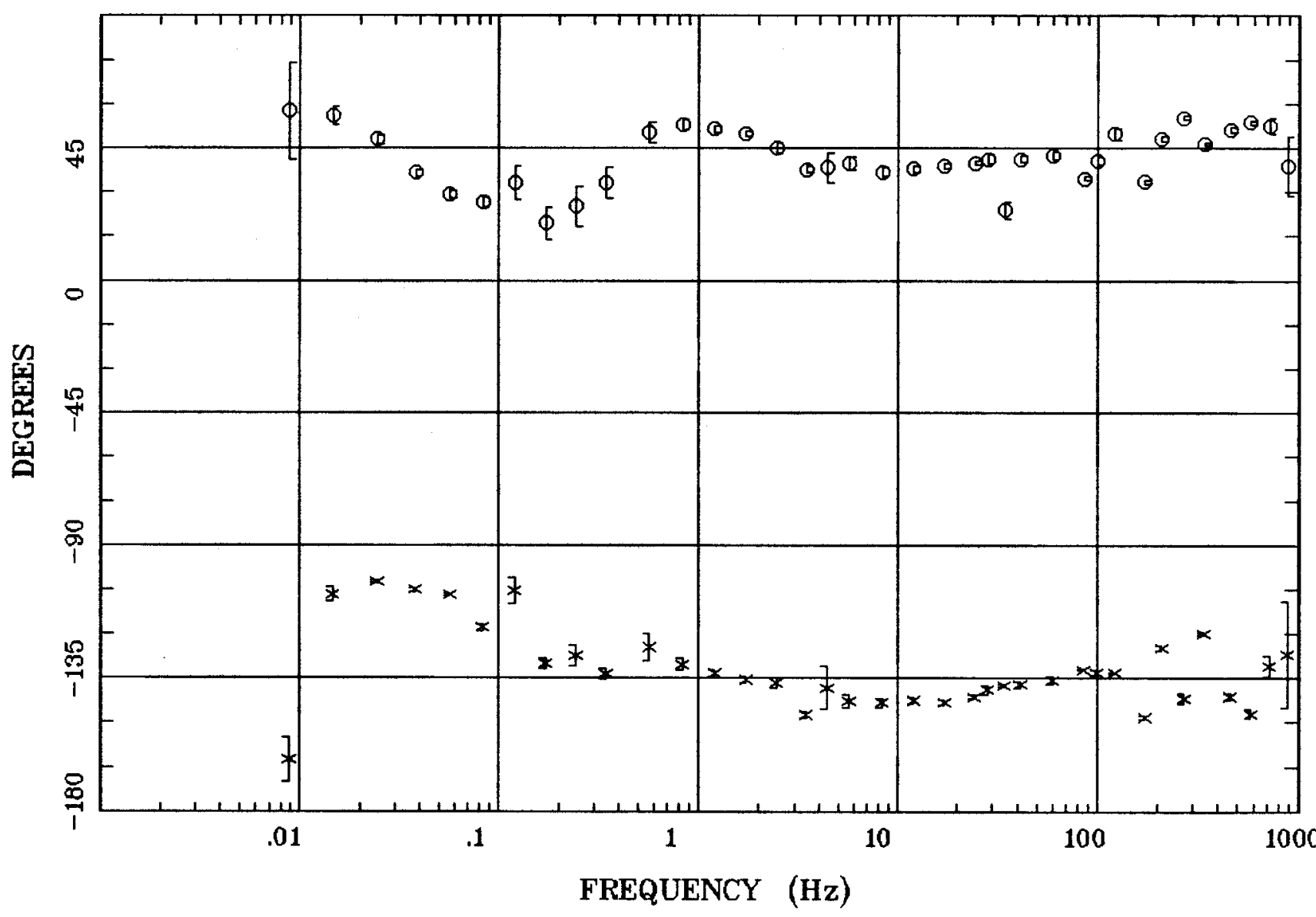

Client:

Remote: E local ref.

Acquired: 12:5 Jul 24, 1997 Survey Co:
Rotation:

Filename: nn361c.all

Channels: Ch6 Ch7 Ch8 Ch9 Ch10Ch1 Ch2 Plotted: 08:50 Dec 08, 2000

< EMI - ElectroMagnetic Instruments 
Station 36

ROTATION ANGLE

...Pine Valley GettyNest\#1...

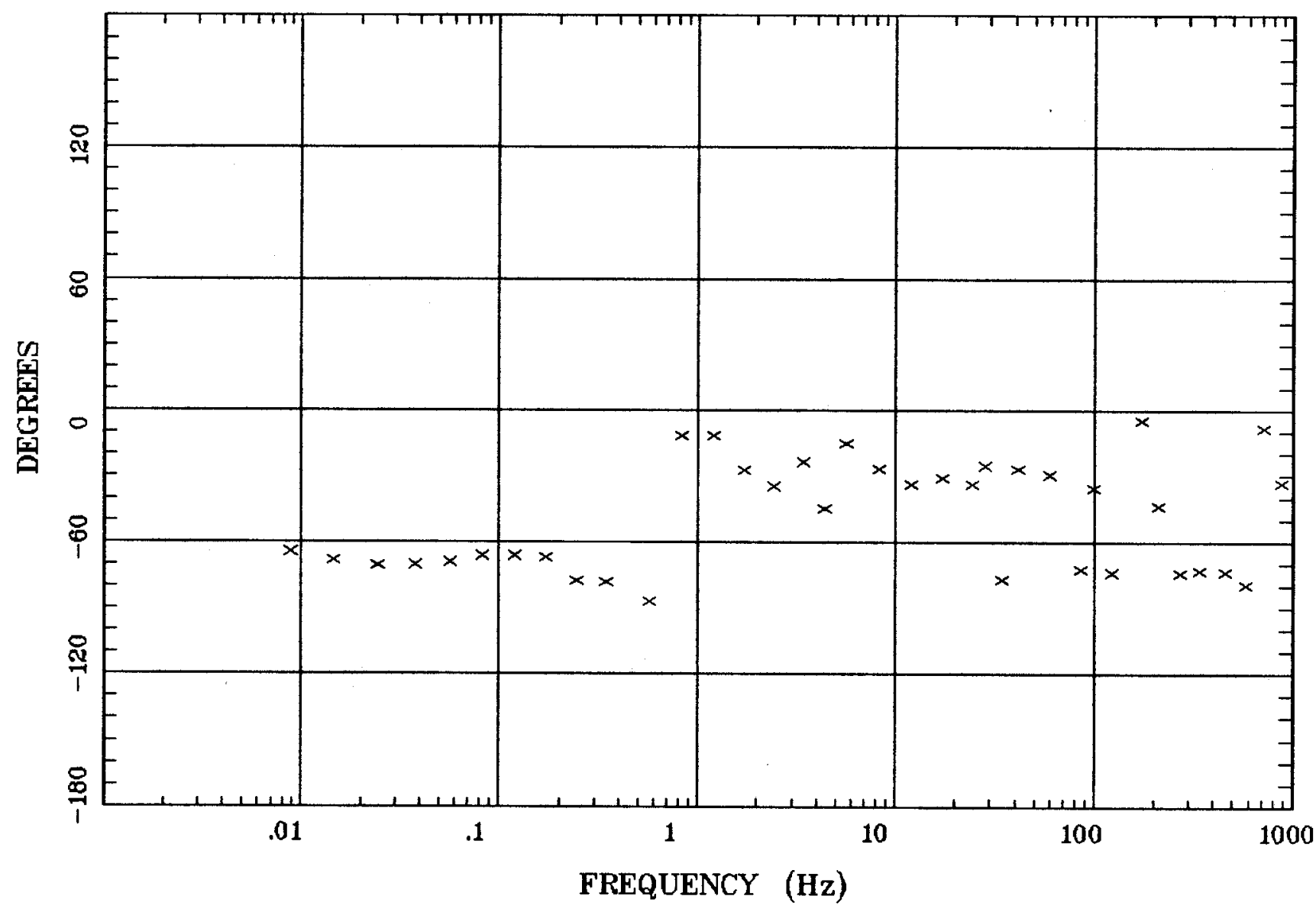

Client:

Rotation:

Remote: E local ref.

Acquired: 12:5 Jul 24, 1997

Filename: nn361c.all

Channels: Ch6 Chy Ch8 Ch9 Ch10ch1 Ch2

Plotted: 08:50 Dec 08, 2000

Survey Co:

< EMI - ElectroMagnetic Instruments 
...Pine Valley GettyNest\#1...

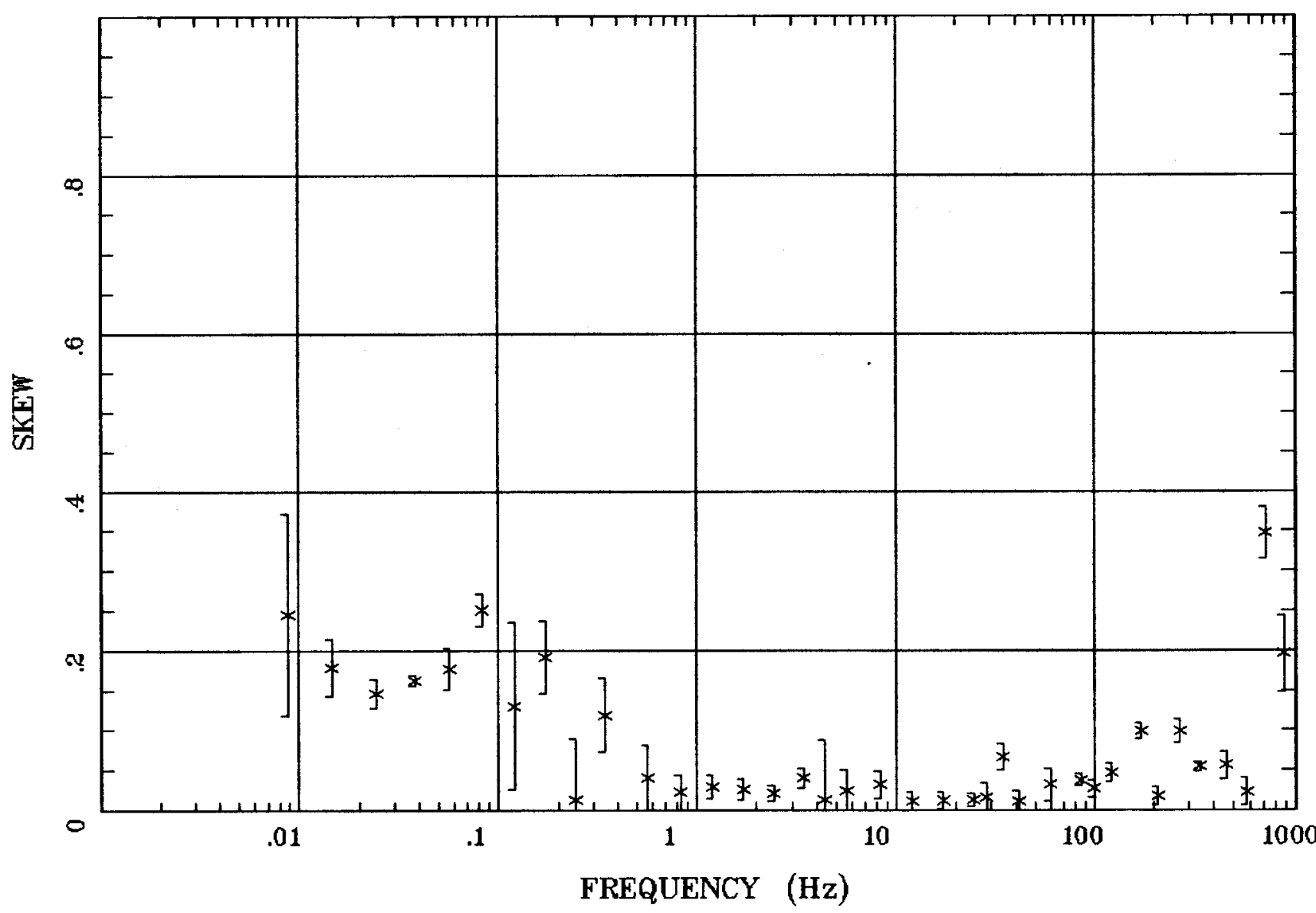

Client:

Remote: E local ref.

Acquired: 12:5 Jul 24, 1997

Survey Co:
Rotation:

Filename: nn361c.all

Channels: Ch6 Ch7 Ch8 Ch9 Ch10Ch1 Ch2

Plotted: 08:50 Dec 08, 2000

< EMI - ElectroMagnetic Instruments 
E MULT Coh.

...Pine Valley GettyNest\#1...

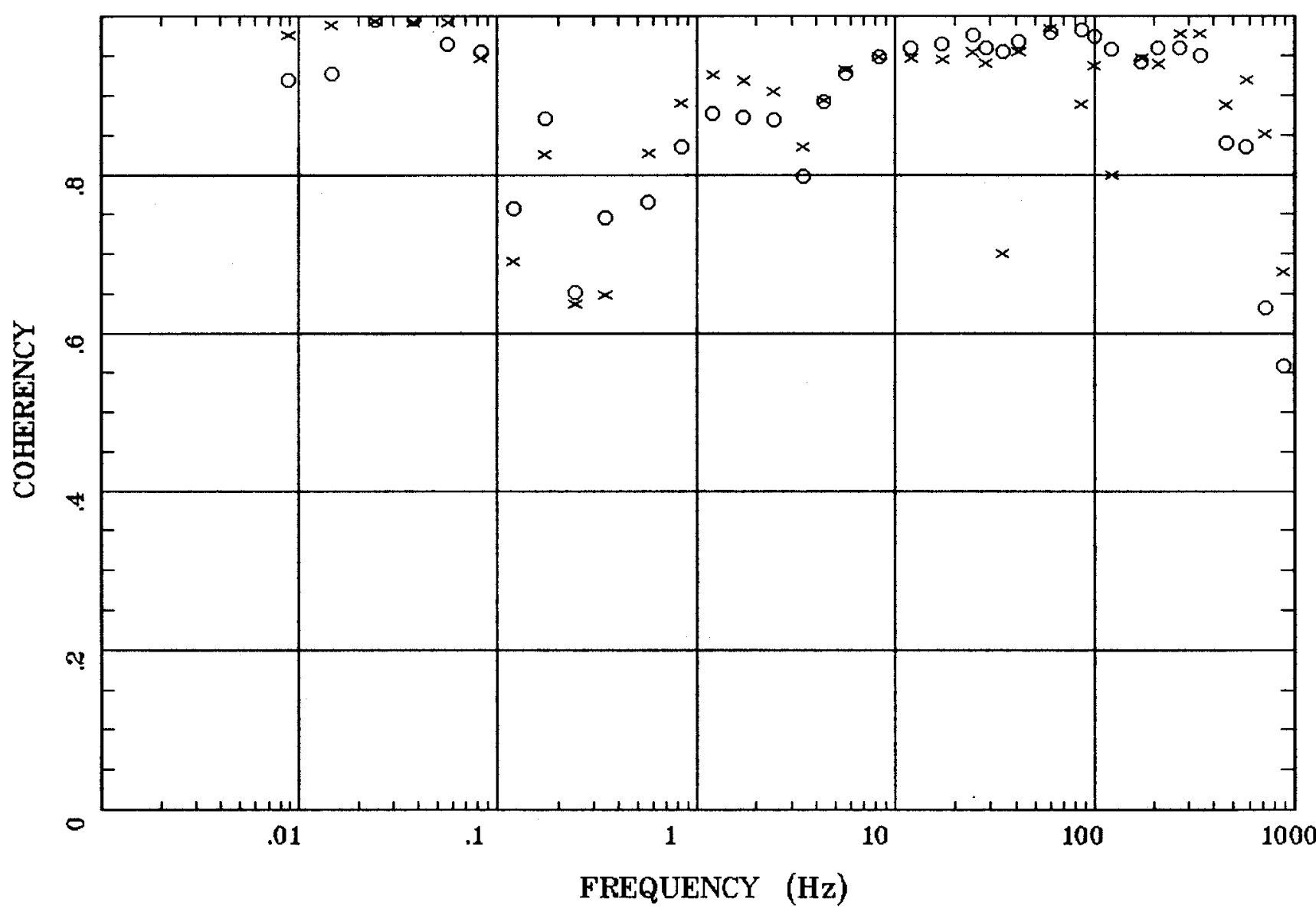

Client:

Remote: E local ref.

Acquired: 12:5 Jul 24, 1997

Survey Co:
Rotation:

Filename: nn361c.all

Channels: Ch6 Ch7 Ch8 Ch9 Ch10Ch1 Ch2

Plotted: 08:50 Dec 08, 2000

< EMI - ElectroMagnetic Instruments 


\section{POLAR PLOTS}

...Pine Valley GettyNest\#1...

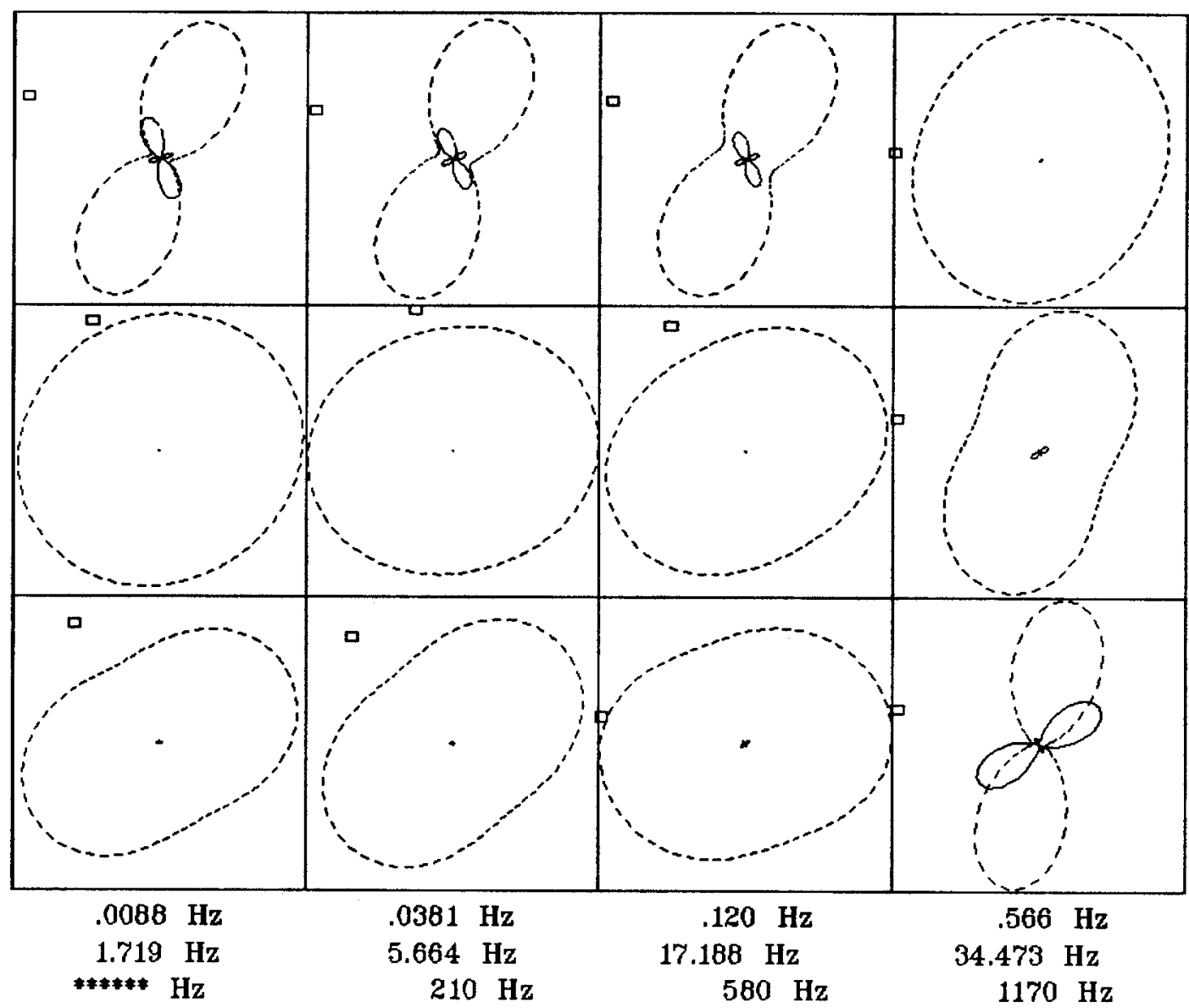

Client:

Remote: E local ref.

Acquired: 12:5 Jul 24, 1997

Survey Co:
Rotation:

Filename: nn361c.all

Channels: Ch6 Ch7 Ch8 Ch9 Ch10Ch1 Ch2

Plotted: 08:50 Dec 08, 2000

$<$ EMI - ElectroMagnetic Instruments > 
TIPPER MAGNITUDE

...Pine Valley GettyNest\#1...

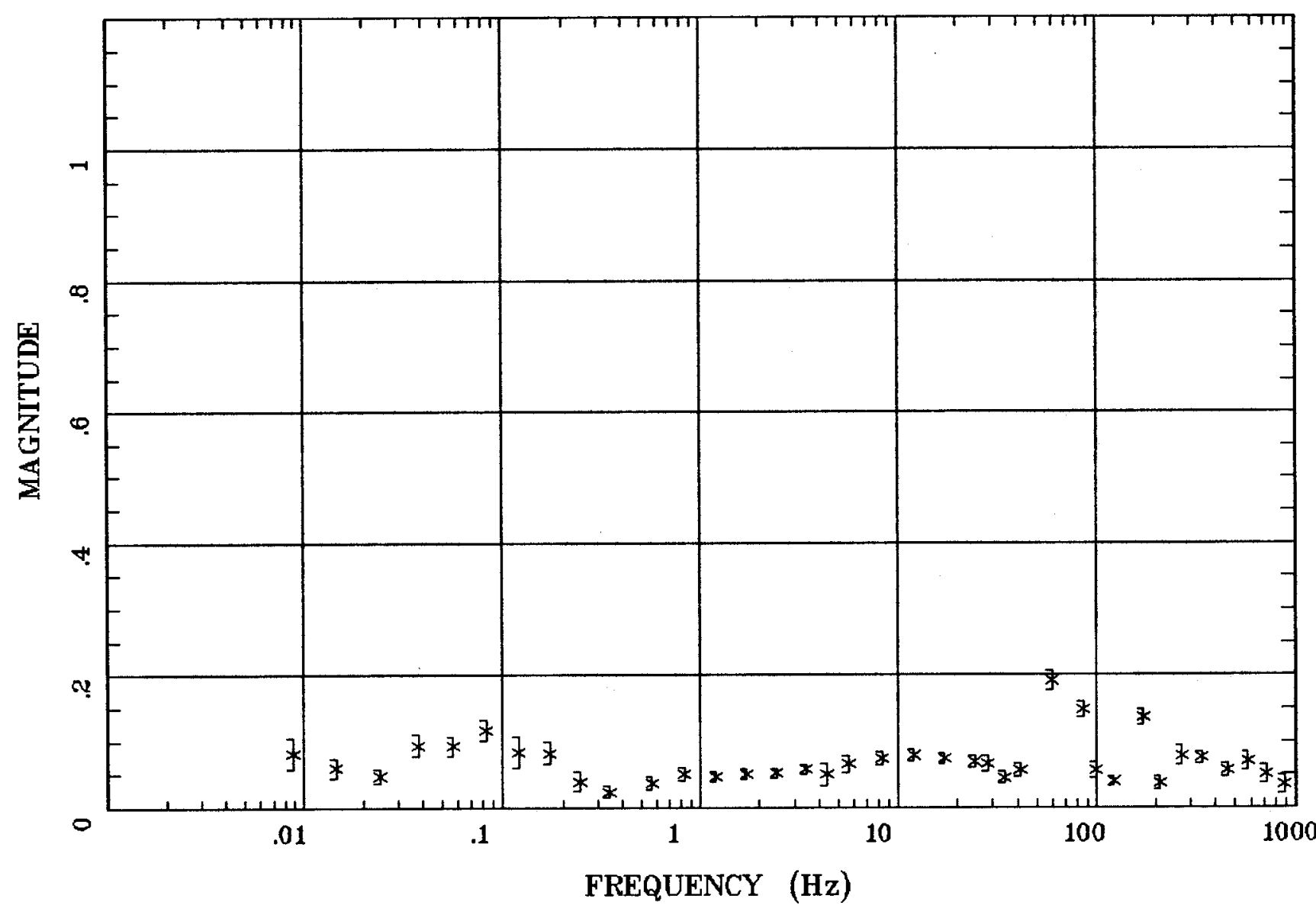

Client:

Remote: E local ref.

Acquired: $12: 5$ Jul 24, 1997 Survey Co:
Rotation:

Filename: nn361c.all

Channels: Ch6 Ch7 Ch8 Ch9 Ch10Ch1 Ch2 Plotted: 08:50 Dec 08, 2000

< EMI - ElectroMagnetic Instruments 


\section{Station 36}

TIPPER STRIKE

...Pine Valley GettyNest $\$ 1 . .$.

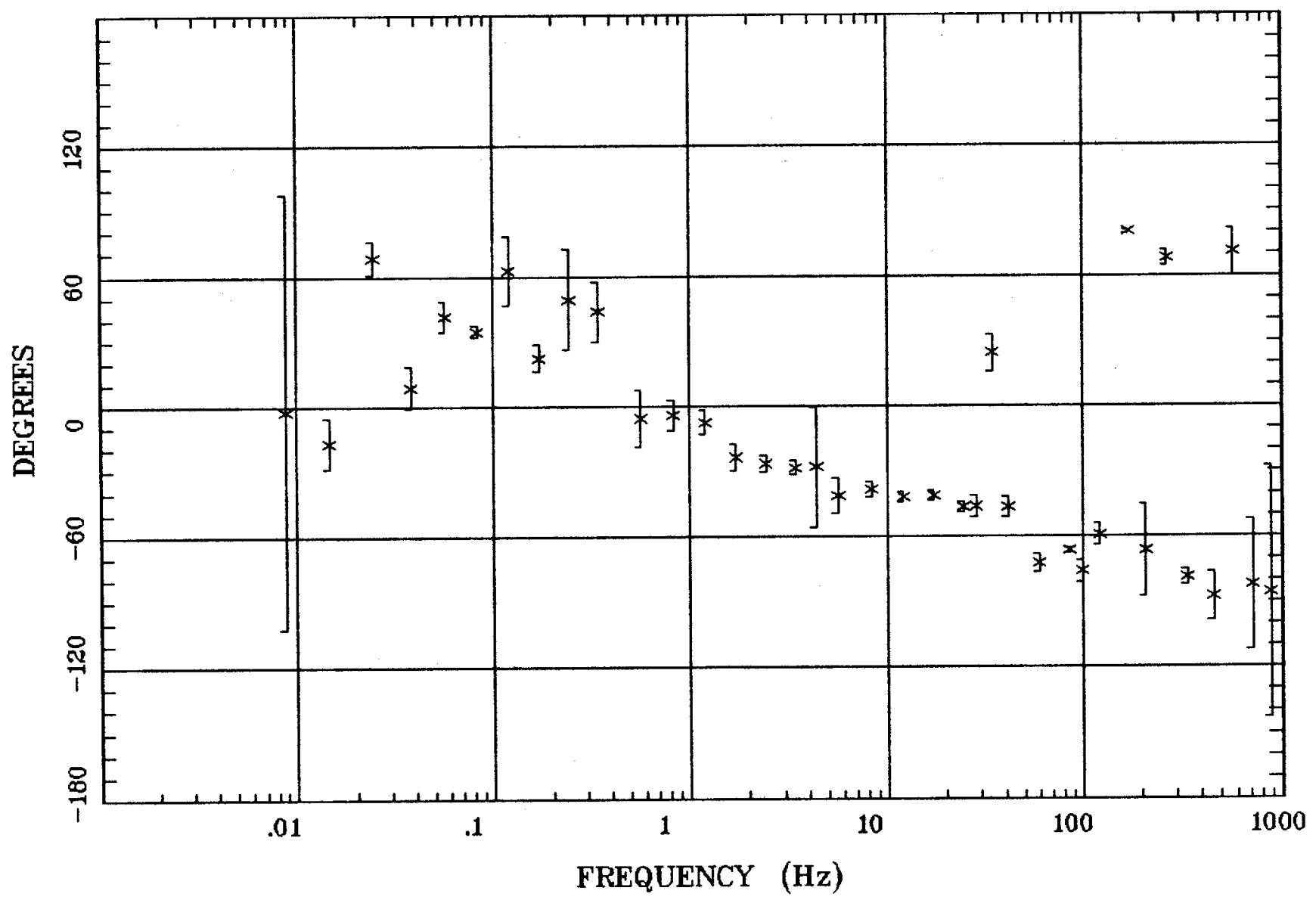

Client:

Rotation:

Remote: E local ref.

Filename: nn361c.all

Acquired: 12:5 Jul 24, 1997

Channels: Ch6 Chr Ch8 Ch9 Ch10Ch1 Ch2

Plotted: 08:50 Dec 08, 2000

Survey Co:

$<$ EMI - ElectroMagnetic Instruments 
HzHx.x Coh HzHy.o

...Pine Valley GettyNest\#1...

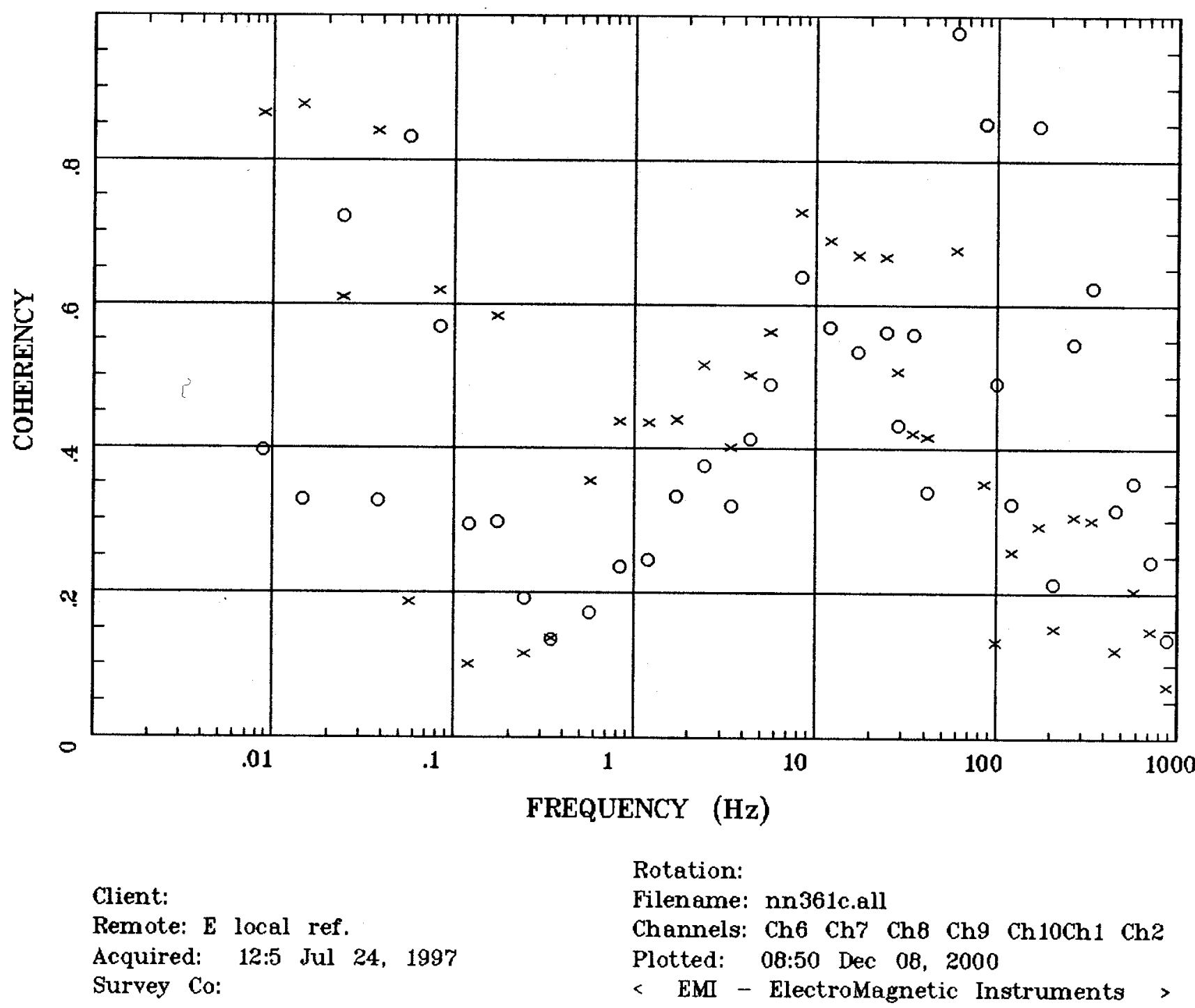

